D $1331 \mathrm{E}$
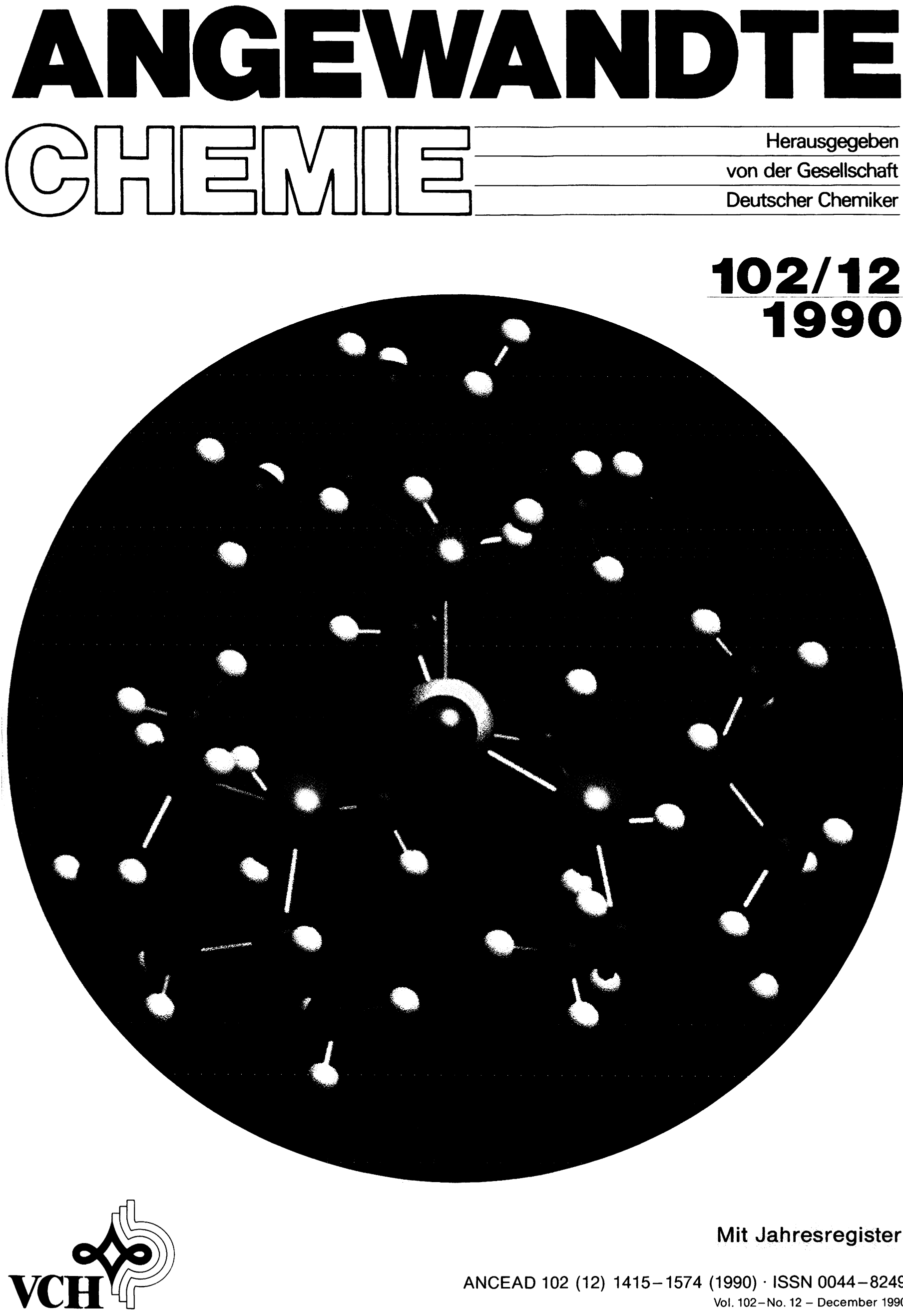

Mit Jahresregister 

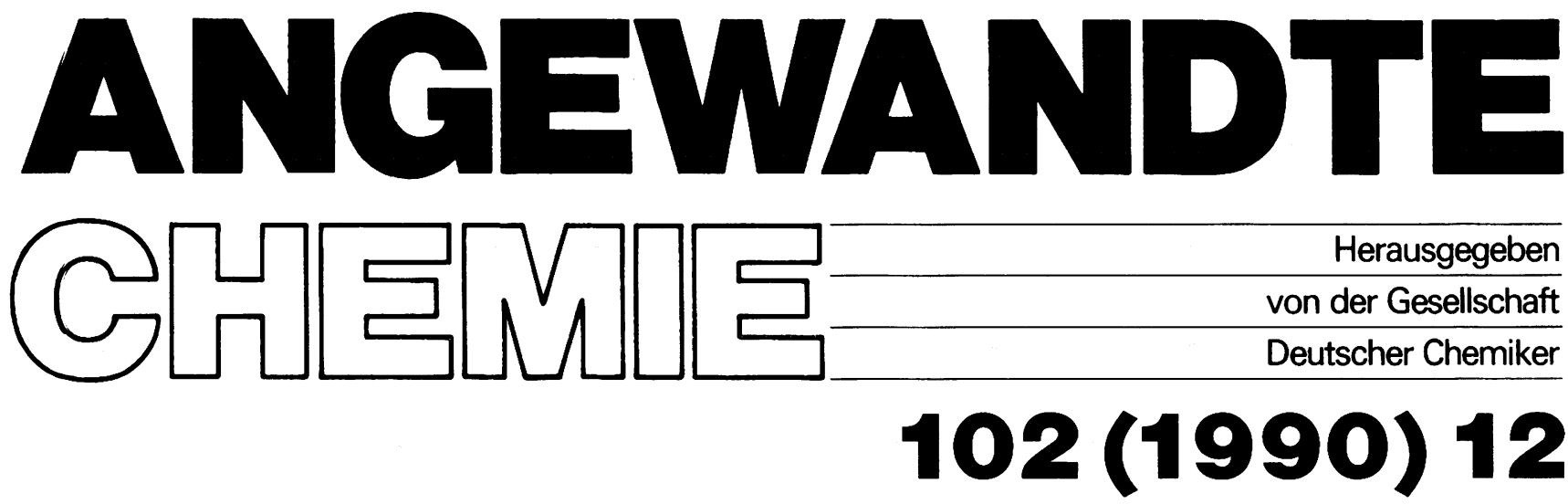

Das Titelbild zeigt die Struktur eines trigonal-bipyramidalen Rhodiumkomplexes, dessen "Schönheit" von einem chiralen $C_{3}$-symmetrischen TriphosphanLiganden herrührt (gelb: Rh, orange: $C O$, violett: $P$, schwarz: $C$, weiß: H). Die verminderte Anzahl asymmetrischer Umgebungen bei Metallkomplexen mit chiralen dreizähnigen $C_{3}$-symmetrischen Liganden sollte bei Reaktionen, die durch derartige Komplexe katalysiert werden, zu hohen Enantioselektivitäten führen. Erste Erfolge konnten bei der Hydrierung von Olefinen erzielt werden. Organische Reaktionen enantioselektiv und katalytisch zu führen, das ist das hochgesteckte Ziel der modernen Organischen Synthese. Erreichen läßt es sich auf dem „Bio-Weg“ (Enzym-, Antikörper-katalysiert) und auf dem „MetallWeg" (Metallkomplex-katalysiert). Einen Beitrag zum Metall-Weg beschreiben M. J. Burk et al. auf S. $1511 \mathrm{ff}$. Die Titelbild-Computergraphik stammt von J. C. Calabrese (DuPont Central Research \& Development).

\section{Aufsatz}

Ein Schlüsselschritt zahlreicher Synthesen in der Organischen und Makromolekularen Chemie, die Addition von Carbenium-Ionen an CC-Doppelbindungen, wird am Beispiel der Lewis-Säure-induzierten Umsetzungen von Alkylchloriden mit Alkenen mechanistisch analysiert. Stereochemische und kinetische Untersuchungen deuten einen wenig verbrückten, Produkt-ähnlichen Übergangszustand an. Reaktivitäts-Selektivitäts-Beziehungen über einen Reaktivitätsbereich von acht Zehnerpotenzen zeigen, daß die Struktur des Übergangszustands nur durch Substituentenvariation in unmittelbarer Nähe des Reaktionszentrums verändert wird.
H. Mayr*

Angew. Chem. 102 (1990) 1415

1428

Knüpfung von CC-Bindungen durch Addition von Carbenium-Ionen an Alkene: Kinetik und Mechanismus 


\section{Zuschriften}

Die Kombination von Porphyrin- und Acetylen-Chemie führt zu interessanten neuen Molekülen. Dies lehrt die Synthese des ersten Acetylen-CumulenPorphyrinoids 1, das als gestrecktes Porphycen betrachtet werden kann.

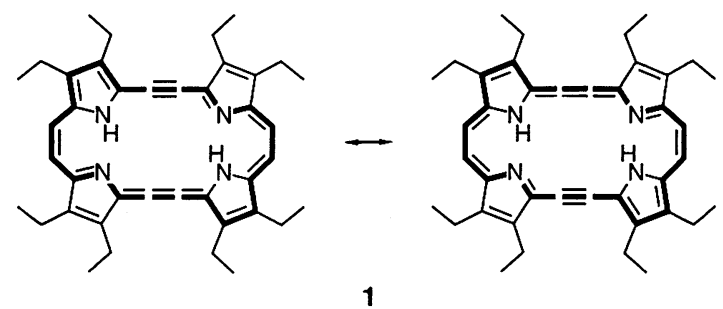

N. Jux, P. Koch, H. Schmickler, J. Lex, E. Vogel*

Angew. Chem. $102(1990) 1429$ 1431
Die enge strukturelle Verflechtung von Porphyrin- und Porphycen-System wird mit dem [22]Porphyrin(2.2.2.2) $\mathbf{1 / 2}$ besonders augenfällig demonstriert: [22]Porphyrin(2.2.2.2) existiert aus Spannungsgründen nicht als das Isomer mit Porphyrin-Symmetrie $\left(D_{2 h}\right)$ 1, sondern liegt als gestrecktes Porphycen 2 vor.

1

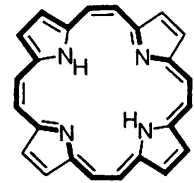

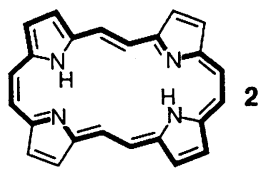

E. Vogel*, N. Jux, E. Rodriguez-Val,

J. Lex, H. Schmickler

Angew. Chem. 102 (1990) 1431

1434

Die Porphyrin-Homologen: [22]Porphyrin(2.2.2.2), ein ,,gestrecktes Porphycen“
Die Ringkontraktion eines Porphycenderivats ergibt ein Isocorrolderivat, dessen Ringgerüst deutlich stärker als das von Corrol von der Planarität abweicht. Nach spektroskopischen Befunden hat man es dennoch mit einer aromatischen Verbindung zu tun. 1 und $\mathbf{3}$ sind die Stammverbindungen Corrol bzw. Isocorrol.

1
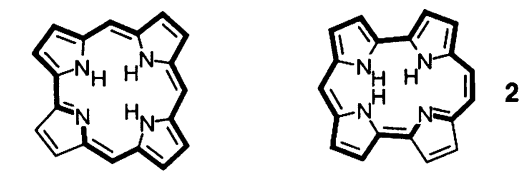

Hans Fischers Octaethylporphyrin (OEP) ist bis heute von allen synthetischen Porphyrinen das bestverfügbare und meistuntersuchte. Durch eine stufenarme Synthese mit hohen Ausbeuten konnte nun das bisvinyloge OEP 1 gewonnen werden. Dieses ist durch chemische Stabilität, gute Zugänglichkeit und die bisher bei einem organischen Farbstoff intensivste UV/VIS-Absorption ein System mit vielseitigen Perspektiven.<smiles></smiles>

H. König, C. Eickmeier, M. Möller, U. Rodewald, B. Franck*

Angew. Chem. 102 (1990) 1437 1439

Synthese eines bisvinylogen Octaethylporphyrins
Hämatoporphyrin hat Bedeutung für eine photodynamische Tumortherapie (PDT). Funktionelle Nachteile der ,oberen“ Molekülhälfte dieses Photosensibilisators, wie chemische Instabilität und Stereoisomerie, stehen der medizinischen Anwendung jedoch noch im Wege. Das neue, aus zwei ,unteren“" Molekülhälften aufgebaute [22]Coproporphyrin II 1 ist durch Stabilität, sterische Einheitlichkeit und längerwellige, intensive UV/VIS-Banden charakterisiert. 1 erwies sich als aromatisches [22]Annulen.
S. Will, A. Rahbar, H. Schmickler, J. Lex, E. Vogel*

Angew. Chem. 102 (1990) 1434.

Isocorrole: Neuartige tetrapyrrolische Makrocyclen

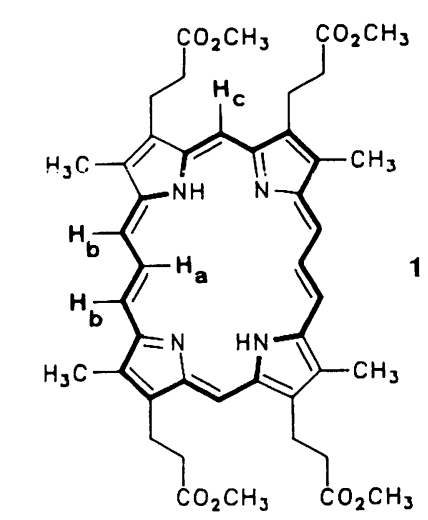

S. Beckmann, T. Wessel, B. Franck *, W. Hönle, H. Borrmann, H. G. von Schnering

Angew. Chem. 102 (1990) 1439. $144 i$

[22]Coproporphyrin II für die photodynamische Therapie 
Nur 0.0022 Moläquivalente des Katalysators $\left[\mathrm{Co}_{2}(\mathrm{CO})_{8}\right]$ werden benötigt, um mit einer Wechselzahl von etwa 220 das Cyclopentenon 1 aus 1-Heptin, Ethylen und CO herzustellen. Diese erste katalytische PausonKhand-Reaktion (PKR) mit einem nichtgespannten Alken liefert 1, eine Vorstufe des für die Parfümindustrie wichtigen Methyl-transdihydrojasmonats, in etwa $50 \%$ Ausbeute. Auch die wichtigsten Nebenprodukte einer PKR wurden hier identifiziert und mögliche Wege ihrer Bildung vorgeschlagen.

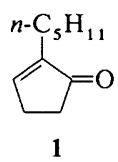

V. Rautenstrauch*, P. Mégard, J. Conesa, W. Küster

Angew. Chem. 102 (1990) 1441

2-Pentylcyclopent-2-en-1-on durch katalytische Pauson-Khand-Reaktion
Das leicht zugängliche Tetrabrom|2.2|paracyclophan läßt sich mit Kalium-tert-butylalkoholat in Tetrahydrofuran schrittweise vierfach dehydrobromieren. Die dabei gebildeten [2.2]Paracyclophanine werden von Furan, Diphenylisobenzofuran und Tetraphenylcyclopentadienon $\mathrm{zu}$ entsprechenden $\mathrm{Cy}$ cloaddukten effizient abgefangen. Mit besonders hoher Ausbeute $(69 \%)$ wird das Octaphenydibenzo[2.2]paracyclophan-1,9-dien 1 isoliert, das formal aus zwei Hexaphenylbenzol-Einheiten besteht.

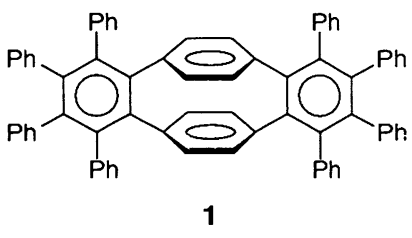

A. de Meijere*, J. Heinze, K. Meerholz, O. Reiser, B. König

Angew. Chem. 102 (1990) 1443.

Ein Syntheseäquivalent für [2.2]Paracyclophan-1,9-diin: Octaphenyl-1:2,9:10dibenzo[2.2]paracyclophan-1,9-dien und seine Reduktion zum Hexaanion

Tandem-Mannich-Michael-Reaktionen und Aza-Diels-Alder-Reaktionen gehen die Aminosäureester-Imine 1 in Gegenwart unterschiedlicher Lewis-Säuren mit dem Danishefsky-Dien 2 bzw. dem Brassard-Dien 3 ein. Die Produkte entstehen mit guten Ausbeuten und hohen Diastereomerenüberschüssen. Durch Abspaltung der chiralen Hilfsgruppen werden daraus ungesättigte Lactame wie 4 und Enaminone wie $\mathbf{5}$ erhalten, die als Synthesebausteine Verwendung finden.<smiles>[R7]CC(C)C(N=C([R])C)C(=O)O</smiles>

1<smiles>C=C(C=COC)OC</smiles>

2<smiles>C=C(C=C(OC)OC)OC</smiles>

3<smiles>CCC1CC(O)=CC(=O)N1</smiles>

4<smiles>[R]C1CC(=O)C=CN1</smiles>

5
H. Waldmann*, M. Braun, M. Dräger

Angew. Chem. 102 (1990) 1445. .. 1447

Aminosäureester als chirale Hilfsgruppen in Lewis-Säure-katalysierten Umsetzungen elektronenreicher Siloxydiene mit Iminen
Durch einen hohen Anteil an Baufehlern, z. B. durch wechselnde Schichtabfolgen, sind die Realstrukturen der $(\mathrm{BiO})_{2} \mathrm{Sr}_{2} \mathrm{Ca}_{n-1} \mathrm{Cu}_{n} \mathrm{O}_{2 n+2}$-Supraleiter gekennzeichnet, wie hochaufgelöste transmissionselektronenmikroskopische Aufnahmen zeigen. Art und Umfang der Defekte können sich bei den einzelnen Ansätzen erheblich unterscheiden, so daß die Schwierigkeit, reproduzierbare Probeneigenschaften zu erhalten, verständlich wird.

\section{J.-H. Müller, M. Schwarz, R. Gruehn*}

Angew. Chem. 102 (1990) 1448.

.1450

Darstellung und transmissionselektronenmikroskopische Untersuchung von $\mathrm{Bi}(\mathrm{Pb})-\mathrm{Sr}-\mathrm{Ca}-\mathrm{Cu}-\mathrm{O}-\mathrm{Supraleitern}$
For the USA and Canada: ANGEWANDTE CHEMIE (ISSN 0044-8249) is published monthly by VCH Publishers, Inc., 303 N.W. 12th Avenue, Deerfield Beach FL 33442-1788; Telefax (305) 428-8201; Telephone (305) 428-5566 or (800) 422-8824. Second-class postage paid at Deerfield Beach FL 33441. Annual subscription price: US\$ 440.00/467.00 (air mail) including postage and handling charges. 1991 US\$ 535.00/ 560.00 (air mail). Rate for individuals whose institution already subscribes, who are retired or self-employed consultants: $\$ 165.00 / 190.00$ (air mail). - Printed in the Federal Republic of Germany.

U.S. POSTMASTER: Send address changes to ANGEWANDTE CHEMIE, c/o VCH Publishers, Inc., 303 N.W. 12th Avenue, Deerfield Beach FL 33442-1788.

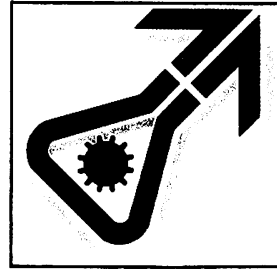


Ausgehend von 5-Cyclodecinol lassen sich nach einem Baukastenprinzip metallstabilisierte, vierfach überbrückte Cyclobutadienophane wie 1-4 aufbauen.

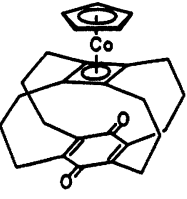

1

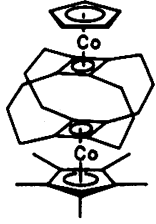

2

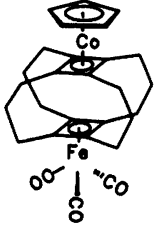

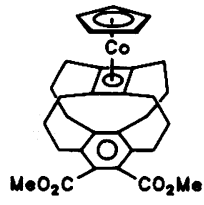

4
R. Gleiter*, V. Schehlmann

Angew. Chem. 102 (1990) 1450.

1452

Aufbau metallstabilisierter, vierfach überbrückter Cyclobutadienophane
Das hochgespannte Dewar-Benzol 1 sowie das Prisman 2 lassen sich in wenigen Stufen aus Cyclooctin erhalten. 2 entsteht durch Bestrahlung von 1 mit langwelligem Licht; bei $\lambda=250 \mathrm{~nm}$ entstehen nur Polymere, vermutlich über das entsprechende [5]Paracyclophan.

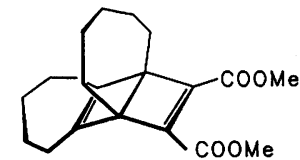

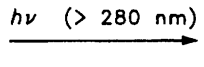

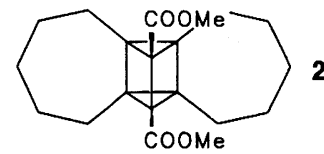

R. Gleiter*, B. Treptow

Angew. Chem. 102 (1990) 1452 1454

Doppelt überbrückte Prisman-, DewarBenzol- und Benzol-Derivate aus Cyclooctin und 1,8-Cyclotetradecadiin: en route zu Propella[ $\left.\mathrm{n}_{3}\right]$ prismanen
Eine spontane Reduktion von $\mathrm{Cu}^{\mathrm{II}} \mathrm{zu} \mathrm{Cu}^{\mathrm{I}}$ findet statt, wenn $\mathrm{CuBr}_{2}$ mit dem dreizähnigen $\mathrm{SN}_{2}$-Liganden ddtp in siedendem Ethanol/ Aceton umgesetzt wird. Der entstehende $\mathrm{Cu}_{4}$-Titelkomplex enthält Thioetherbrücken mit großen $\mathrm{Cu}-\mathrm{S}-\mathrm{Abständen}$ und $\mathrm{Cu}-\mathrm{S}-\mathrm{Cu}$ Winkeln (160.6 $6^{\circ}$ ). Die $\mathrm{Cu}_{2} \mathrm{Cu}_{2}$-Rechteckstruktur ist für $\mathrm{Cu}^{1}$-Komplexe präzedenzlos. $\left[\mathrm{Cu}_{4}(\mathrm{ddtp})_{2} \mathrm{Br}_{4}\right]$ kann als Referenzverbindung für EXAFS-Untersuchungen an blauen Kupferproteinen dienen.<smiles>Cc1cc(C)n(CCSCCn2nc(C)cc2C)n1</smiles>

ddtp
A. L. E. Stoffels, W. G. Haanstra, W. L. Driessen*, J. Reedijk

Angew. Chem. 102 (1990) 1454. 1455

$\left[\mathrm{Cu}_{4}(\mathrm{ddtp})_{2} \mathrm{Br}_{4}\right]$, ein ungewöhnlicher, vierkerniger $\mathrm{Cu}^{1}$-Komplex mit verbrükkenden Thioether- und Bromidliganden
Je nach Elektrophil unter Retention oder Inversion der Konfiguration erfolgt die Substitution der in Lösung konfigurationsstabilen chiralen Benzyllithium-Verbindung 2, die durch Deprotonierung des entsprechenden optisch aktiven Benzylcarbamates 1 generiert wurde. Die Produkte 3 sind als Synthesebausteine von Interesse. $\mathrm{Cb}=\mathrm{C}(=\mathrm{O}) \mathrm{N} i P r_{2}$.

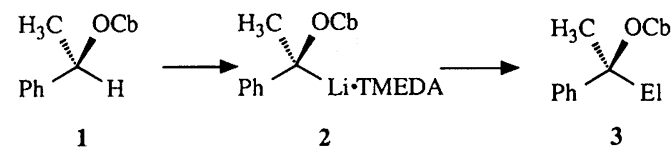

D. Hoppe*, A. Carstens, T. Krämer

Angew. Chem. 102 (1990) 1455 . .1456

Erzeugung eines konfigurationsstabilen, chiralen Benzyllithium-Derivates und kapriziöse Stereochemie seiner elektrophilen Substitution
Eine allgemeine, sehr einfache präparative Lösung für chirale Synthone des Typs 3 bieten die Lithiumkomplexe 1. Sie werden durch asymmetrische Deprotonierung der entsprechenden prochiralen ,nichtaktivierten“ Alkylcarbamate mit sec-Butyllithium/(-)-Spartein erzeugt. 1 läßt sich durch Elektrophile stereospezifisch zu den geschützten Hydroxy-Derivaten 2 ( $>95 e e$ ) substituieren, und die $C b x$-Gruppe ist leicht abspaltbar. $\mathrm{R}^{1}=\mathrm{Alkyl}, \mathrm{Cbx}=$ Carbamoylrest von 1.

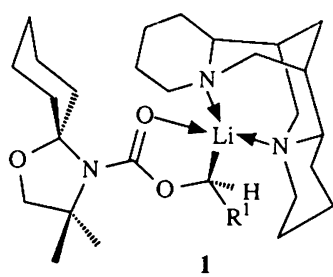<smiles>[R]C([B])OC(=O)[CH]C</smiles><smiles>[R]C(O)C(=O)C=O</smiles>

\section{Hoppe*, F. Hintze, P. Tebben}

Angew. Chem. 102 (1990) 1457 1459

Chirale Lithium-1-oxyalkanide durch asymmetrische Deprotonierung; enantioselektive Synthese von 2-Hydroxyalkansäuren und sekundären Alkanolen 
Die Hydrodimerisierung von trans- und von cis-1-Deuterio-1-hexen mit dem chiralen Präkatalysator $r a c$-[Ethylenbis(tetrahydroindenyl)]zirconiumdichlorid zu den threo- und erythro-Isomeren von 6-Deuterio-5-deuteriomethylundecan ergab Diastereomerenverhältnisse von ca. $2.4: 1$, die sich je nach $(E)$ - oder $(Z)$ Konfiguration des Olefins umkehren. Die Befunde bestätigen Beobachtungen von Pino et al., daß $\alpha$-Olefine sich in $\mathrm{Zr}-\mathrm{H}$ - und $\mathrm{Zr}$-Alkyl-Bindungen mit entgegengesetzter enantiofacialer Orientierung einschieben.
H. Krauledat, H.-H. Brintzinger*

Angew. Chem. 102 (1990) 1459 1460

Isotopeneffekte bei der $\alpha$-Olefineinschiebung in Zirconocen-Polymerisationskatalysatoren: Hinweise auf einen Übergangszustand mit $\alpha$-agostischer Wechselwirkung
Nicht nur das bisher einfachste Alkylosmiumoxid, $\mathrm{Me}_{4} \mathrm{OsO}$, das in der Gasphase stabil ist, und dessen Struktur (Bild rechts) durch Elektronenbeugungsanalyse aufgeklärt werden konnte, sondern auch das thermolabile $\mathrm{Et}_{4} \mathrm{OsO}$ und ein gemischt alkyliertes Derivat ließen sich in guten Ausbeuten herstellen. Ferner wird über das strukturell neuartige Trimer $\left[\left\{(\mathrm{py}) \mathrm{OsO}_{2} \mathrm{Me}_{2}\right\}_{3}\right]$ mit planarem $\mathrm{Os}_{3} \mathrm{O}_{3}$-Sechsring berichtet.

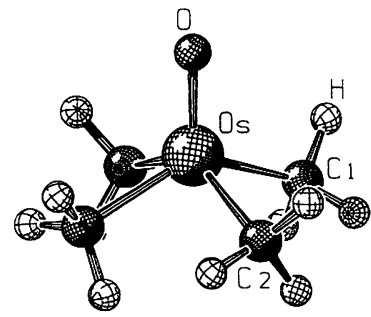

W. A. Herrmann*, S. J. Eder, P. Kiprof, K. Rypdal, P. Watzlowik

Angew. Chem. 102 (1990) 1460

Organoosmiumoxide - gezielte Synthesen und Strukturen
Alle zehn Sauerstoffatome des acyclischen Oligoethers $1 \mathrm{c}$ beteiligen sich an der Koordination von $\mathrm{Ba}^{2 \oplus} \mathrm{im}$,Skorpion"-Komplex $1 \mathrm{c} \cdot \mathrm{Ba}(\mathrm{SCN})_{2} \cdot \mathrm{Me}_{2} \mathrm{CO}$. Die elfte Koordinationsstelle besetzt das Acetonmolekül. Ein weiteres Kennzeichen des Komplexes sind starke $\mathrm{O}-\mathrm{H} \cdots \mathrm{N}$-Wasserstoffbrücken zu den Thiocyanat-Gegenionen. Die Liganden 1 zeigen zudem eine Größenselektivität für Kationen mit Ionenradien von $1.2-1.3 \AA$, zu deren Erklärung Molecular-Modeling-Studien durchgeführt wurden.

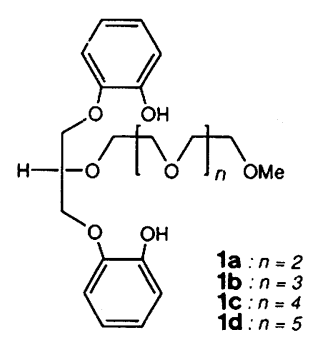

J. S. Bartlett, J. F. Costello, S. Mehani,

S. Ramdas, A. M. Z. Slawin,

J. F. Stoddart*, D. J. Williams

Angew. Chem. 102 (1990) 1463

1465

Neuartige Metalleinschlußverbindungen
Die Übertragung des $\mathrm{N}_{2} \mathrm{O}$-Sauerstoffatoms auf ein $\mathrm{Fe}\left(\mathrm{C}_{2} \mathrm{H}_{4}\right)^{\oplus}$-Ion unter Bildung des Metallacyclus 1 ist der Schlüsselschritt der Titelreaktion. Dies folgt aus Ionen-Cyclotron-Resonanzexperimenten ebenso wie aus mechanistischen und thermochemischen Überlegungen. Nach Zerfall des ,,heißen“ Intermediats $1 \mathrm{zu} \mathrm{Fe}{ }^{\oplus}$ und Acetaldehyd [Gl. (a)] kann ein neuer Katalysecyclus gestartet werden.

$1 \underset{\mathrm{H}_{2} \mathrm{C}-\stackrel{\mathrm{CH}}{2}_{2}^{\mathrm{Fe}}-\mathrm{O}}{\stackrel{\mathrm{O}}{\mathrm{C}}} \longrightarrow \mathrm{Fe}^{\oplus}+\mathrm{CH}_{3} \mathrm{CHO}$

(a)
D. Schröder, H. Schwarz*

Angew. Chem. 102 (1990) 1466. 1468

$\mathrm{Fe}^{\oplus}$-katalysierte Gasphasenoxidation von Ethan durch $\mathrm{N}_{2} \mathrm{O}$
- wobei im wesentlichen $\mathrm{FeOH}^{\oplus}+\mathrm{CH}_{3}^{-}$sowie $\mathrm{Fe}^{\oplus}+\mathrm{CH}_{3} \mathrm{OH}$ entstehen. Zwar läuft diese Reaktion [Gl. (a)] viermal langsamer als die in der vorstehenden Zuschrift beschriebene $\mathrm{ab}$, dennoch stehen die Chancen für eine katalytische Aktivierung nicht schlecht, da das im Zuge der reduktiven Eliminierung aus der Zwischenstufe 1 regenerierte $\mathrm{Fe}^{\oplus}$ durch $\mathrm{N}_{2} \mathrm{O}$ leicht wieder der Reaktion zugeführt werden kann.
D. Schröder, H. Schwarz*

Angew. Chem. 102 (1990) 1468. 1469

$\mathrm{FeO}^{\oplus}$ aktiviert Methan
$\mathrm{FeO}^{\oplus}+\mathrm{CH}_{4} \longrightarrow\left[\begin{array}{c}{\left[\mathrm{H}_{3} \mathrm{C}-\stackrel{\oplus}{\mathrm{Fe}}-\mathrm{OH}\right]} \\ 1\end{array} \longrightarrow \begin{array}{l}\longrightarrow \mathrm{Fe}^{\oplus}+\mathrm{CH}_{3} \mathrm{OH} \\ \mathrm{FeOH}^{\oplus}+\mathrm{CH}_{3}\end{array}\right.$ 
Lithiumsand statt Naphthalinkalium liefert bei der Synthese der Dianionen 1 und 2 aus den $\eta^{6}$-koordinierten Neutralkomplexen reine Produkte, da keine Möglichkeit zu Austauschreaktionen mit Naphthalin besteht. Auffällig an $\mathbf{2}$ ist, daß die gespannteste Doppelbindung der Cyclobutabenzol-Einheit auch nach der Reduktion an das Metallzentrum gebunden bleibt.

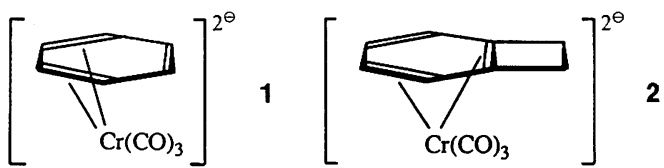

H. G. Wey, H. Butenschön*

Angew. Chem. 102 (1990) 1469

Darstellung und direkte NMR-spektroskopische Beobachtung von $\left[\left(\eta^{4}\right.\right.$-aren)$\mathrm{Cr}(\mathrm{CO})_{3}$ ]-Dianionen
Deutlich fester als Ge ${ }^{\text {II }}$ ist $\mathbf{S n}^{\text {II }}$ im Hohlraum des [2.2.2]Paracyclophans(L) gebunden, wie die Strukturanalysen der komplexen Kationen $\left[\mathrm{LSn}\left(\mathrm{AlCl}_{4}\right)\right]^{\oplus}$ und $[\mathrm{LGeCl}]^{\oplus} 1$ zeigen. Während im Zinnkomplex das $\mathrm{AlCl}_{4}^{\ominus}-\mathrm{Ge}$ genion nur locker über ein Cl-Atom am Metallzentrum koordiniert ist ( $\mathrm{Sn}-\mathrm{Cl} 3.073 \AA$ ) und damit die erwartete trigonal-planare Koordination nur unwesentlich stört, führt die starke $\mathrm{Ge}-\mathrm{Cl}$-Bindung $(\mathrm{Ge}-\mathrm{Cl} 2.224 \AA)$ in 1 zu einer Abschwächung der Ge-ArenWechselwirkung und einer eher tetraedrischen Umgebung des Metallzentrums.

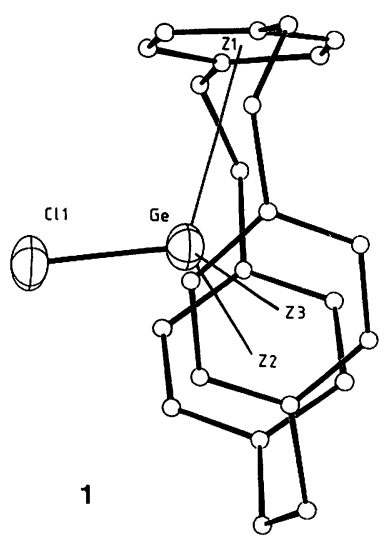

T. Probst, O. Steigelmann, J. Riede, H. Schmidbaur*

Angew. Chem. 102 (1990) 1471 1473

$\mathrm{Ge}^{\mathrm{Il}}$ - und $\mathrm{Sn}^{\mathrm{Il}}$-Komplexe des [2.2.2]Paracyclophans mit dreifacher interner $\eta^{6}-\mathrm{Ko}-$ ordination
Über Chelatliganden verbrückte Digoldkomplexe eignen sich ebenfalls zur ,molekularen Vergoldung" von Kohlenstoffatomen, wie die Struktur des $\mathrm{CAu}_{6}$-Komplexkations 1 zeigt. Aufgrund der Asymmetrie der Ligandensphäre sind die Kationen chiral. Die Bildung von 1 demonstriert, daß das Aurophilie-Konzept auch auf zweizähnige Liganden erweitert werden kann.

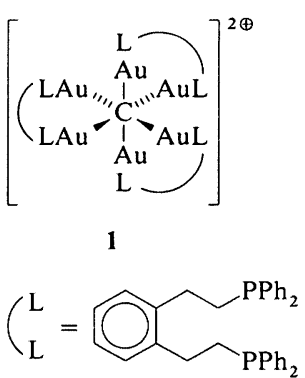

O. Steigelmann, P. Bissinger,

H. Schmidbaur*

Angew. Chem. 102 (1990) 1473 1475

Aufbau des $\left[\mathrm{CAu}_{6}\right]^{2 \oplus}$-Clusters mit einem maßgeschneiderten, die Oktaederkanten überspannenden Diphosphan
Die erste Verletzung der Hundschen Regel in einer Gleichgewichtsstruktur legen quantenchemische Rechnungen für $\mathrm{C}_{2} \mathrm{~S}_{2}$ nahe, denn sie liefern entgegen der Erwartung einen Singulett-Grundzustand. Die experimentellen Befunde der IR- und UV-spektroskopischen Untersuchung des in einer Ar-Matrix aus Vorläuferverbindungen wie $\mathbf{1}$ erhaltenen $\mathrm{C}_{2} \mathrm{~S}_{2}$ stimmen mit den Rechnungen gut überein.

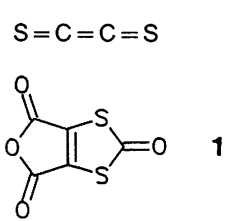

G. Maier*, H. P. Reisenauer, J. Schrot, R. Janoschek

Angew. Chem. 102 (1990) 1475

1477

$\mathrm{C}_{2} \mathrm{~S}_{2}$ (Ethen-1,2-dithion), ein Beispiel für die Verletzung der Hundschen Regel?
In $70 \%$ Ausbeute läßt sich das Dimer 1 durch Glaser-Kupplung herstellen, wenn die Reaktion in Gegenwart von 4,4'-Bipyridin als Templat durchgeführt wird. Mit einem Tripyridyltriazin als Templat entsteht bevorzugt das entsprechende Trimer (52\%). Ohne Templat verläuft die Reaktion unselektiv: Dimer und Trimer entstehen nebeneinander in 2025 bzw. 30-35\% Ausbeute. Die Ausbeuten korrespondieren gut mit den Bindungseigenschaften dieser Käfigmoleküle, die als Vorstufen für Enzymmodelle von Interesse sind.

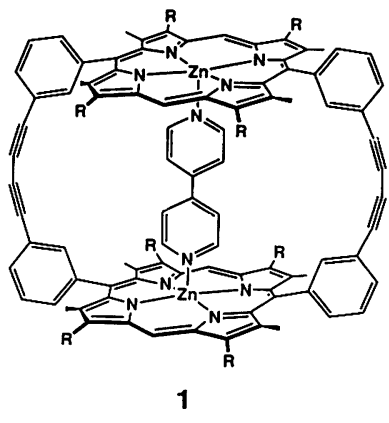

H. L. Anderson, J. K. M. Sanders*

Angew. Chem. 102 (1990) 1478 1480

Synthese cyclischer Porphyrin-Oligomere mit Aminen als Templaten 
Sehr unterschiedliche Strukturen haben die dinuclearen Natriumamid-Addukte 1 und 2: In 1 sind die zweizähnigen Amid-Ionen terminal an die $\mathrm{Na}^{\oplus}$-Ionen gebunden, in 2 verbrücken sie diese. Darüber hinaus ist bemerkenswert, daß HMPA in 1 verbrückend wirkt und daß in $\mathbf{2}$ das dreizähnige PMDETA nur mit zwei $\mathrm{N}$-Atomen an $\mathrm{Na}^{\oplus}$ koordiniert ist.

$\left[\mathrm{Ph}\left(2-\mathrm{NC}_{5} \mathrm{H}_{4}\right) \mathrm{N}\right] \mathrm{Na} \cdot 3 \mathrm{HMPA} \cdot \mathrm{Na}\left[\mathrm{N}\left(2-\mathrm{NC}_{5} \mathrm{H}_{4}\right) \mathrm{Ph}\right]: 1$

$\left[\left\{\mathrm{Ph}\left(2-\mathrm{NC}_{5} \mathrm{H}_{4}\right) \mathrm{N}\right\} \mathrm{Na} \cdot \mathrm{PMDETA}\right]_{2} \quad 2$
P. C. Andrews, W. Clegg, R. E. Mulvey*

Angew. Chem. 102 (1990) 1480...1481

Lewis-Basen-gesteuerte Strukturvarianten in der Natriumamid-Chemie: Röntgenstrukturanalysen von Phenyl(2-pyridyl)natriumamid-Addukten mit Hexamethylphosphorsäuretriamid und mit Pentamethyldiethylentriamin
Ringstapelstrukturen weisen die oligomeren Natriumamide 1-3 auf, die bei Versuchen zur Metallierung von Kohlenwasserstoffen durch $n$-Butylnatrium in Gegenwart von Tetramethylethylendiamin entstanden. Entsprechend gebaute Lithiumamide sind nicht bekannt; vielmehr sollten hier Leiterstrukturen bevorzugt sein.

$\left[\mathrm{Na}_{10}\left(\mathrm{NMe}_{2}\right)_{10}(\text { tmeda })_{4}\right]\left[\mathrm{Na}_{12}\left(\mathrm{NMe}_{2}\right)_{12}(\mathrm{tmeda})_{4}\right]\left[\mathrm{Na}_{12}\left(\mathrm{NMe}_{2}\right)_{10}\left(\mathrm{CH}_{2} \mathrm{C}_{6} \mathrm{H}_{4} \mathrm{Me}\right)_{2}(\mathrm{tmeda})_{4}\right]$
N. P. Lorenzen, J. Kopf, F. Olbrich, U. Schümann, E. Weiss*

Angew. Chem. 102 (1990) 1481 .1484

Decamere und dodecamere Natriumamide sowie ein Organonatriumamid mit Stapelstrukturen: $\left.\left[\mathrm{Na}_{10}\left(\mathrm{NMe}_{2}\right)_{10} \text { (tmeda }\right)_{4}\right]$, $\left.\left[\mathrm{Na}_{12}\left(\mathrm{NMe}_{2}\right)_{12} \text { (tmeda) }\right)_{4}\right]$ und $\left[\mathrm{Na}_{12}-\right.$ $\left(\mathrm{NMe}_{2}\right)_{10}\left(\mathrm{CH}_{2} \mathrm{C}_{6} \mathrm{H}_{4} \mathrm{Me}\right)_{2}$ (tmeda $\left.)_{4}\right]$
Die Stapelung von Arylgruppen scheint einmal mehr strukturbestimmend $\mathrm{zu}$ sein. Der Titelkomplex, der durch Umsetzung von $\left[\mathrm{Zn}\left(\mathrm{CH}_{2} \mathrm{SiMe}_{3}\right)_{2}\right]$ mit zwei Äquivalenten $2,4,6-t \mathrm{BuC}_{6} \mathrm{H}_{2} \mathrm{SH}$ in Gegenwart von $\mathrm{Et}_{2} \mathrm{O}$ zugänglich ist, hat einen S-Zn-S-Winkel von ca. $160^{\circ}$ und $\mathrm{Zn}$-S-Abstände von nur $2.196 \AA$. Der $\mathrm{Zn}-\mathrm{O}-\mathrm{Abstand}$ ist hingegen relativ groß und erklärt die leichte Abspaltbarkeit der Ether-Liganden. Zinkthiolate sind als Modellkomplexe für zinkhaltige Proteine von Interesse.

\section{P. P. Power*, S. C. Shoner}

Angew. Chem. 102 (1990) 1484...1485

Synthese und Struktur von $\left[\mathrm{Et}_{2} \mathrm{OZn}\right.$ $\left.\left(\mathrm{SC}_{6} \mathrm{H}_{2} t \mathrm{Bu}_{3}\right)_{2}\right]$, dem ersten $\mathrm{T}$-förmigen Zink-Komplex
Effiziente chirale Protonenquellen sind schon lange ein Wunschtraum der Organiker. Mit dem auf der Kempschen Tricarbonsäure basierenden Lactam 1 konnten jetzt cyclische Enolate mit einem Enantiomerenüberschuß (ee) von bis zu $91 \%$ protoniert werden. Ursache der hohen $e e$-Werte ist die asymmetrische Mikroumgebung der sauren $\mathrm{NH}$ Gruppe.

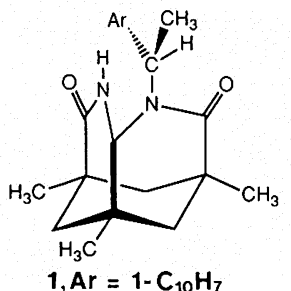

D. Potin, K. Williams, J. Rebek, Jr.*

Angew. Chem. 102 (1990) 1485 1486

Asymmetrische Protonierung von Enolaten

Mehrere Wochen in Lösung stabil sind die carbenähnlichen Verbindungen 1, die aus Phosphanyldiazomethanen bereits bei $T \leq 35^{\circ} \mathrm{C}$ nahezu quantitativ entstehen, wenn der Tetramethylpiperidin(tmp)-Rest einer der Substituenten am Phosphor ist. Die Verbindungen 1 reagieren mit Ausnahme von 1 a mit $t$ BuNC zu den Keteniminen 2. a, $\mathrm{R}=\mathrm{N} i \mathrm{Pr}_{2}, \mathrm{R}^{\prime}=\mathrm{Me} ; \mathbf{b}, \mathrm{R}=\mathrm{NMe}_{2}, \mathrm{R}^{\prime}=\mathrm{Me}$; , $\mathrm{R}=\mathrm{NMe}_{2}, \mathrm{R}^{\prime}=i \mathrm{Pr} ; \mathbf{d}, \mathrm{R}=\mathrm{Ph}, \mathrm{R}^{\prime}=\mathrm{Me}$.

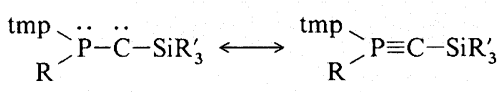

1<smiles>[R]P([As])C([As])=C=NCC</smiles>

2
G. R. Gillette, A. Baceiredo, G. Bertrand*

Angew. Chem. 102 (1990) 1486

Spontane Bildung stabiler Phosphino(silyl)carbene aus instabilen Diazoverbindungen

\footnotetext{
Mikrobiell nicht oder nur schwer abbaubare Chlorphenole und methoxylierte Arene werden in wenigen Minuten von einem Peroxidase-Modellsystem zu Chinonen oxidiert. Dieses Modellsystem wird durch Umsetzung sulfonierter Metalloporphyrin-Komplexe mit $\mathrm{KHSO}_{5}$ erhalten. Es oxidiert auch noch Arene wie 1,3,5-Trimethoxybenzol, das weder von Ligninase noch von Meerrettich-Peroxidase angegriffen wird.
}

G. Labat, J. L. Seris, B. Meunier*

Angew. Chem. 102 (1990) 1488... 1490

Oxidativer Abbau aromatischer Schadstoffe durch chemische Ligninase-Modelle auf Porphyrin-Basis 
Der Temperaturbereich der Mesophasen der Carboxylatokomplexe $\left[\mathrm{Mo}_{2}\left(\mathrm{O}_{2} \mathrm{CC}_{\mathrm{n}} \mathrm{H}_{2 \mathrm{n}+1}\right)_{4}\right]$ 1 hängt deutlich von der Länge der Kohlenwasserstoffkette $a b$. So geht $1, n=5$ (Hexanoat), bei $108^{\circ} \mathrm{C}$ in die Mesophase und erst bei $172^{\circ} \mathrm{C}$ in die isotrope Flüssigkeit über. Bei 1, $\mathrm{n}=9$ (Decanoat), betragen diese Werte 105 bzw. $116^{\circ} \mathrm{C}$ und bei $1, n=11$ (Dodecanoat), tritt keine Mesophase mehr auf.

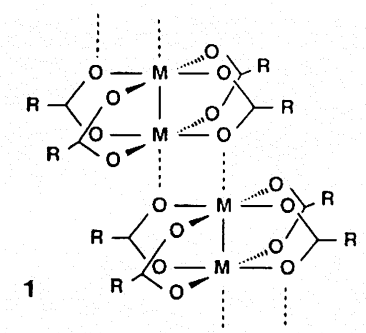

R. H. Cayton, M. H. Chisholm*, F. D. Darrington

Angew. Chem. 102 (1990) 1490

1491

Mehrfachbindungen zwischen Metallatomen in geordneten Aggregaten: Flüssigkristalle mit Mo-Mo-Vierfachbindungssystemen
Ein Dreieck aus Ba-Ionen mit zwei überdachenden $\mu_{3}$ - und drei kantenverbrückenden $\mu_{2}-\mathrm{OSiPh}_{3}$-Liganden liegt in der zentralen Struktureinheit (Bild rechts) der Titelverbindung vor. Alle drei Ba-Ionen sind jedoch unterschiedlich koordiniert: So sorgt an $\mathrm{Ba} 3$ ein terminaler Siloxido-Ligand für Ladungsausgleich, während ein THF-Ligand an Ba1 gebunden ist. Der Dreikernkomplex bildet sich in $77 \%$ Ausbeute beim Einleiten von $\mathrm{NH}_{3}$ in eine Reaktionsmischung aus Ba-Granalien und $\mathrm{Ph}_{3} \mathrm{SiOH}$ in THF.

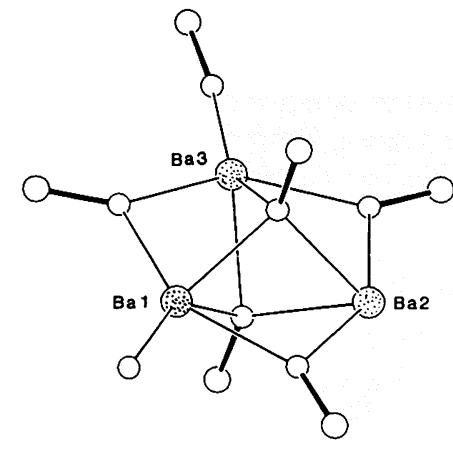

K. G. Caulton*, M. H. Chisholm, S. R. Drake, W. E. Streib

Angew. Chem. 102 (1990) 1492 1493

$\left[\mathrm{Ba}_{3}\left(\mathrm{OSiPh}_{3}\right)_{6}(\mathrm{THF})\right] \cdot 0.5 \mathrm{THF}-\mathrm{Syn}-$ these und Struktur eines dreikernigen $\mathrm{Ba}$ riumsiloxids mit niedrig koordiniertem Barium
Trotz starker Verdrillung des konjugierten Systems reagiert das Tetrathiafulvalen-Homologe 1 mit dem Elektronenacceptor Tetracyanchinodimethan zum Dikation $1^{2 \oplus}$. In $\mathbf{1}^{2 \oplus}\left[(\mathrm{TCNQ})_{4}\right]^{2 \ominus}$ ist das Anthracengerüst eingeebnet, und die Dithiolringe sind $86^{\circ}$ gegen die Anthracenebene verdrillt. Die 1:4Stöchiometrie des hoch leitfähigen Salzes $\left(\sigma_{300 \mathrm{~K}}=60 \mathrm{~S} \mathrm{~cm}^{-1}\right)$ ist für TCNQ-Komplexe sehr ungewöhnlich.

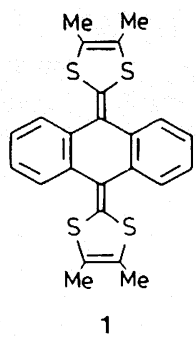

M. R. Bryce*, A. J. Moore, M. Hasan, G. J. Ashwell*, A. T. Fraser, W. Clegg, M. B. Hursthouse, A. I. Karaulov

Angew. Chem. 102 (1990) 1493 1495

Röntgenstrukturanalyse sowie elektrische und magnetische Eigenschaften eines hoch leitfähigen 4:1-Komplexes aus Tetracyanchinodimethan und einem Tetrathiafulvalen-Homologen
Eine empfindliche Sonde für die Bildung der anionischen $\mathrm{N}_{\mathbf{2}}$-Komplexe 1 und 2 bei der Reaktion von $\left[\mathrm{VCl}_{3}(\text { thf })_{3}\right]$ mit $\mathrm{N}_{2}$, dmpe und $\mathrm{Na}$ ist ihr Zentralmetall. So ist das ${ }^{51} \mathrm{~V}$ NMR-Signal des trans-Komplexes 1 ein Quintett bei $\delta=-1123$. Wird die Reduktion unter ${ }^{15} \mathrm{~N}_{2}$ durchgeführt, so spalten die einzelnen Multiplett-Komponenten zusätzlich zu Tripletts auf. Damit liegt ein direkter Nachweis für den Einbau von Stickstoff in den Komplex vor. dmpe = Dimethylphosphinoethan.

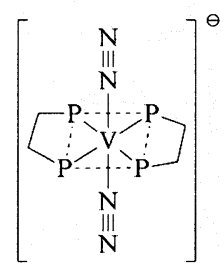

1

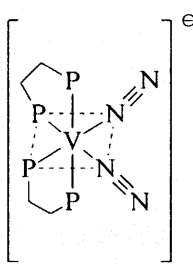

2
C. Woitha, D. Rehder*

Angew. Chem. 102 (1990) 1495.

.1497

Vanadium(-I)-Stickstoffkomplexe mit endon koordiniertem $\mathrm{N}_{2}$ : funktionelle Modelle für die ,,alternative Nitrogenase“
Deutliche Hinweise auf den Einschluß von Pyranosiden in den Makrocyclus $1(\mathrm{R}=\mathrm{H}$, $\mathrm{R}^{\prime}=\mathrm{Bn}$ ) lieferten NMR- und IR-spektroskopische Untersuchungen der Umsetzung mit 2 in $\mathrm{CDCl}_{3}$ sowie Kraftfeldrechnungen für das Methylanalogon von 2. Bei 1 handelt es sich somit um einen der ersten künstlichen Kohlenhydrat-Rezeptoren. Sein Hohlraum ist groß genug, um das Gastmolekül von allen Seiten durch gerichtete H-Brücken zu fixieren.

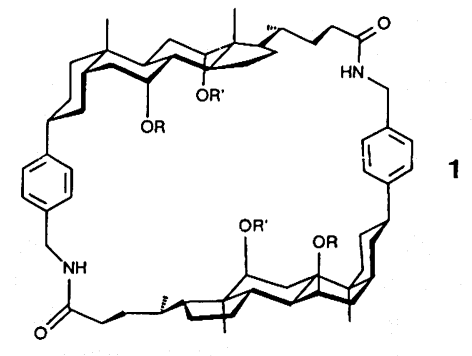

R. P. Bonar-Law, A. P. Davis*, B. A. Murray

Angew. Chem. 102 (1990) 1497 1499

Künstliche Rezeptoren für Kohlenhydratderivate 
Durchschnittlich 110 verknüpfte Metallomakrocyclen liegen in einem Oligomerstapel vor, wenn der Phthalocyaninatoeisen-Komplex 1, $\mathrm{R}^{1}=\mathrm{R}^{2}=2-\mathrm{Et}-\mathrm{C}_{6} \mathrm{H}_{12}$, mit Benzoldiisocyanid umgesetzt wird. Dieses und andere Oligomere konnten durch ${ }^{57} \mathrm{Fe}-\mathrm{Mößbauer}$ - und NMR-Spektroskopie charakterisiert werden.

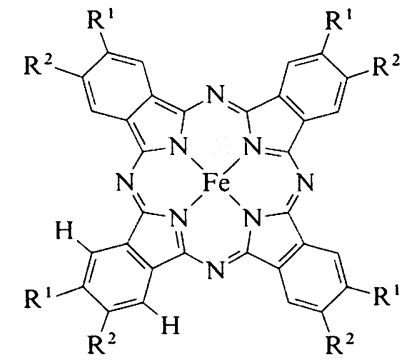

M. Hanack*, A. Hirsch, H. Lehmann

Angew. Chem. 102 (1990) 1499.

.1501

Lösliche, oligomere verbrückte Phthalocyaninatoeisen(II)-Komplexe

\begin{abstract}
${ }^{31} \mathrm{P}$-NMR-chemische Verschiebungen von $\delta=$ 3471 (!) und $\delta=727$ spiegeln die ungewöhn-

D. Baudry*, M. Ephritikhine, F. Nief*, L. Ricard, F. Mathey
\end{abstract} liche Bindungssituation im dimeren, paramagnetischen Organouran(III)-Komplex 1 wider. Das extrem tieffeldverschobene Signal kann dem P-Atom des verbrückenden Phospholylliganden zugeordnet werden. 1 läßt sich mit $\mathrm{TlBH}_{4}$ zum Uran(IV)-Sandwich $\left[\left(\mathrm{C}_{4} \mathrm{Me}_{4} \mathrm{P}\right)_{2} \mathrm{U}\left(\mathrm{BH}_{4}\right)_{2}\right]$ oxidieren, der bei Reduktion mit Na-Amalgam wieder 1 ergibt.

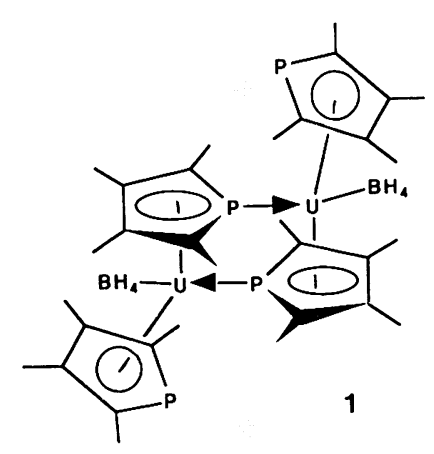

Angew. Chem. 102 (1990) 1501.

..1502

Synthese von Phospholyl(tetrahydroborato)uran-Komplexen. Kristallstruktur von $\left[\left(\eta^{5}-\mathrm{C}_{4} \mathrm{Me}_{4} \mathrm{P}\right)_{2} \mathrm{U}\left(\mathrm{BH}_{4}\right)_{2}\right]$
Ein Nitrit-analoges, niederkoordiniertes Phosphorchalcogenid ist das überraschendste Strukturmerkmal des anionischen Wolframkomplexes 1. Die gewinkelte $\mathrm{PSe}_{2}^{\ominus}$-Einheit ist seitlich an das Wolfram gebunden, ein terminaler Seleno- und ein zweizähniger, tetraedrischer $\mathrm{PSe}_{4}^{3 \Theta}$-Ligand vervollständigen die Koordinationssphäre des Metallzentrums.

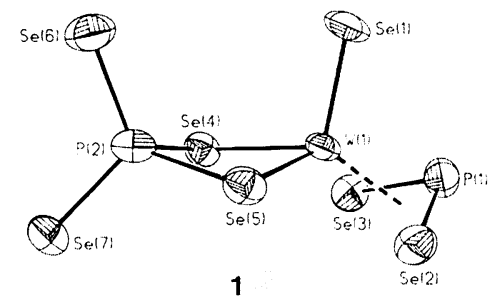

S. C. O'Neal, W. T. Pennington, J. W. Kolis*

Angew. Chem. 102 (1990) 1502. 1504

Synthese und Struktur von $\left[\mathrm{Se}=\mathrm{W}\left(\mathrm{PSe}_{4}\right)\left(\mathrm{PSe}_{2}\right)\right]^{2 \ominus}$; ein Dianion mit einer heteroallylischen $\mathrm{PSe}_{2}^{\ominus}$-Einheit
Nicht nur von ästhetischem Reiz ist der $\mathrm{Al}_{\mathbf{4}} \mathrm{P}_{\mathbf{4}}$ Würfel in $\left[i \mathrm{BuAl}\left(\mu_{3}-\mathrm{PSiPh}_{3}\right)\right]_{4}$ 1. Sein Verhalten bei thermischer Belastung macht ihn auch als Vorläufer für den III/V-Halbleiter AIP interessant. Bereits bei $150^{\circ} \mathrm{C}$ werden die Substituenten als $\mathrm{Ph}_{3} \mathrm{SiH}$ und Isobuten abgespalten, Erhöhung der Temperatur auf $500^{\circ} \mathrm{C}$ führt zu reinem AlP.

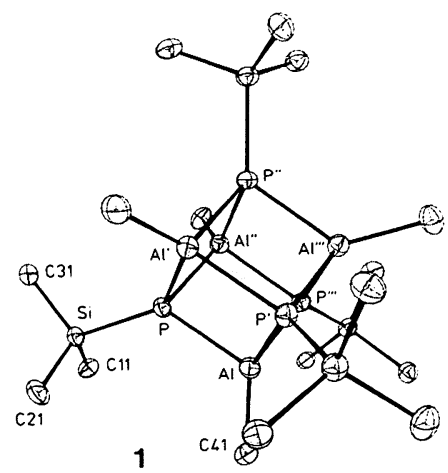

A. H. Cowley*, R. A. Jones*, M. A. Mardones, J. L. Atwood*, S. G. Bott

Angew. Chem. 102 (1990) 1504. 1505

Ein Aluminaphosphacuban, ein neuer Vorläufer für Aluminiumphosphid
Bei der Koordination an Metallzentren nehmen 1,3-Diene sehr häufig eine s-cis-Konformation an. Der Ru-Komplex 1 ist der erste kationische s-trans-Dien-Komplex, der bei Raumtemperatur in Lösung stabil ist. Derartige Komplexe sind als reaktive Intermediate bei der katalytischen Umsetzung konjugierter Diene denkbar.

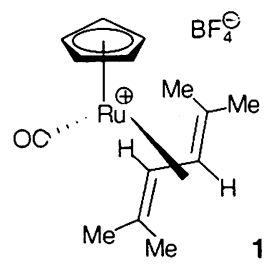

S. A. Benyunes, J. P. Day, M. Green*, A. W. Al-Saadoon, T. L. Waring

Angew. Chem. 102 (1990) 1505.

1507

s-trans-1,3-Diene als Liganden für MetallKationen der 8. Gruppe $\pi / \sigma$-Verhältnisse von $>96:<4$ bis $15: 85$ (bei 299 K) treten in den Komplexen 1 in Abhängigkeit vom Arylsubstituenten auf. Neben diesem starken elektronischen Einfluß konnten auch deutliche Lösungsmittel- und Temperatureinflüsse nachgewiesen werden. Diese Befunde sind für das Verständnis von Addukten aus Carbonylverbindungen und Metallkomplex-Fragmenten von großer Bedeutung.

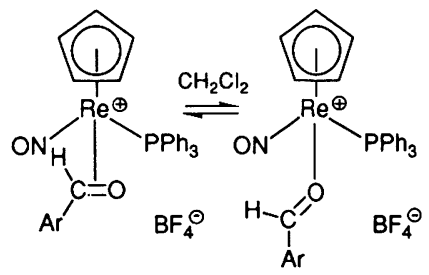

1
N. Q. Méndez, A. M. Arif, J. A. Gladysz*

Angew. Chim. 102 (1990) 1507. .1509

$\pi / \sigma$-Gleichgewichte in Metall-Komplexen organischer Carbonylverbindungen: Synthese und Struktur chiraler RheniumKomplexe $\quad\left[\left(\eta^{5}-\mathrm{C}_{5} \mathrm{H}_{5}\right) \mathrm{Re}(\mathrm{NO})\left(\mathrm{PPh}_{3}\right)\right.$ $(\mathrm{O}=\mathrm{CHAr})] \mathrm{X}$ 
Je höher die $\pi$-Acidität des Aldehyd-Liganden desto ausgeprägter die chirale Erkennung bei seiner Bindung an das chirale Komplexfragment 1. Dies ist das Ergebnis Tieftemperatur-NMR-spektroskopischer Untersuchungen der Komplexe von 1 mit den aromatischen Aldehyden 2, die zudem zeigten, daß sich die diastereomeren $\pi$-Aldehyd-Komplexe aus $\mathbf{1}$ und $\mathbf{2}$ intramolekular über einen $\sigma$-Aldehyd-Komplex ineinander umwandeln.

$\left[\left(\eta^{5}-\mathrm{C}_{5} \mathrm{H}_{5}\right) \operatorname{Re}(\mathrm{NO})\left(\mathrm{PPh}_{3}\right)\right]^{\oplus} 1$

Ar-CHO 2, $\mathrm{Ar}=\mathrm{C}_{6} \mathrm{~F}_{5}, 4-\mathrm{CF}_{3} \mathrm{C}_{6} \mathrm{H}_{4}$,

4- $\mathrm{ClC}_{6} \mathrm{H}_{4}, \mathrm{C}_{6} \mathrm{H}_{5}$,

4- $\mathrm{CH}_{3} \mathrm{C}_{6} \mathrm{H}_{4}, 4-\mathrm{H}_{3} \mathrm{COC}_{6} \mathrm{H}_{4}$

N. Q. Méndez, C. L. Mayne,

J. A. Gladysz*

Angew. Chem. 102 (1990) 1509

1511

Thermodynamisch kontrollierte enantiofaciale Selektivität bei der Bindung von Aldehyden an chirale MetallkomplexFragmente - der Mechanismus der Interkonversion diastereomerer $\pi$-AldehydKomplexe $\quad\left[\left(\eta^{5}-\mathrm{C}_{5} \mathrm{H}_{5}\right) \mathrm{Re}(\mathrm{NO})\left(\mathrm{PPh}_{3}\right)\right.$ $\left(\eta^{2}-\mathrm{O}=\mathrm{CHAr}\right) \mathrm{BF}_{4}$

\section{J. Burk*, R. L. Harlow}

Angew. Chem. 102 (1990) 1511

1513

Neue chirale Tripod-Phosphane mit $\mathrm{C}_{3}$ Symmetrie zugänglich sind, wurden als Liganden in Rh-Komplexen untersucht.
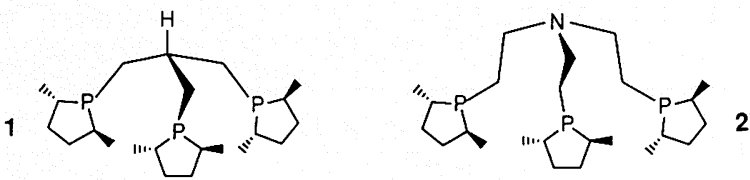

Der bisher größte röntgenkristallographisch charakterisierte Cluster, die Verbindung 1, entsteht zusammen mit wenig 2 (Struktur: Bild rechts) bei der Umsetzung von $\mathrm{CuCl}$ mit $\mathrm{Se}\left(\mathrm{SiMe}_{3}\right)_{2}$ und $\mathrm{PEt}_{3}$. Zwischenstufe auf dem Weg zu 1 könnte ein $\mathrm{Cu}_{9}$-Cluster sein. Beim Erhitzen von 1 auf über $160^{\circ} \mathrm{C}$ bildet sich $\mathrm{Cu}_{2} \mathrm{Se}$.

$\left[\mathrm{Cu}_{70} \mathrm{Se}_{35}\left(\mathrm{PEt}_{3}\right)_{22}\right] \quad 1$

$\left[\mathrm{Cu}_{20} \mathrm{Se}_{13}\left(\mathrm{PEt}_{3}\right)_{12}\right] \quad 2$

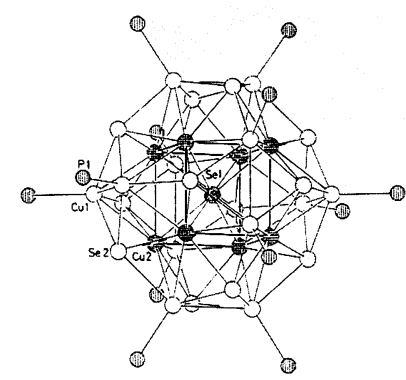

D. Fenske*, H. Krautscheid

Angew. Chem. 102 (1990) 1513

1516

Neue Kupfercluster mit Se und $\mathrm{PEt}_{3}$ als Liganden: $\quad\left[\mathrm{Cu}_{70} \mathrm{Se}_{35}\left(\mathrm{PEt}_{3}\right)_{22}\right]$ und $\left[\mathrm{Cu}_{20} \mathrm{Se}_{13}\left(\mathrm{PEt}_{3}\right)_{12}\right]$
Nicht aromatisch, sondern chinoid ist das energetisch günstigste Isomer des in der Gasphase nachgewiesenen $\mathrm{C}_{6} \mathrm{~S}_{6}$. Die Energiedifferenz zwischen $\mathbf{A}$ und $\mathbf{B}$ wurde durch ab-initio-Rechnungen zu $10.9 \mathrm{kcal} \mathrm{mol}^{-1}$ bestimmt. Nahezu energiegleich mit $\mathbf{B}$ ist dagegen das Hexathioketon $\mathbf{C}$, das in einer Sesselkonformation vorliegen sollte.<smiles></smiles>

A<smiles></smiles><smiles>S=C1C(=S)C(=S)C(=S)C(=S)C1=S</smiles>

C
G. Frenking*

Angew. Chem. 102 (1990) 1516 . .1517

Die Struktur von cyclischem $\mathrm{C}_{6} \mathrm{~S}_{6}$ und $\mathrm{C}_{6} \mathrm{O}_{6}$
Ein gewellter sechsgliedriger $\mathbf{P t}_{\mathbf{3}} \mathbf{C l}_{\mathbf{3}}-\mathrm{Ring}$, der über seine basischen Pt-Zentren ein ,nacktes" Sn ${ }^{\text {II }-Z e n t r u m ~ k o o r d i n i e r t, ~ s o ~ l a ̈ ß t ~ s i c h ~}$ der Clusterkern (Bild rechts) der Titelverbindung beschreiben. Außer den Pt-Sn-Wechselwirkungen tragen noch kurze Kontakte zwischen $\mathrm{Sn}^{\mathrm{II}}$ und den $o$-F-Atomen $\operatorname{der} \mathrm{C}_{6} \mathrm{~F}_{5}$ Liganden am Platin zur Stabilisierung dieses ungewöhnlichen Komplexanions bei

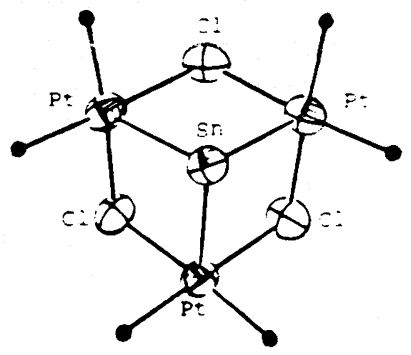

R. Usón*, J. Forniés, M. Tomás, I. Usón

$\left(\mathrm{NBu}_{4}\right)\left[\mathrm{Sn}\left\{\mathrm{Pt}(\mu-\mathrm{Cl})\left(\mathrm{C}_{6} \mathrm{~F}_{5}\right)_{2}\right\}_{3}\right]$, ein ungewöhnlicher Cluster mit drei $\mathrm{Pt}^{1 \mathrm{II}} \rightarrow \mathrm{Sn}^{\mathrm{II}}$ Bindungen 
Bei $80^{\circ} \mathrm{C}$ nimmt Caesiumfluorid Brom auf, und zwar im Verhältnis 1:1. Dabei weitet sich das Wirtsgitter parallel zu einer der (100)Flächen auf (Bild rechts). Der Verlust an Gitterenergie wird bereits durch eine sehr schwache $\mathrm{Br} \cdots \mathrm{F}^{\ominus}$-Wechselwirkung in der Größe von wenigen $\mathrm{kJ} \mathrm{mol}^{-1}$ kompensiert.

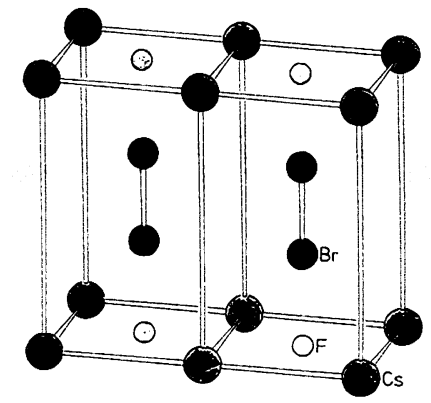

D. D. DesMarteau, T. Grelbig,

S. H. Hwang, K. Seppelt*

Angew. Chem. 102 (1990) 1519.

$\mathrm{CsF} \cdot \mathrm{Br}_{2}$, eine Alkalimetallhalogenid-Intercalationsverbindung
Stabil selbst gegen starke Säuren ist die Palocgeschützte Aminofunktion in Dipeptidestern wie 1. Dies ermöglicht die selektive, quantitative Abspaltung der $t \mathrm{Bu}-\mathrm{Gruppe}$ mit $\mathrm{HCl}$ in $\mathrm{Et}_{2} \mathrm{O} / \mathrm{CH}_{2} \mathrm{Cl}_{2}$. Umgekehrt gelingt die gezielte Ablösung der Paloc-Schutzgruppe durch Palladium(0)-katalysierte Allylübertragung auf schwach basische Nucleophile wie $N$ Methylanilin. Bei dieser Reaktion bleiben viele Schutzgruppen intakt, d.h. empfindliche Strukturen werden nicht zerstört.
Paloc-Ile-Phe-O $t$ Bu 1<smiles>CC(=O)OC/C=C/c1cccnc1</smiles>

K. von dem Bruch, H. Kunz*

Angew. Chem. 102 (1990) 1520.

1522

Der 3-(3-Pyridyl)allyloxycarbonyl(Paloc)Rest - eine stabile, unter neutralen Bedingungen abspaltbare Aminoschutzgruppe für Peptidsynthesen in organischen Medien und in Wasser
Wittig-Reaktionen mit semistabilisierten Yliden verlaufen meist stereounselektiv. Unter konsequenter Berücksichtigung bekannter Fakten wurden nun die Benzyl-Ylide 1 a und 1 b hergestellt und mit Aldehyden umgesetzt. Das Arsen-Ylid $\mathbf{1 b}$ lieferte bei der Reaktion mit Piperonal und Hexanal jeweils $100 \%$ des (E)-Olefins (GC-Analyse).

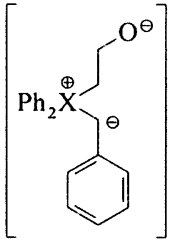

1 a: $X=P$

b: $X=A s$
B. Boubia, A. Mann, F. D. Bellamy, C. Mioskowski*

Angew. Chem. $102(1990) 1522$

1523

Neue Benzyl( $\beta$-oxido)phosphor- und -arsen-Ylide: Reaktivität und Stereoselektivität bei der Wittig-Reaktion
Photochemisch lagert sich das Silyl-substituierte $\mathbf{1 H}$-Phosphiren 1 zum kristallinen Dihydrophosphasilet 2 um. Wird dieses am Phosphoratom acyliert, so entsteht durch spontane 1,3-Silylverschiebung 3, ein Heterocyclus mit einer 2-Phospha-1-3-dien-Einheit. $\mathrm{R}^{1}=\mathrm{R}^{3}=t \mathrm{Bu}, \mathrm{R}^{2}=\mathrm{Ph}$.

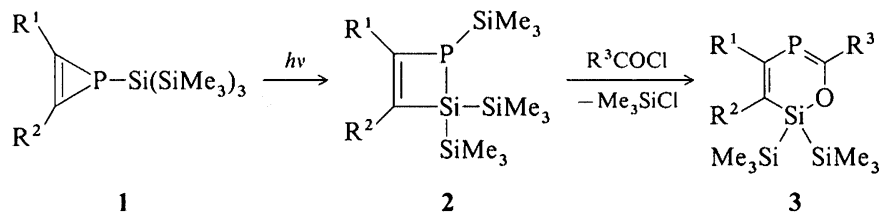

S. Haber, R. Boese, M. Regitz*

Angew. Chem. 102 (1990) 1523 .1525

Eine 1-Silyl-1 $H$-phosphiren/1,2-Dihydro1,2-phosphasilet-Umlagerung - der entscheidende Schritt zur Synthese von Heterocyclen mit 2-Phospha-1,3-dien-Einheiten

\begin{abstract}
Wegen ihrer Ähnlichkeit zu helicalen Polymeren und für einen Vergleich mit chiralen stabförmigen Flüssigkristallen sind chirale discotisch-flüssigkristalline Polymere interessant. Sie wurden nun auf mehrere Arten zugänglich gemacht, z. B. durch Dotierung achiraler Donor-Polymere mit chiralen Acceptoren (siehe Bild). Die Chiralität der discotischen Phasen wurde an dünnen Filmen CD-spektroskopisch untersucht.
\end{abstract}

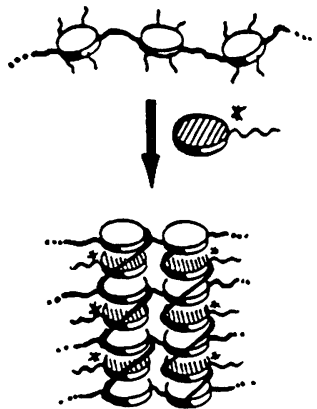

M. M. Green, H. Ringsdorf*, J. Wagner, R. Wüstefeld

Angew. Chem. 102 (1990) 1525

Induktion und Variation von Chiralität in discotisch-flüssigkristallinen Polymeren 
Die sequentielle Öffnung zweier vicinaler Epoxidgruppen durch Cuprat-Reagentien in einem Schritt ist die Schlüsselreaktion beim Aufbau der Stereotetraden 1 und 2. Als Edukt für beide Verbindungen, die in mehreren Ionophor-Antibiotica enthalten sind, dient D-Mannit.<smiles>[M]C(CO)C(O)C([M])CO</smiles>

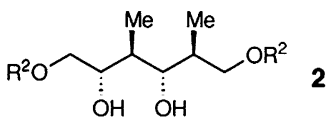

Brückenkopfolefin- und Brückenkopfalkohol-Einheit in einem Molekül entstehen gleichzeitig bei der Solvolyse des Mesylats 1 zu $2(R=H)$. Die Synthese von 1 aus Caryophyllenepoxid gelang erstmals stereoselektiv und in guter Ausbeute. Eine Röntgenstrukturanalyse des 4-Brombenzoats von 2 bestätigt die Strukturzuordnung.
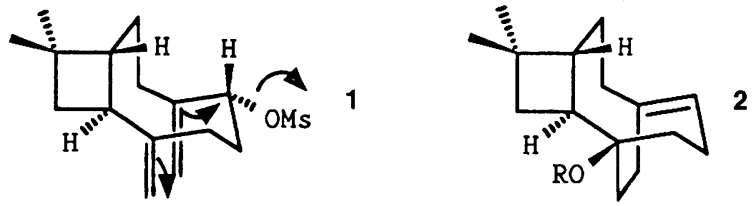

Eine Aktivierungsbarriere von $45 \mathrm{~kJ} \mathrm{~mol}^{-1}$ folgt aus den ${ }^{1} \mathrm{H}-\mathrm{NMR}$-Spektren für die erste nachgewiesene gehinderte Rotation um die $\mathrm{sp}^{2}-\mathrm{sp}^{2}$-Einfachbindung eines acyclischen $\alpha, \beta$-ungesättigten Ketons (1). Die Intensitäten der UV-Banden der $\pi \rightarrow \pi^{*}$ - und $n \rightarrow \pi^{*}$ Übergänge sind mit einer verdrillten Konformation von 1 in Einklang. Molekülmechanikrechnungen lassen allerdings eine deutlich höhere Aktivierungsbarriere erwarten, was die schlechte Parametrisierung für $\mathrm{sp}^{2}-\mathrm{sp}^{2}-$ Einfachbindungen belegt.
J. Mulzer*, B. Schöllhorn

Angew. Chem. 102 (1990) 1529 1530

Enantio- und regiokontrollierte Synthese eines zentralen Ionophor-AntibioticaBausteines durch sequentielle Öffnung zweier Epoxidringe mit Cuprat-Reagentien

U. Vogt, U. Eggert, A. M. Z. Slawin, D. J. Williams, H. M. R. Hoffmann*

Angew. Chem. 102 (1990) 1530

1532

Eine stereoselektive $\pi$-Cyclisierung zu einem kombinierten Brückenkopfolefin/ -alkohol

* Korrespondenzautor

\section{Neue Bücher}

\section{Chemiker als Literaten}

Cantor's Dilemma

C. Djerassi

Gaps and Verges

R. Hoffmann

pH-Messungen. Grundlagen, Methoden, Anwendungen, Geräte H. Galster

Aquatische Chemie

L. Sigg, W. Stumm

\section{Structure and Reactivity in Reverse Micelles}

M. P. Pileni

\section{Chemically Modified Carbon Fibers and their Applications}

I. N. Ermelenko, I. P. Lyubliner, N. V. Gulko

\section{Lectins}

N. Sharon, H. Lis

Mycotoxins. Chemical, Biological, and Environmental Aspects V. Betina

ABC Geschichte der Chemie

S. Engels, R. Stolz

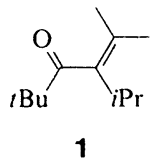

J. M. Nuss*, S. J. Bark, D. B. Borchardt, T. H. Morton*

Angew. Chem. 102 (1990) 1532 1533

Die sterisch gehinderte Rotation um die $C$-C-Bindung eines acyclischen $\alpha, \beta$-ungesättigten Ketons: 2,2,5-Trimethyl-4-isopropylhex-4-en-3-on
J. Heinze

Angew. Chem. 102 (1990) 1535

F. H. Frimmel

Angew. Chem. 102 (1990) 1536

A. Laschewsky

Angew. Chem. 102 (1990) 1536

W. Meyer

Angew. Chem. 102 (1990) 1537

H.-J. Gabius

Angew. Chem. 102 (1990) 1538

T. Anke

Angew. Chem. 102 (1990) 1538

G. Herrmann

Angew. Chem. 102 (1990) 1539 
T. Grandke, W. H. Ko

Vol. 5: Magnetic Sensors

R. Boll, K. J. Overshott

\section{Analytical NMR}

L. D. Field, S. Sternhell

Biomineralization, Chemical and Biochemical Perspectives

S. Mann, J. Webb, R. J. P. Williams

Molecular Structure of Organosilicon Compounds

E. Lukevics, O. Pudova, R. Sturkovich

Kunstoffverbundsysteme. Grundlagen, Anwendung, Verarbeitung, Prüfung

R. Janda

Schreiben und Publizieren in den Naturwissenschaften

H. F. Ebel, C. Bliefert

Houben-Weyl. Methoden der Organischen Chemie, C-Radikale, Teil 1 und 2

(Bände E 19 a)

M. Regitz, B. Giese
H. Kogler

Angew. Chem. 102 (1990) 1540

G. Krampitz

Angew. Chem. 102 (1990) 1541

M. Weidenbruch

Angew. Chem. 102 (1990) 1541

F. Kleiner

Angew. Chem. 102 (1990) 1542

L.-F. Tietze

Angew. Chem. 102 (1990) 1542

W.-D. Fessner

Angew. Chem. 102 (1990) 1543

Englische Fassungen aller Aufsätze und Zuschriften dieses Heftes erscheinen in der Dezember-Ausgabe der Angewandten Chemie International Edition in English. Entsprechende Seitenzahlen können einer Konkordanz im Januar-Heft der Angewandten Chemie entnommen werden.

\section{Chemiker-Biographien}

- ANGEWANDTE CHEMIE

- Chemische Berichte

- Liebigs Annalen der Chemie

In diesen drei traditionsreichen Zeitschriften steckt auch eine ganze Menge chemiehistorisches Material.

Als kostenlosen Service bietet die „Berichte/Annalen“"-Redaktion ein umfangreiches Verzeichnis an, das Zugang zu einigen hundert Nachrufen und ausführlichen biographischen Mitteilungen verschafft.

Das Verzeichnis finden Sie in Liebigs Annalen der Chemie (Heft 12,1990). Sonderdrucke sind beim Verlag erhältlich. Schreiben Sie an:

Redaktion Liebigs Annalen der Chemie

Postfach 101161 , W-6940 Weinheim

In den nächsten Heften werden unter anderem folgende Aufsätze erscheinen:

Moose, eine Quelle biologisch aktiver Naturstoffe?

H. D. Zinsmeister et al.

Auf dem Weg zur chemischen Synthese von Proteinen an polymeren Trägern - ein Beispiel für das Zusammenspiel der methodischen Entwicklung bei Synthese und Analyse

E. Bayer

Bioanorganische Chemie des Vanadiums

D. Rehder

Ribonuclease T1: Strukture, Funktion und Stabilität

W. Saenger et al.

Gepulste Elektronenspinresonanz-Spektroskopie: Grundlagen, Methodik und Anwendungsmöglichkeiten A. Schweiger 


\title{
Knüpfung von CC-Bindungen durch Addition von Carbenium-Ionen an Alkene: Kinetik und Mechanismus
}

\author{
Von Herbert Mayr * \\ Professor Rolf Huisgen zum 70. Geburtstag gewidmet
}

Die Addition von Carbenium-Ionen an CC-Doppelbindungen, ein Schlüsselschritt zahlreicher Synthesen in der Organischen und Makromolekularen Chemie, wird am Beispiel der LewisSäure-induzierten Umsetzungen von Alkylchloriden mit Alkenen mechanistisch analysiert. Stereochemische und kinetische Untersuchungen deuten einen wenig verbrückten, Produktähnlichen Übergangszustand an. Umlagerungen der im Additionsschritt erzeugten Carbenium-Ionen lassen sich durch Zusätze von Salzen mit nucleophilen Gegenionen zurückdrängen. Die Thermodynamik der Additionsreaktionen wird analysiert, und es wird gezeigt, unter welchen Voraussetzungen mit deren Umkehr, d. h. mit Grob-Fragmentierungen zu rechnen ist. Kinetische Untersuchungen über Struktureinflüsse auf die Reaktivität von Carbenium-Ionen und von Alkenen führen zu Mehrparameter-Gleichungen, die die Voraussage von Geschwindigkeitskonstanten ermöglichen. Reaktivitäts-Selektivitäts-Beziehungen über einen Reaktivitätsbereich von acht Zehnerpotenzen zeigen, daß die Struktur des Übergangszustands nur durch Substituenten-Variation in unmittelbarer Nähe des Reaktionszentrums verändert wird.

\section{Einleitung}

Lewis-Säure-induzierte CC-Verknüpfungen haben in den letzten Jahren große Bedeutung in der organischen Synthese erlangt $^{[1]}$. Im allgemeinen besteht die Aufgabe der LewisSäure darin, durch Komplexbildung mit einer Carbonylgruppe oder einem Imin oder durch Ionisation eines Acetals oder verwandter Verbindungen ein Elektrophil so stark zu aktivieren, daß es an einem elektronenreichen Doppelbindungssystem (z. B. Silylenolether, Allylmetallverbindungen) anzugreifen vermag (Schema 1).

Der Prototyp dieser Reaktionen ist die Addition eines Carbenium-Ions an eine gewöhnliche CC-Doppelbindung, wie sie bei der Lewis-Säure-induzierten Umsetzung von Alkylhalogeniden mit Alkenen und Dienen stattfindet (Schema 2). Da nunmehr die Voraussetzungen bekannt sind, unter

[*] Prof. H. Mayr

Institut für Chemie

Medizinische Universität zu Lübeck

Ratzeburger Allee 160, W-2400 Lübeck 1<smiles>[R6]C(C)=C(C)CC([Y])(C)C</smiles>

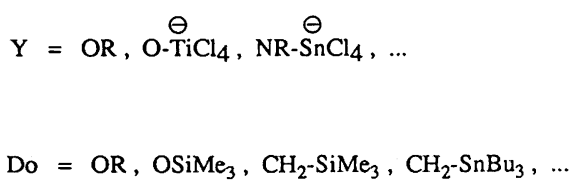

Schema 1.

denen solche Reaktionen gezielt, d.h. unter Vermeidung von Polymerisationen ablaufen ${ }^{[2]}$, sind mechanistische Untersuchungen dieser Reaktionsfolge möglich geworden.

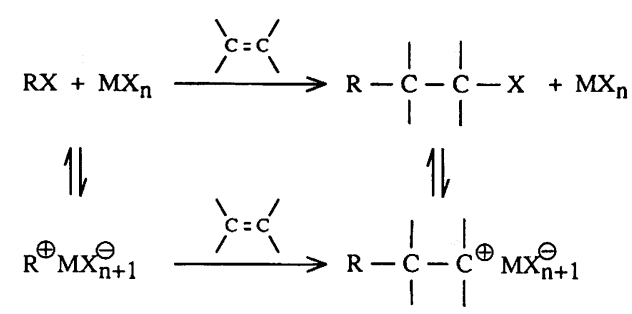

Schema 2. 
Als besonders geeignete Modellverbindungen erwiesen sich hierbei die $p$-substituierten Diarylcarbenium-Ionen 1, da sich deren Reaktivität durch Variation der Substituenten $X$ und $Y$ über weite Bereiche verschieben läßt, ohne daß die sterischen Verhältnisse am Reaktionszentrum verändert werden.
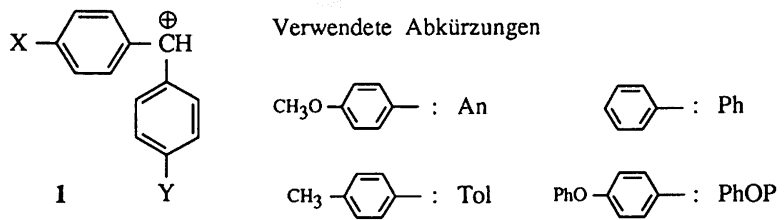

Die Carbenium-Ionen 1 lassen sich bei solchen Studien entweder in Form stabiler Salze einsetzen oder in Gegenwart der Alkene als kurzlebige Zwischenstufen in situ erzeugen.

\section{Regio- und Stereoselektivität}

Wie $\mathrm{H}^{\oplus}$-initiierte HX-Additionen ${ }^{[3]}$ verlaufen die Additionen von Carbenium-Ionen an Alkene streng regioselektiv, wobei die Orientierung durch die Stabilität der intermediären Carbenium-Ionen festgelegt wird ${ }^{[2,4]}$ (MarkownikowRegel). Dadurch unterscheiden sie sich von anderen elektrophilen Additionen wie Sulfenylierungen und Selenylierungen, bei denen die Orientierung durch den nucleophilen Angriff auf die verbrückte Zwischenstufe bestimmt wird (Schema 3).

Selbst das sterisch abgeschirmte Olefin 2 wird von Diarylcarbenium-Ionen 1 ausschließlich am tert-Butyl-substituierten Vinyl-Kohlenstoffatom angegriffen ${ }^{[6]}$, wodurch die Steuerung der Additionsrichtung durch elektronische Faktoren im intermediären Carbenium-Ion unterstrichen wird.

In gleicher Weise verläuft die Lewis-Säure-katalysierte Addition an Alkyl- und Aryl-substituierte Acetylene streng nach der Markownikow-Regel ${ }^{[7,8]}$. Alkyl-substituierte 1,3Diene reagieren so, daß Allyl-Kationen mit der maximalen Zahl terminaler Alkylgruppen gebildet werden, wodurch sich die in Schema 4 markierten Angriffspositionen ergeben $^{[4,6,9]}$. 1-Buten-3-in wird von C-Elektrophilen in 4-Position angegriffen, und die Umkehr der Additionsrichtung beim 1-Hexen-3-in (Schema 4) läßt sich wiederum durch den

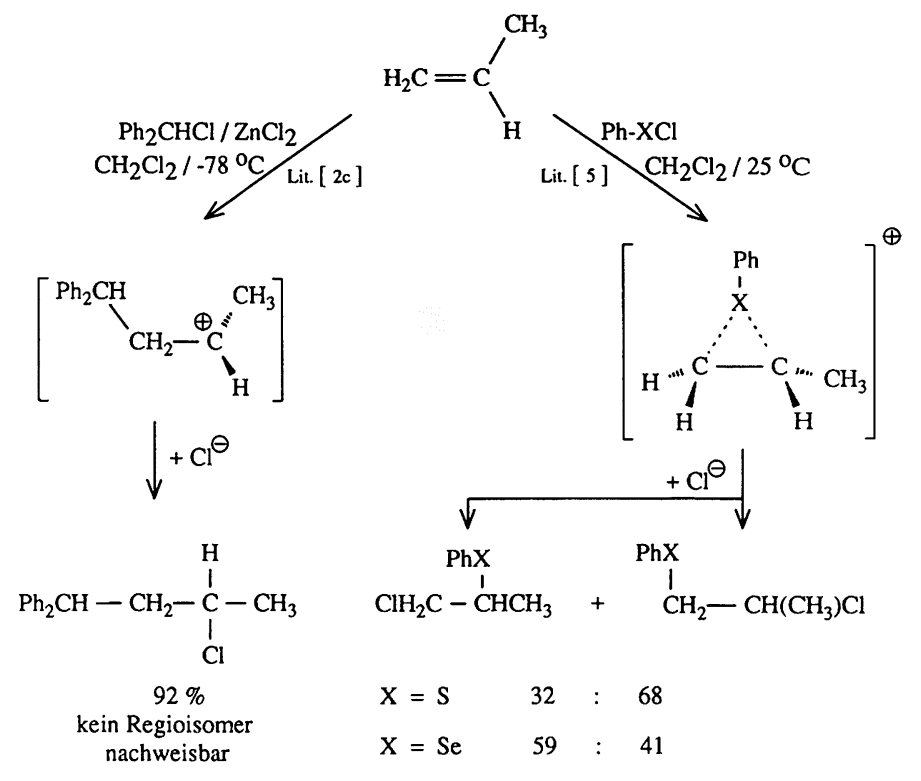

Schema 3.

elektronischen Einfluß der zusätzlichen Ethylgruppe auf die kationische Zwischenstufe erklären ${ }^{[2 \mathrm{~d}, 9]}$.

Nach MINDO/3-Rechnungen sollen Additionen von Carbenium-Ionen an Alkene über $\pi$-Komplexe verlaufen ${ }^{[10]}$,

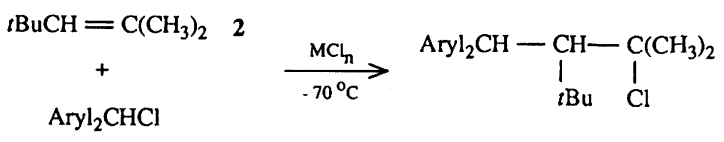

wodurch die hohe anti-Stereoselektivität bei carbokationischen Cyclisierungen ${ }^{[1 b, 11]}$ erklärt wurde. Stereochemische Untersuchungen von intermolekularen Additionen am Bei.

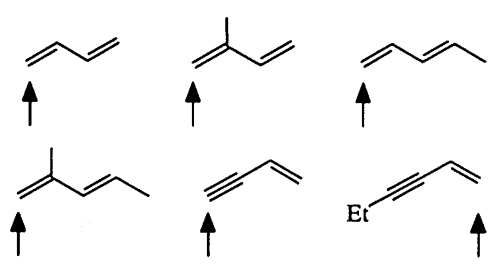

Schema 4. Positionen des Angriffs von Carbenium-Ionen an konjugierten Dienen und Eninen.

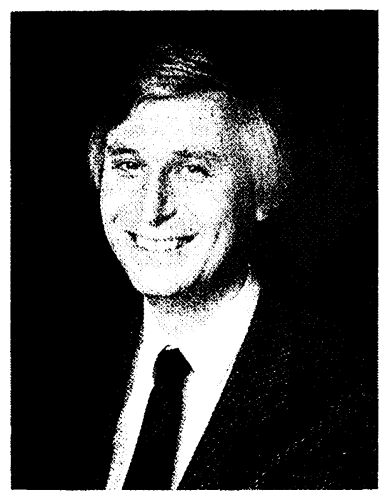

Herbert Mayr, geboren 1947 in Weilheim, Oberbayern, studierte Chemie an der Universität München und promovierte dort 1974 bei R. Huisgen. Nach einer einjährigen PostdoktorandenTätigkeit bei G. A. Olah an der Case-Western-Reserve-University in Cleveland/Ohio ging er 1976 zu P. von R. Schleyer in Erlangen, wo er sich 1980 habilitierte. 1984 übernahm er die Leitung des Instituts für Chemie an der Medizinischen Universität zu Lübeck, und 1990 erhielt er einen Ruf an die Technische Hochschule Darmstadt. Seine Arbeitsgebiete sind Synthesen über carbokationische Zwischenstufen, Kinetik von Ionen-Molekül-Reaktionen, lineare Freie-Energie-Beziehungen, carbokationische Telomerisationen und ungewöhnliche Cycloadditionen. 
spiel Lewis-Säure-katalysierter Umsetzungen von Diphenylmethylchloriden mit $(Z)$ und $(E)$-2-Buten zeigten, daß das Verhältnis von anti-zu syn-Additionsprodukten von der Art der Lewis-Säure abhängt, die Bildung des anti-Produkts 3 bzw. 4 jedoch jeweils stark begünstigt ist (Tabelle 1$)^{[12]}$
3

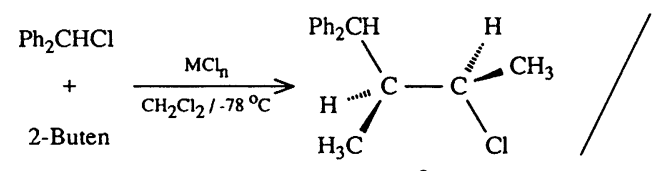<smiles>CCCCC(C)C(C)Cl</smiles>

Tabelle 1. Diastereomerenverhältnisse der Produkte der Lewis-Säure-katalysierten Umsetzungen von Diphenylmethylchlorid mit $(Z)$ - und $(E)$-2-Buten $\left(\mathrm{CH}_{2} \mathrm{Cl}_{2},-78^{\circ} \mathrm{C}\right)[\mathrm{a}]$.

\begin{tabular}{llc}
\hline Alken & $\mathrm{MCl}_{\mathrm{n}}$ & $\mathbf{3 : 4}$ \\
\hline (Z)-2-Buten & $\mathrm{ZnCl}_{2}$ & $86: 14$ \\
& $\mathrm{BCl}_{3}$ & $83: 17$ \\
& $\mathrm{SnCl}_{4}$ & $91: 9$ \\
& $\mathrm{TiCl}_{4}$ & $96: 4$ \\
$(E)$-2-Buten & $\mathrm{ZnCl}_{2}$ & $4: 96$ \\
& $\mathrm{BCl}_{3}$ & $12: 88$ \\
& $\mathrm{SnCl}_{4}$ & $8: 92$ \\
& $\mathrm{TiCl}_{4}$ & $<1:>99$ \\
\hline
\end{tabular}

[a] Die Reaktion liefert daneben Umlagerungsprodukte durch H-Verschiebungen (siehe Abschnitt 3).

Die in Tabelle 1 beschriebenen Befunde wurden durch das Auftreten partiell verbrückter Zwischenstufen 5 erklärt ${ }^{[12]}$. Als alternative Deutung dieser Beobachtungen wurde vorgeschlagen, daß eine Wechselwirkung zwischen dem Carbenium-Zentrum und einem benachbarten Arylring (siehe 6) die freie Rotation einschränkt ${ }^{[13]}$. Eine solche stabilisierende Wechselwirkung halten wir jedoch für wenig wahrscheinlich, da die Solvolyse von Alkylchloriden durch eine $\beta$-ständige Diphenylmethylgruppe sogar geringfügig verlangsamt wird $^{[14]}$.

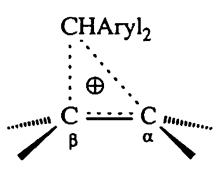

5

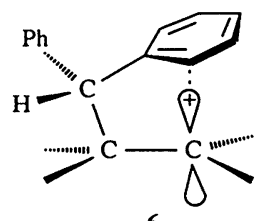

6<smiles>C[C@H](c1ccccc1)[C@H](Cl)c1ccccc1</smiles>

7
Mit zunehmendem Elektronenschub der Substituenten an $\mathrm{C}_{\alpha}$ verliert die Verbrückung in $\mathbf{5}$ an Bedeutung. Dementsprechend wird bei der Lewis-Säure-initiierten Umsetzung von Diphenylmethylchlorid mit beiden Isomeren des $\beta$-Methylstyrols als Hauptprodukt das Diastereomer 7 gebildet, wodurch eine weitgehend freie Rotation in der Zwischenstufe angezeigt wird. Entsprechende Veränderungen der Stereoselektivität wurden bei Bromierungen von Butenen und Styrolen beobachtet ${ }^{[15]}$.

Die stereochemischen Befunde bei Lewis-Säure-induzierten Additionen von Alkylchloriden an Alkine lassen sich dagegen durch nichtverbrückte Zwischenstufen erklären ${ }^{[8]}$. Die Angriffsrichtung eines Nucleophils am intermediären Vinylkation 8 wird durch die relative Größe der Substituenten $\mathrm{R}$ und $\mathrm{R}^{\prime}$ festgelegt (Schema 5).

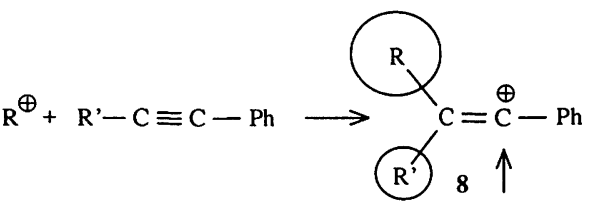

Schema 5 .

Diese Deutung wurde durch die Isolierung eines 50:50Gemisches bei der Benzylierung von 1,3-Diphenylpropin mit markiertem Benzylchlorid erhärtet ${ }^{[8]}$ (Schema 6).

$$
\begin{aligned}
& \mathrm{Ph}-\mathrm{CH}_{2}-\mathrm{C} \equiv \mathrm{C}-\mathrm{Ph} \\
& \text { Schema } 6 .
\end{aligned}
$$

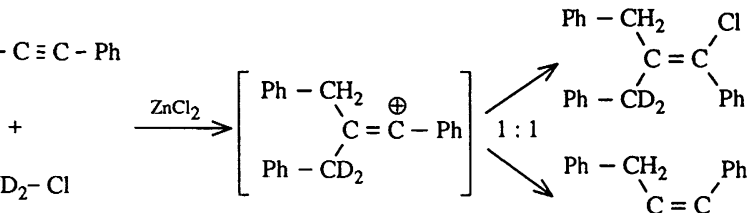

$$
\begin{aligned}
& \stackrel{\mathrm{C}}{\mathrm{C}}=\mathrm{C}^{\prime}
\end{aligned}
$$

Über die Diastereoselektivität des CC-Verknüpfungsschritts beim elektrophilen Angriff von Carbenium-Ionen auf Alkene gibt es kaum Untersuchungen. Im allgemeinen fanden wir nur geringe Stereoselektivitäten, wie das Beispiel der Lewis-Säure-katalysierten Addition von 4-Chlor-2-penten (,,1,3-Dimethylallylchlorid") an (Z)-2-Buten illustriert. Der si,re-Übergangszustand 9 ist gegenüber dem si,si- (und re,re-)Übergangzustand $\mathbf{1 0}$ nur geringfügig begünstigt

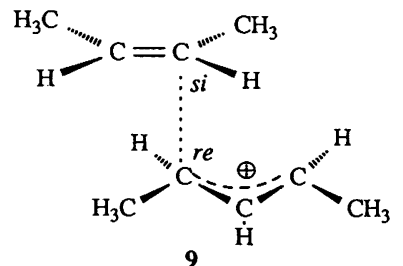<smiles>C/C=C\[14CH2][14CH](C)/C=C\C</smiles>

10
(75:25), und im Fall der entsprechenden Addition an (E)-2Buten sinkt der Vorzug von si,re gegenüber si,si (und re,re) auf $57: 43^{[16]}$.

\section{Umlagerungen}

1,2-Alkyl- und Wasserstoffverschiebungen zählen zu den schnellsten Reaktionen der Organischen Chemie ${ }^{[1]}$. Beim Studium persistenter Carbokationen lassen sich entartete 1,2-Wanderungen auf der NMR-Zeitskala häufig nicht einmal bei $-120^{\circ} \mathrm{C}$ ausfrieren, so daß spezielle Techniken entwickelt worden sind, um die Grundzustandsstruktur derartiger Kationen zu ermitteln ${ }^{[17 a, b]}$. Für entartete 1,2-Wasserstoff- und Methylverschiebungen bei tertiären CarbeniumIonen beträgt die Barriere üblicherweise $12-15 \mathrm{~kJ} \mathrm{~mol}^{-1}$; sie wird kleiner, wenn die Umlagerung mit einer Stabilisierung des Carbenium-Ions verbunden ist. Trotz dieser niedrigen Barrieren spielen Umlagerungen der nach Schema 2 intermediär auftretenden Carbenium-Ionen häufig eine erstaunlich geringe Rolle. Obwohl 1,2-H-Verschiebungen, bei denen sekundäre Carbenium-Ionen in tertiäre umlagern, kei- 
ne Aktivierungsbarriere haben ${ }^{[17]}$, erhält man bei der Umsetzung von Diphenylmethylchlorid mit den $(E, Z)$-isomeren 2-Butenen Gemische aus den regulären Additionsprodukten 3 und $\mathbf{4}$ sowie den von 11 abgeleiteten Umlagerungsprodukten 13 (Schema 7) ${ }^{[12]}$. Wegen der partiellen Verbrückung von $12 \mathrm{muß}$ der 1,2-Wanderung eine Rotation um die ehemalige CC-Doppelbindung vorausgehen, so daß die Reaktion von 12 mit $\mathrm{MCl}_{\mathrm{n}+1}^{\ominus}$ (unter Bildung von 3 und 4) mit der Umlagerung zu 11 konkurrieren kann. So entstehen aus Diphenylmethylchlorid und $(E)$-2-Buten überwiegend die regulären Additionsprodukte 3 und 4 (Tabelle 1), und nur $20 \%$ der Reaktionsprodukte leiten sich vom umgelagerten Kation 11 $\mathrm{ab}$, wenn $\mathrm{ZnCl}_{2} \cdot \mathrm{OEt}_{2}, \mathrm{BCl}_{3}$ oder $\mathrm{SnCl}_{4}$ als $\mathrm{Katalysatoren}$ verwendet werden $\left(\mathrm{CH}_{2} \mathrm{Cl}_{2},-78^{\circ} \mathrm{C}\right)$. $\mathrm{Mit} \mathrm{TiCl}_{4}$ läßt sich die Umlagerung sogar auf $4 \%$ zurückdrängen. Die entsprechende Reaktion mit $(Z)$-2-Buten liefert einen höheren Anteil an umgelagerten Produkten (bis $65 \%$ ), der wiederum bei Verwendung von $\mathrm{TiCl}_{4}$ am geringsten ist $(21 \%)^{[12]}$.

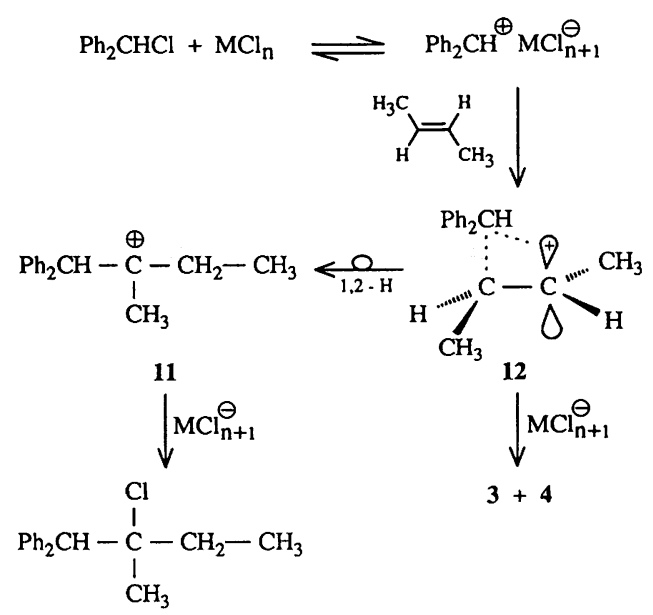

Schema 7

Da beim elektrophilen Angriff auf 1,2-Dialkyl-substituierte Ethylene stets sekundäre Carbenium-Ionen entstehen, die durch 1,2-H-Verschiebung in tertiäre CarbeniumIonen übergehen können, erhält man auch bei Umsetzungen von Cyclopenten und Cyclohexen stets beträchtliche Mengen an Umlagerungsprodukten ${ }^{[18]}$. Angesichts dieser Befunde ist es überraschend, daß bei der Aluminiumchlorid-katalysierten Umsetzung von tert-Butylchlorid mit Ethylen keine Umlagerung auftritt und ausschließlich das Additionsprodukt 14 erhalten wurde ${ }^{[19]}$. Der Verlauf über ein primäres Carbenium-Ion muß daher ausgeschlossen werden, und die Entstehung von 14 kann über ein verbrücktes Carbokation (tert-Butyl-überbrücktes Ethylen) gedeutet werden. Alternativ könnte man annehmen, daß wegen der geringen Stabilität des 3,3-Dimethyl-1-butyl-Kations der Angriff des tert-Butyl-Kations an Ethylen durch das $\mathrm{AlCl}_{4}^{\ominus}$-Ion nucleophil unterstützt wird (Schema 8) ${ }^{[20]}$.

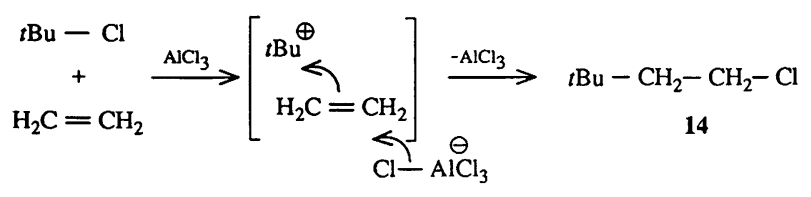

Schema 8
Umlagerungen, die mit keiner oder nur einer geringfügigen Erhöhung der Stabilität des Carbenium-Ions verbunden sind (z. B. tertiär $\rightarrow$ tertiär oder sekundär $\rightarrow$ sekundär) werden gelegentlich beobachtet, doch lassen sie sich im allgemeinen zurückdrängen oder völlig unterdrücken, indem die Geschwindigkeit der Ionenkombination $\left(v_{1}\right.$, Schema 9) erhöht wird.

$$
\begin{aligned}
& \mathrm{R}^{\oplus}+\mathrm{MX}_{\mathrm{n}+1}^{\ominus} \stackrel{K_{\mathrm{D}}}{\longrightarrow}\left[\mathrm{R}^{\oplus} \mathrm{MX_{ \textrm {n } + 1 } ^ { \ominus }}\right] \stackrel{v_{1}}{\longrightarrow} \mathrm{R} \cdot \mathrm{X}+\mathrm{MX}_{\mathrm{n}} \\
& v_{-2} \uparrow \downarrow v_{2} \quad \text { Ionenpa } \\
& \mathrm{R}^{, \oplus}+\mathrm{MX}_{\mathrm{n}+1}^{\ominus} \stackrel{K_{\mathrm{D}}}{\longleftarrow}\left[\mathrm{R}^{,}{ }^{\oplus} \mathrm{M} \mathrm{X}_{\mathrm{n}+1}^{\ominus}\right] \stackrel{v_{4}}{\longrightarrow} \mathrm{R}^{\prime}-\mathrm{X}+\mathrm{MX}_{\mathrm{n}}
\end{aligned}
$$

Schema 9.

Da Umlagerungen $\left(\mathrm{R}^{\oplus} \rightarrow \mathrm{R}^{\prime}\right)$ auf der Stufe gepaarter wie ungepaarter Ionen stattfinden können, hängt ihre Geschwindigkeit $\left(v_{2}+v_{3}\right)$ nicht $\left(v_{2}=v_{3}\right)$ oder nur wenig $\left(v_{2} \approx v_{3}\right)$ vom Ausmaß der Ionenpaarung ab. Dagegen ist die Geschwindigkeit der Ionenkombination $\left(v_{1}, v_{4}\right)$ der Konzentration an Ionenpaaren annähernd proportional und kann beispielsweise durch Zusatz eines quartären AmmoniumSalzes $\left(\mathrm{R}_{4} \mathrm{NMX}_{\mathrm{n}}\right)$ beschleunigt werden. Tabelle 2 zeigt, wie mit zunehmender $\mathrm{BCl}_{4}^{\ominus}$-Konzentration die Ausbeute an 17 gegenüber der des Cyclisierungsprodukts 18 ansteigt. Das Verhältnis 17:18 bleibt jedoch annähernd konstant, sobald der überwiegende Teil der Ionen gepaart vorliegt ${ }^{[14,21]}$.

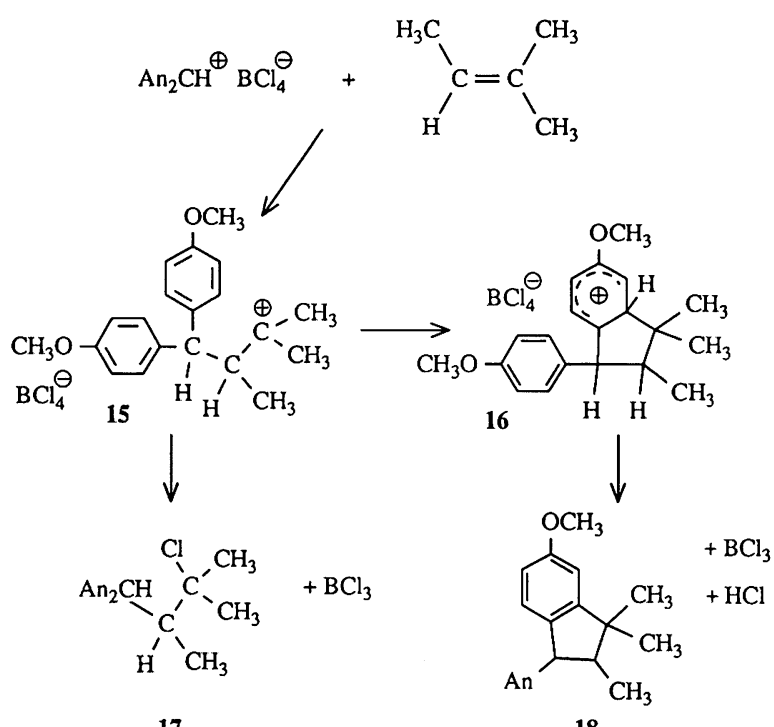

17

18

Tabelle 2. Der Einfluß von Benzyltriethylammonium-tetrachloroborat auf das Produktverhältnis bei der Umsetzung von (Bis(p-anisyl)carbenium-tetrachloroborat mit 2-Methyl-2-buten in $\mathrm{CH}_{2} \mathrm{Cl}_{2}$ bei $-70^{\circ} \mathrm{C}[14,21]$

\begin{tabular}{llcl}
\hline $\begin{array}{l}c\left(\mathrm{An}_{2} \mathrm{CH}^{\oplus}\right) \\
{\left[\mathrm{mol} \mathrm{L}^{-1}\right][\mathrm{a}]}\end{array}$ & $\begin{array}{l}c\left(\mathrm{BCl}_{4}^{\ominus}\right) \\
{\left[\mathrm{mol} \mathrm{L}^{-1}\right][\mathrm{a}, \mathrm{b}]}\end{array}$ & $\begin{array}{c}\text { Ionen } \\
\text { gepaart:ungepaart }\end{array}$ & $\begin{array}{l}\text { Produktverhältnis } \\
17: 18\end{array}$ \\
\hline $5.5 \times 10^{-5}$ & $5.5 \times 10^{-5}$ & $17: 83$ & $41: 59$ \\
$5.5 \times 10^{-5}$ & $5.6 \times 10^{-4}$ & $54: 46$ & $68: 32$ \\
$5.5 \times 10^{-5}$ & $3.4 \times 10^{-3}$ & $78: 22$ & $82: 18$ \\
$5.5 \times 10^{-5}$ & $1.5 \times 10^{-2}$ & $89: 11$ & $86: 14$ \\
$5.5 \times 10^{-5}$ & $4.9 \times 10^{-2}$ & $94: 6$ & $84: 16$ \\
\hline
\end{tabular}

[a] Anfangskonzentration. [b] Summe aus $c\left(\mathrm{An}_{2} \mathrm{CH}^{\oplus} \mathrm{BCl}_{4}^{\ominus}\right)$ und $c\left(\mathrm{BzNEt}_{3}^{\oplus} \mathrm{BCl}_{4}^{\ominus}\right)$. 
In gleicher Weise läßt es sich erklären, daß die $\mathrm{ZnCl}_{2}$-katalysierte Umsetzung des Allylchlorids 19 mit Styrol ein 65:35Gemisch aus 23 und 24 liefert, während in Gegenwart von Benzyltriethylammonium-trichlorozinkat unter sonst gleichen Bedingungen reines 23 erhalten wird ${ }^{[4 b, 22]}$. Die Umlagerung $\mathbf{2 0} \rightarrow \mathbf{2 1} \rightarrow \mathbf{2 2}$ läßt sich somit unterdrücken, wenn durch Erhöhung der $\mathrm{ZnCl}_{3}^{\ominus}$-Konzentration die Übertragung eines Chlorid-Ions auf 20 beschleunigt wird (Schema 10).

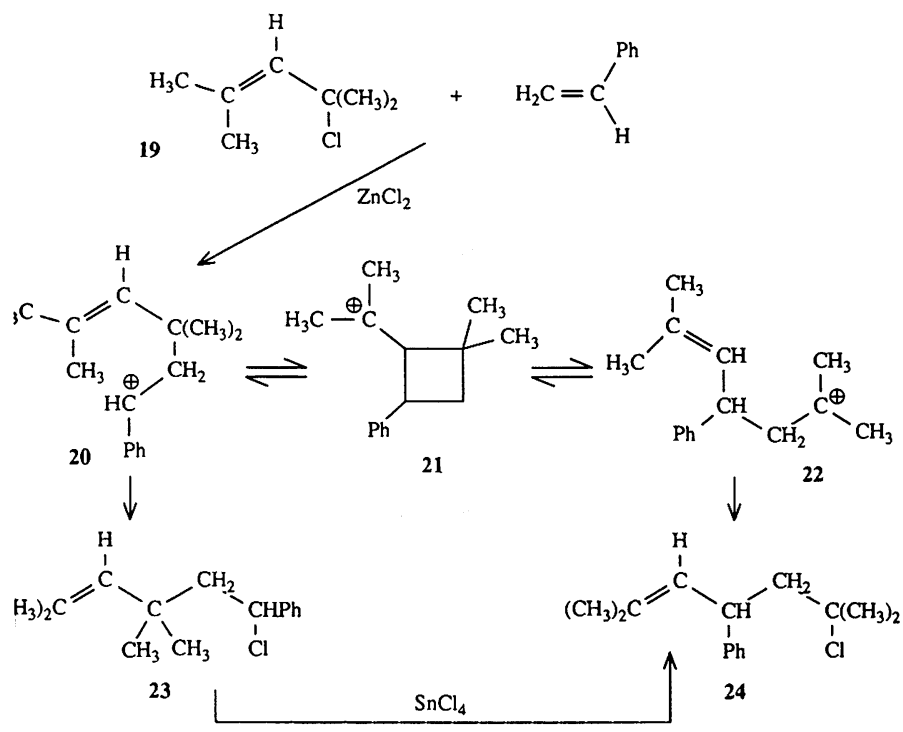

Schema 10

In erster Näherung kann man davon ausgehen, daß auch durch Verwendung einer möglichst schwachen Lewis-Säure Umlagerungen zurückgedrängt werden, weil eine Verringerung der Stabilität von $\mathrm{MX}_{\mathrm{n}+1}^{\ominus}$ die Übertragung eines Halogenid-Ions $\mathrm{X}^{\ominus}$ auf ein Carbenium-Ion erleichtert. Dennoch besteht kein strenger Zusammenhang zwischen Umlagerungstendenz und thermodynamischer Stabilität von $\mathrm{MX}_{\mathrm{n}+1}^{\ominus}$, wie die oben erwähnte Umsetzung von Benzhydrylchlorid mit den 2-Butenen zeigt: Obwohl $\mathrm{TiCl}_{4}$ ein stärkerer Chlorid-Acceptor als $\mathrm{ZnCl}_{2} \cdot \mathrm{OEt}_{2}$ ist, wurde bei der Reaktion mit $\mathrm{TiCl}_{4}$ ein geringerer Prozentsatz an umgelagerten Produkten beobachtet. Keine Möglichkeit zur Unterdrückung von Umlagerungen fanden wir, wenn durch den elektrophilen Angriff Kationen des Cyclopropylcarbinyl-, Cyclopropyl- oder Norbornyl-Typs entstanden; die Alkylierung von (1-Methylethenyl)cyclopropan ${ }^{[23]}$, Methylencyclopropan ${ }^{[24]}$ und Norbornen ${ }^{[25]}$ lieferte ausschließlich die umgelagerten Produkte 25-27.

$$
\begin{aligned}
& \begin{array}{cc}
\stackrel{\mathrm{CH}_{3}}{\mathrm{R}}-\mathrm{CH}_{2}-\stackrel{\mathrm{CH}^{\mathrm{C}}=\mathrm{CH}}{=}-\mathrm{CH}_{2}-\mathrm{CH}_{2}-\mathrm{Cl} & \mathrm{R}-\mathrm{CH}_{2}-\stackrel{!}{\mathrm{C}}=\mathrm{CH}_{2}
\end{array} \\
& 25 \\
& 26
\end{aligned}
$$

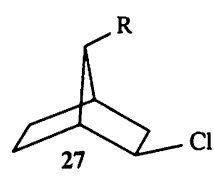

\section{Thermodynamik der Additionsreaktion: Addition versus Grob-Fragmentierung}

Für die thermodynamischen Überlegungen betrachten wir vier Grenzfälle, die sich hinsichtlich des Ionisationsgrads von Reaktanten und Produkten unterscheiden: a) Reaktanten und Produkte kovalent

b) Reaktanten und Produkte ionisch

c) Reaktanten kovalent, Produkte ionisch

d) Reaktanten ionisch, Produkte kovalent

Die hier gewählte Untergliederung $(\mathrm{a}-\mathrm{d})$ findet sich in den Subskripten $\left(\Delta G_{\mathrm{a}}^{0} \ldots \Delta G_{\mathrm{d}}^{0}\right)$ in Schema 11 wieder.

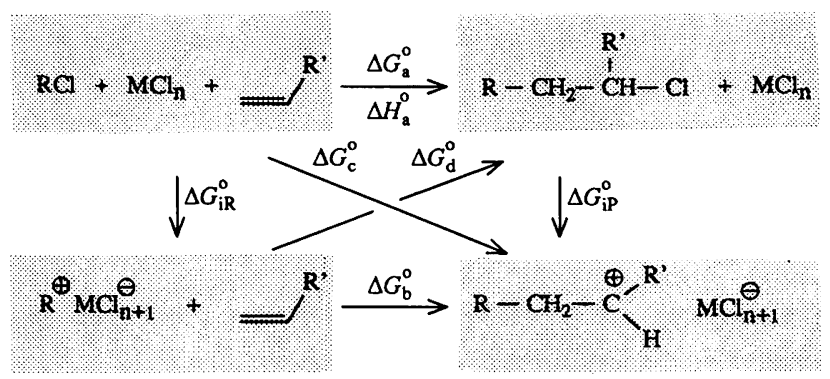

Schema 11.

Fall a: Treten Carbenium-Ionen nur in kleinen Gleichgewichtskonzentration auf, d. h. liegen die Reaktanten und die Produkte weitgehend kovalent vor, ergibt sich $\Delta H_{\mathrm{a}}^{0}$ aus dem Energiegewinn der Umwandlung einer $\pi_{\mathrm{CC}^{-}}$in eine $\sigma_{\mathrm{CC}}$-Bindung. Aus den gemittelten Bindungsenergien ${ }^{[26]}$ schätzt man hierfür einen Betrag von $\Delta H_{\mathrm{a}}^{0}=-84 \mathrm{~kJ} \mathrm{~mol}^{-1} \mathrm{ab}$, der die kalorimetrisch bestimmten Reaktionswärmen ${ }^{[27]}$ der Additionen von Benzhydrylchloriden an 2-Methyl-1-penten (-84.5), Styrol (-83.4), 2-Methyl-2-buten ( -78.9$)$ und Isopren $(-74.2)$ annähernd wiedergibt. Die unterschiedlichen Grundzustände der $\pi$-Systeme sowie die unterschiedlichen sterischen Spannungen in den Additionsprodukten beeinflussen somit die Größenordnung der Additionsenthalpie nur wenig. Mit der aus Inkrementen ${ }^{[28]}$ ermittelten Reaktionsentropie $\left(-150\right.$ bis $\left.-175 \mathrm{~J} \mathrm{~mol}^{-1} \mathrm{~K}^{-1}\right)$ ergibt sich $\Delta G_{\mathrm{a}}^{0}\left(-70^{\circ} \mathrm{C}\right) \mathrm{zu}$ etwa $-50 \mathrm{~kJ} \mathrm{~mol}^{-1}$. Das von der Art der Lewis-Säure unabhängige $\Delta G_{\mathrm{a}}^{0}$ erfährt nur dann merkliche Veränderungen, wenn in den Additionsprodukten beträchtliche sterische Spannungen auftreten. Da aber selbst das sperrige Tris( $p$-chlorphenyl)methylchlorid 28 bei $-10^{\circ} \mathrm{C}$ mit Isobuten glatt unter Bildung des Addukts 29 reagiert $^{[29]}$, ist zu folgern, daß $\Delta G_{\mathrm{a}}^{0}$ nur in Extremfällen positive Werte annimmt.

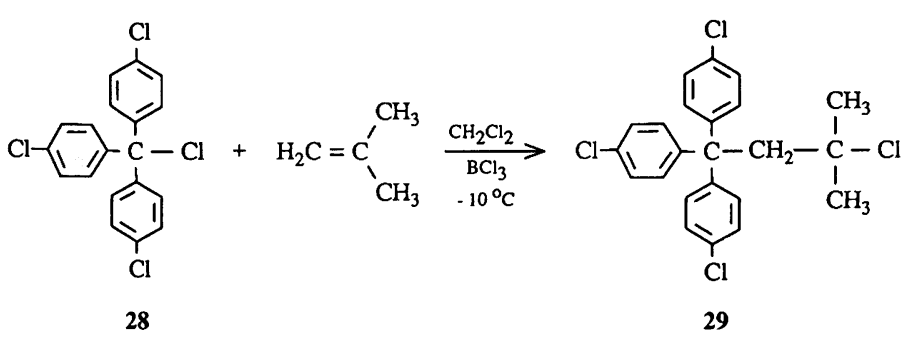

Fall b: Liegen die Reaktanten und Produkte ionisch vor, ergibt sich die Freie Standardreaktionsenthalpie entsprechend Schema 11 nach Gleichung (a).

$\Delta G_{\mathrm{b}}^{0}=\Delta G_{\mathrm{a}}^{0}-\left(\Delta G_{\mathrm{iR}}^{0}-\Delta G_{\mathrm{iP}}^{0}\right)$

Gleichung (a) zeigt, da $\triangle G_{\mathrm{a}}^{0}$ nun um den Unterschied der freien Ionisationsenthalpie der Produkte und der Reaktan- 
ten korrigiert werden muß. Wir diskutierten früher, daß im Fall ionischer Reaktanten und Produkte die selektive Bildung von 1:1-Produkten nur möglich ist, wenn $\Delta G_{\mathrm{iR}}^{0}>$ $\Delta G_{\mathrm{iP}}^{0}$, d. h. wenn $\mathrm{R}^{\oplus}$ weniger stabilisiert ist als das daraus entstehende Carbenium-Ion R- $\mathrm{CH}_{2}-{ }^{\oplus} \mathrm{CHR}^{\prime}$ (addition rate control); andernfalls bilden sich Polymere ${ }^{\left[2 \mathrm{a},{ }^{30]}\right.}$. Da nach obigen Ausführungen $\Delta G_{\mathrm{a}}^{0}$ üblicherweise negativ ist, muß dies unter der Voraussetzung $\Delta G_{\mathrm{iR}}^{0}>\Delta G_{\mathrm{iP}}^{0}$ auch für $\Delta G_{\mathrm{b}}^{0}$ gelten. Mit anderen Worten: Die Addition eines wenig stabilisierten Carbenium-Ions an eine CC-Doppelbindung, die zur Bildung eines besser stabilisierten Carbenium-Ions führt, ist bei den üblichen Reaktionstemperaturen immer exergon.

Fall c: Die Bildung ionischer Produkte aus kovalenten Reaktanten ist gegeben, falls $\Delta G_{\mathrm{iR}}^{0}>0$ und $\Delta G_{\mathrm{iP}}^{0}<0$. Da nunmehr $\Delta G_{\mathrm{a}}^{0}$ und $\Delta G_{\mathrm{iP}}^{0}$ negativ sind, muß dies auch für $\Delta G_{\mathrm{c}}^{0}$ gelten [Gl. (b)].

$\Delta G_{\mathrm{c}}^{0}=\Delta G_{\mathrm{a}}^{0}+\Delta G_{\mathrm{ip}}^{0}$

Es ist somit zu erwarten, daß thermodynamische Gründe einer Additionsreaktion unter den Bedingungen $c$ nicht im Wege stehen können.

Fall d: Sollen aus ionischen Reaktanten kovalente Produkte entstehen (d.h. $\Delta G_{\mathrm{iR}}^{0}<0$ und $\Delta G_{\mathrm{iP}}^{0}>0$ ), ergibt sich die Freie Standardreaktionsenthalpie der Addition nach Gleichung (c).

$\Delta G_{\mathrm{d}}^{0}=\Delta G_{\mathrm{a}}^{0}-\Delta G_{\mathrm{iR}}^{0}$

Nach Gleichung (c) wird $\Delta G_{\mathrm{d}}^{0}$ negativ, wenn $\Delta G_{\mathrm{a}}^{0}<\Delta G_{\mathrm{i} \mathbf{R}}^{0}$. Für $\Delta G_{\mathrm{a}}^{0}\left(-70^{\circ} \mathrm{C}\right)$ wurde oben ein ungefährer Wert von - $50 \mathrm{~kJ} \mathrm{~mol}^{-1}$ abgeleitet, so daß Carbenium-Salze sich nur dann unter Bildung kovalenter Addukte an Alkene addieren können, wenn $\Delta G_{\mathrm{iR}}^{0} \gtrsim-50 \mathrm{~kJ} \mathrm{~mol}^{-1}$. Da $\Delta G_{\mathrm{iR}}^{0}$ von der Struktur von $\mathrm{R}^{\oplus}$ wie auch von der Lewis-Acidität von $\mathrm{MCl}_{\mathrm{n}}$ abhängt, ergibt sich, daß im Fall d die thermodynamische Triebkraft zunimmt, wenn die Stabilisierung von $\mathrm{R}^{\oplus}$ oder die Affinität der Lewis-Säure $\mathrm{MCl}_{\mathrm{n}} \mathrm{zu}$ Chlorid-Ionen vermindert wird.

Für die Ionisation von Diarylmethylchloriden mit $\mathrm{BCl}_{3}$ $\left(\mathrm{CH}_{2} \mathrm{Cl}_{2},-70^{\circ} \mathrm{C}\right)$ läßt sich die Korrelation (d) herleiten ${ }^{[31]}$.

$\Delta G_{\mathrm{iR}}^{0} / \mathrm{kJ} \mathrm{mol}^{-1}=-6.1 \mathrm{p} K_{\mathrm{R} \oplus}-67.1$

Setzt man den Ausdruck (d) sowie $\Delta G_{\mathrm{a}}^{0} \approx-50 \mathrm{~kJ} \mathrm{~mol}^{-1}$ in Gleichung (c) ein, erhält man Gleichung (e).

$\Delta G_{\mathrm{d}}^{0} / \mathrm{kJ} \mathrm{mol}^{-1} \approx 6.1 \mathrm{p} K_{\mathrm{R} \odot}+17.1$

Daraus geht hervor, daß für die Reaktion von Carbeniumtetrachloroboraten mit Alkenen unter Bildung kovalenter Produkte $\left(\mathrm{CH}_{2} \mathrm{Cl}_{2},-70^{\circ} \mathrm{C}\right)$ ein positiver Wert von $\Delta G_{\mathrm{d}}^{0}$ resultiert, wenn Carbenium-Ionen mit $\mathrm{p} K_{\mathrm{R} \circledast} \gtrsim-2.8$ eingesetzt werden ${ }^{[33]}$. Sollen besser stabilisierte Carbenium-Ionen $\left(\mathrm{p} K_{\mathrm{R} \oplus} \gtrsim-2.8\right)$ mit Alkenen zur Reaktion gebracht werden $\left(\mathrm{CH}_{2} \mathrm{Cl}_{2},-70^{\circ} \mathrm{C}\right)$, muß $\mathrm{BCl}_{4}^{\ominus}$ durch ein Anion mit höherer Lewis-Basizität ersetzt werden.

Die Umkehr der hier diskutierten Additionsreaktionen ist die Grob-Fragmentierung ${ }^{[32]}$. In qualitativer Übereinstimmung mit Gleichung (e) wird die Fragmentierung kovalenter Produkte dann beobachtet, wenn bei dem Zerfall ein gut stabilisiertes Carbenium-Ion (großer $\mathrm{p} K_{\mathrm{R} \oplus}$-Wert) gebildet

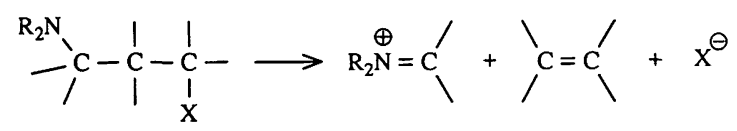

Schema 12

wird, wie dies beispielsweise bei der Ionisation $\beta$-Amino-substituierter Alkylderivate der Fall ist (Schema 12).

Fragmentierung unter Bildung des aromatischen Tropylium-Ions $31\left(\mathrm{p} K_{\mathrm{R} \oplus}=+4.76\right)$ beobachtete man beim Behandeln von $30(\mathrm{R}=\mathrm{H})$ mit Schwefelsäure ${ }^{[34]}$. Um den oben diskutierten Gegenionen-Effekt auf die Thermodynamik des

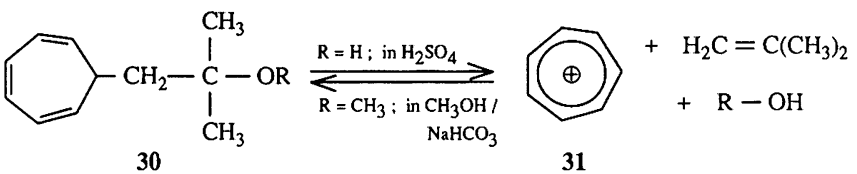

Fragmentierungs-Additions-Gleichgewichts $\mathrm{zu}$ illustrieren, setzten wir Tropylium-tetrafluoroborat $\left(31 \cdot \mathrm{BF}_{4}^{\ominus}\right)$ in Methanol mit Isobuten um und isolierten $30\left(\mathrm{R}=\mathrm{CH}_{3}\right)$ in $45 \%$ Ausbeute ${ }^{[35]}$.

\section{Kinetische Untersuchungen}

\subsection{Methoden}

Zusammenhänge zwischen Struktur und Reaktionsgeschwindigkeit wurden durch Konkurrenzexperimente sowie durch Messungen absoluter Geschwindigkeitskonstanten ermittelt. Da die Reaktionsgeschwindigkeit der CarbeniumIonen von der Natur der Gegenionen unabhängig ist (siehe Abschnitt 5.1.2), lassen sich die beiden Meßreihen direkt miteinander verknüpfen.

\subsubsection{Bestimmung relativer Reaktivitäten durch Konkurrenzexperimente}

Relative Alken-Reaktivitäten bestimmte man, indem Diarylcarbenium-Ionen in situ aus Diarylmethylchloriden und Lewis-Säuren in Gegenwart eines Überschusses zweier konkurrierender Olefine erzeugt wurden ${ }^{[23,36]}$. Arbeitet man hierbei unter Bedingungen, unter denen Folgereaktionen der 1:1-Produkte mit den im Überschuß vorliegenden Alkenen ausgeschlossen sind, läßt sich das Reaktivitätsverhältnis der beiden Alkene aus dem Verhältnis der Additionsprodukte 32 und 33 ermitteln (Schema 13).

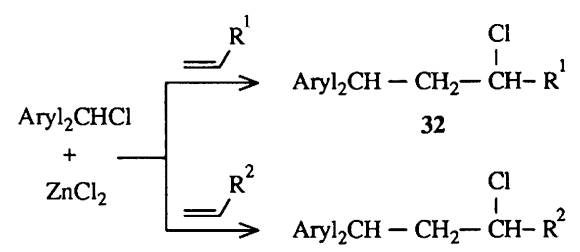

33 
Entsprechend wurden relative Elektrophil-Reaktivitäten aus dem Verhältnis der 1:1-Addukte 34 und 35 ermittelt, die bei Zugabe eines Alkens zu einem Gemisch von Elektrophilen entstehen (Schema 14) ${ }^{[30,37]}$. Wie in Abschnitt 5.2

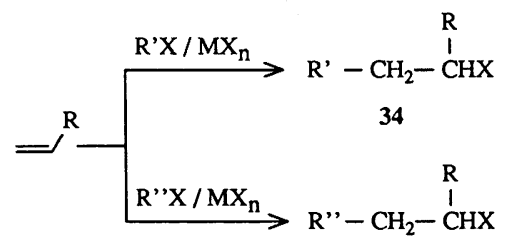

35

Schema 14

gezeigt wird, ist die relative Elektrophilie stark abhängig von Art und Menge der Lewis-Säure $M_{n}$.

\subsubsection{Bestimmung absoluter Geschwindigkeitskonstanten}

Absolute Geschwindigkeitskonstanten für die Reaktionen von Carbenium-Ionen mit Alkenen wurden erstmals dadurch bestimmt, daß die Extinktionsabnahme pulsradiolytisch erzeugter ${ }^{[38]}$ Benzhydryl- und Benzyl-Kationen in 1,2Dichlorethan in Gegenwart von Alkenen und Dienen verfolgt wurde ${ }^{[39]}$. Dieses Verfahren eignet sich jedoch nur zur Untersuchung sehr rascher Reaktionen $\left(k_{2}>10^{5}\right.$ bis $10^{6} \mathrm{~L} \mathrm{~mol}^{-1} \mathrm{~s}^{-1}$ ).

Ein wesentlich erweiterter Datensatz basiert auf dem Befund, daß sich zahlreiche Diarylmethylchloride durch LewisSäure in Dichlormethan vollständig ionisieren lassen und dabei farbige, elektrisch leitende Lösungen ergeben ${ }^{[40]}$. Setzt man diesen Lösungen Alkene zu, kommt es häufig zur Bildung kovalenter Produkte (Schema 15), und die Reaktions-

$$
\begin{aligned}
& \text { Aryl }_{2} \mathrm{CH}^{\oplus} \mathrm{MX} \mathrm{X}_{\mathrm{n}+1}^{\ominus} \quad \text { ionisch } \\
& +=^{\mathrm{R}} \downarrow^{\text {langsam }} \\
& {\left[\text { Aryl }_{2} \mathrm{CH}-\mathrm{CH}_{2}-\stackrel{\oplus}{\mathrm{CHR}} \quad \mathrm{MX}_{\mathrm{n}+1}^{\ominus}\right]} \\
& \downarrow \text { rasch }
\end{aligned}
$$

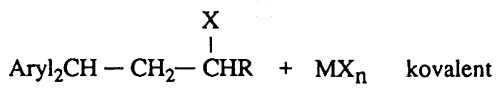

Schema 15

geschwindigkeit läßt sich photometrisch und konduktometrisch verfolgen ${ }^{[41,14]}$. Dabei handelt es sich um Reaktionen 2. Ordnung [Gl. (f)], wobei $\left[\mathrm{Aryl}_{2} \mathrm{CH}^{\oplus}\right]$ der Summe der Konzentrationen ungepaarter und gepaarter DiarylcarbeniumIonen entspricht.

$$
-\mathrm{d}\left[\mathrm{Aryl}_{2} \mathrm{CH}^{\oplus}\right] / \mathrm{d} t=k_{2}\left[\operatorname{Aryl}_{2} \mathrm{CH}^{\oplus}\right][\text { Alken }]
$$

Da im Verlauf einer Reaktion die Ionen-Konzentration sinkt, so daß das Verhältnis ungepaarter/gepaarter Ionen ansteigt, impliziert die Gültigkeit von Gleichung (f), daß ungepaarte und mit komplexen Gegenionen gepaarte Di-
arylcarbenium-Ionen die gleiche Reaktivität gegenüber Alkenen besitzen. In Einklang mit dieser Schlußfolgerung wird die Reaktionsgeschwindigkeit durch den Zusatz von quartären Ammoniumsalzen mit dem gleichen Gegenion nicht verändert.

Eine Erklärung dieser Beobachtung ergibt sich aus dem Befund, daß im Gegensatz zur Ionisationskonstanten $K_{\mathrm{I}}$ (Schema 16) die Dissoziationskonstante $K_{\mathrm{D}}$ von Salzen mit

$$
\mathrm{RX}+\mathrm{MX}_{\mathrm{n}} \stackrel{K_{\mathrm{I}}}{\rightleftharpoons} \mathrm{R}_{\substack{\text { Ionenpaar } \\ \mathrm{MX}_{\mathrm{n}+1}}}^{\stackrel{K_{\mathrm{D}}}{\rightleftharpoons}} \mathrm{R}_{\substack{\text { ungepaarte } \\ \text { Ionen }}}^{\oplus}+\mathrm{MX}_{\mathrm{n}+1}^{\ominus}
$$

Schema 16.

komplexen Anionen weitgehend unabhängig von der Natur der Ionen ist. Für alle uns bekannten Kombinationen der Kationen $\mathrm{Aryl}_{2} \mathrm{CH}^{\oplus}, \mathrm{Aryl}_{3} \mathrm{C}^{\oplus}$, Tropylium, Pyrylium und Tetraethylammonium mit den Anionen $\mathrm{BCl}_{4}^{\ominus}, \mathrm{SbCl}_{6}^{\ominus}, \mathrm{SbF}_{6}^{\ominus}$ und $\mathrm{AsF}_{6}^{\ominus}$ wurden in Dichlormethan $K_{\mathrm{D}}$-Werte von etwa $10^{-4} \mathrm{~mol} \mathrm{~L}^{-1}$ bestimmt ${ }^{[40]}$. Es ist daher anzunehmen, daß $K_{\mathrm{D}}$ auch für Reaktanten, Produkte und aktivierte Komplexe der in Abbildung 1 skizzierten Reaktionsfolge den gleichen Wert hat, woraus ein konstantes Populationsverhältnis zwischen gepaarten und ungepaarten Ionen in Grund- und Übergangszustand resultiert. Die Unabhängigkeit der Reaktionsgeschwindigkeit vom Ausmaß der Ionenpaarung wird dadurch erklärbar.

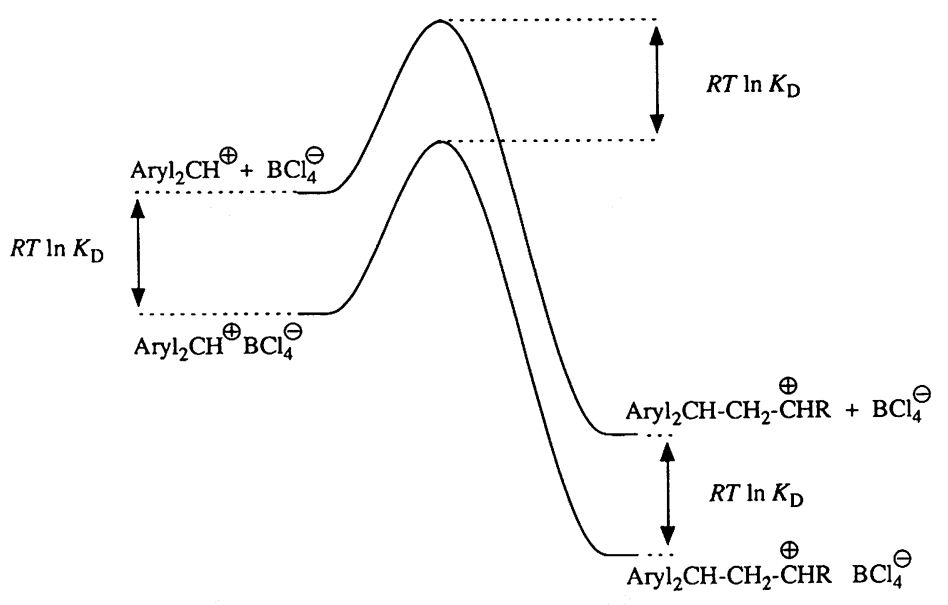

Abb. 1. Energieprofile des geschwindigkeitsbestimmenden Schritts bei Additionen gepaarter und ungepaarter Carbenium-Ionen an ein Alken.

Es ist wohlbekannt, daß die Dissoziationskonstante $K_{\mathrm{D}}$ metallorganischer Verbindungen von der Natur des Kations wie von der Struktur des Carbanions abhängig ist ${ }^{[42]}$. In Einklang damit wurden große Reaktivitätsunterschiede zwischen freien und gepaarten Carbanionen beobachtet ${ }^{[43,44]}$.

In einigen Fällen wurden Energieprofile der gesamten in Schema 15 skizzierten Reaktionsfolge einschließlich des vorausgehenden Ionisationsschritts bestimmt ${ }^{[14]}$. Dabei zeigte es sich, daß üblicherweise ein rascher Ionenkombinationsschritt dem langsamen Angriff des Carbenium-Ions auf das Alken folgt.

$\mathrm{Da}$ im geschwindigkeitsbestimmenden Schritt somit ein Carbenium-Ion aus einem anderen Carbenium-Ion hervor- 
geht, ist es nicht überraschend, daß eine Variation des Lösungsmittels nur einen geringen Einfluß auf die Reaktionsgeschwindigkeit ausübt. Für die Umsetzung von $\mathrm{An}_{2} \mathrm{CH}^{\oplus}$ mit 2-Methyl-1-penten fanden wir eine lineare Korrelation mit dem Lösungsmittel-Parameter $E_{\mathrm{T}}(30)^{[45]}$; in Nitromethan $\left(E_{\mathrm{T}}=46.3\right)$ verlief diese Addition 4.8mal rascher als in Chloroform $\left(E_{\mathrm{T}}=39.1\right)^{[14]}$.

Für die Umsetzung von $\mathrm{AnPhCD}^{\oplus}$ mit einigen Alkenen wurden sekundäre kinetische Isotopeneffekte bestimmt. Ihre Größe $\left(k_{\mathrm{D}} / k_{\mathrm{H}} \approx 1.2\right)$ deutet darauf hin, daß im Übergangszustand die Umhybridisierung des Benzhydryl-C-Atoms $\left(\mathrm{sp}^{2} \rightarrow \mathrm{sp}^{3}\right)$ weit fortgeschritten ist ${ }^{[14]}$.

\subsection{Variation der Carbenium-Ionen}

Erwartungsgemäß führt eine Verminderung des Elektronenschubs der $p$-Substituenten zu einer Erhöhung der Reaktivität der Diarylcarbenium-Ionen, die nach Tabelle 3 auf eine Absenkung der Aktivierungsenthalpie $\Delta H^{\ddagger}$ zurückzuführen ist. Dabei bleibt die Aktivierungsentropie innerhalb einer Reaktionsserie annähernd konstant. Eine Interpretation der negativen Werte von $\Delta H^{\neq}$wurde versucht ${ }^{[46]}$.

$\mathrm{Aryl}_{2} \mathrm{CHCl}+\mathrm{BCl}_{3} \underset{-70^{\circ} \mathrm{C}}{\longrightarrow} \mathrm{Aryl}_{2} \mathrm{CH}^{\oplus} \mathrm{BCl}_{4}^{\ominus}$

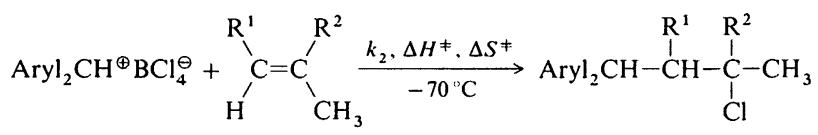

Die Korrelationen der in Tabelle 3 angeführten Reaktivitäten mit $\sum \sigma^{+}$sind nur von geringer Güte, da in den Diarylcarbenium-Ionen ungleich substituierte Arylreste in unterschiedlichem Maße aus der Ebene herausgedrängt werden, so daß die Substituenten-Effekte nicht additiv sind ${ }^{[14,47]}$. Auftragungen der Freien Aktivierungsenthalpie der Additionen $\left(\Delta G^{\ddagger}\right)$ gegen die Freie Ionisationsenthalpie der Benzhydrylchloride (Abb. 2) ergeben lineare Beziehungen mit Steigungen zwischen 0.64 und 0.94. Daraus folgt, daß im Übergangszustand der Additionen der Carbenium-Charakter des Diarylmethyl-Fragments zu mehr als 2/3 verlorengegangen ist, d.h. es liegen Produkt-ähnliche Übergangszustände vor.

Abbildung 3, in der die nicht-ionisierten Benzhydrylchloride aufeinander projiziert sind, macht deutlich, daß im Übergangszustand der Additionsreaktion die Stabilitätsrei-

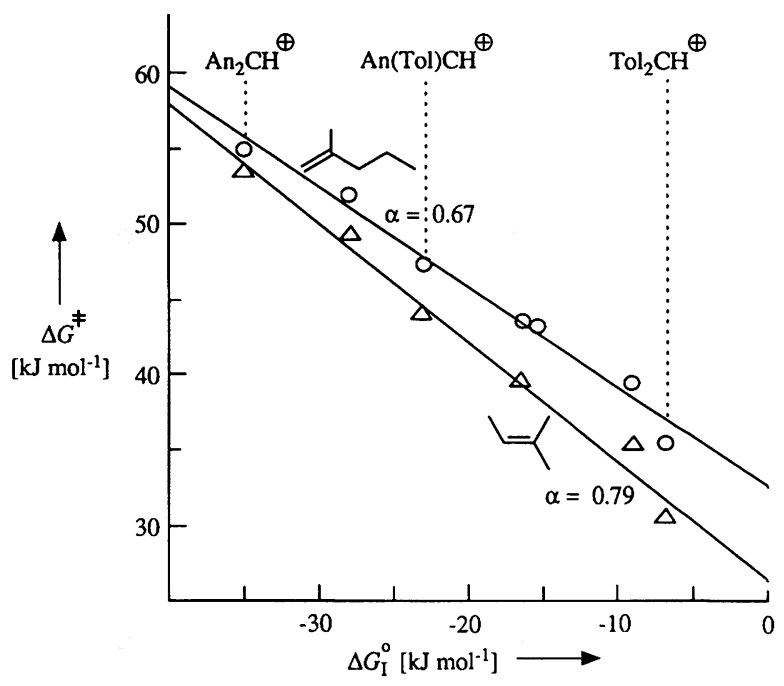

Abb. 2. Korrelation zwischen $\Delta G^{*}$ der Reaktionen von DiarylcarbeniumIonen mit Alkenen [Gl. (h)] und $\Delta G_{\mathrm{i}}^{\circ}$ der Ionisation der entsprechenden Diarylmethylchloride [Gl. (g)].

hung der Benzhydryl-Kationen zwar noch erhalten, die Aufspaltung der Energieniveaus jedoch viel geringer ist als im Grundzustand.

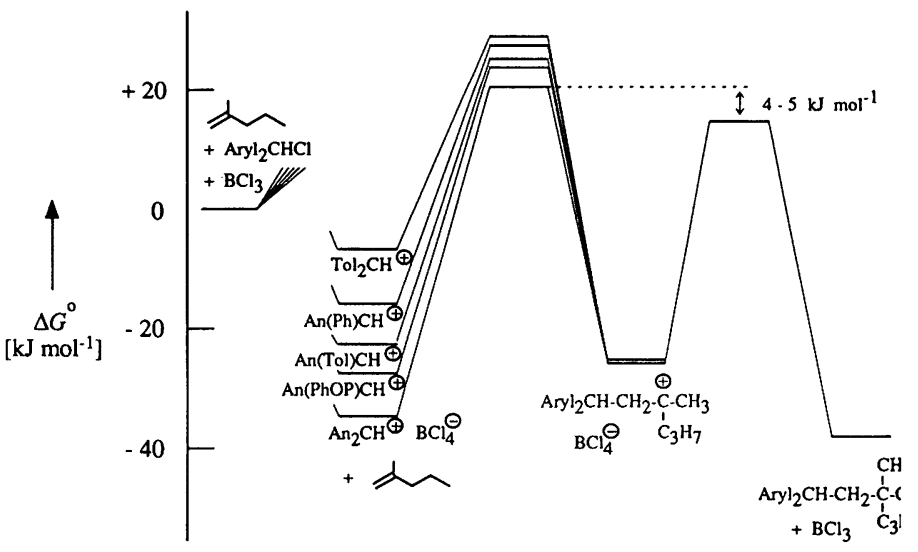

Abb. 3. Freie-Enthalpie-Profile der Bortrichlorid-induzierten Additionen von Diarylmethylchloriden an 2-Methyl-1-penten [14]

In Konkurrenzexperimenten nach Schema 14 beobachtet man eine Umkehr der vorher beschriebenen ElektrophilieReihe, wenn lediglich katalytische Mengen einer Lewis-Säure eingesetzt werden ${ }^{[2 a, 30]}$. Nunmehr ist aufgrund des höheren Ionisationsgrads das Benzhydrylchlorid reaktiver, das die besser stabilisierten Carbenium-Ionen bildet, denn

Tabelle 3. Freie Ionisationsenthalpien $\Delta G_{\mathrm{i}}^{\circ}$ von Diarylmethylchloriden [Gl. (g)] und Kinetik der Additionen von Diarylcarbenium-Ionen an 2-Methyl-1-penten und 2-Methyl-2-buten [GI. (h)] in Dichlormethan [14, 46].

\begin{tabular}{|c|c|c|c|c|c|c|c|}
\hline \multirow[b]{2}{*}{ Carbenium-Ion } & \multicolumn{4}{|c|}{ 2-Methyl-1-penten } & \multicolumn{3}{|c|}{ 2-Methyl-2-buten } \\
\hline & $\begin{array}{l}\Delta G_{\mathrm{i}}^{0}\left(-70^{\circ} \mathrm{C}\right) \\
{\left[\mathrm{kJ} \mathrm{mol}^{-1}\right]}\end{array}$ & $\begin{array}{l}k_{2}\left(-70^{\circ} \mathrm{C}\right) \\
{\left[\mathrm{L} \mathrm{mol}{ }^{-1} \mathrm{~s}^{-1}\right]}\end{array}$ & $\begin{array}{l}\Delta H^{\mp} \\
{\left[\mathrm{kJ} \mathrm{mol}^{-1}\right]}\end{array}$ & $\begin{array}{l}\Delta S^{\neq} \\
{\left[\mathrm{J} \mathrm{mol}{ }^{-1} \mathrm{~K}^{-1}\right]}\end{array}$ & $\begin{array}{l}k_{2}\left(-70^{\circ} \mathrm{C}\right) \\
{\left[\mathrm{L} \mathrm{mol}{ }^{-1} \mathrm{~s}^{-1}\right]}\end{array}$ & $\begin{array}{l}\Delta H^{\neq} \\
{\left[\mathrm{kJ} \mathrm{mol}^{-1}\right]}\end{array}$ & $\begin{array}{l}\Delta S^{*} \\
{\left[\mathrm{~J} \mathrm{~mol}^{-1} \mathrm{~K}^{-1}\right]}\end{array}$ \\
\hline $\mathrm{An}_{2} \mathrm{CH}^{\oplus}$ & -35.1 & $2.92 \times 10^{-2}$ & 29.7 & -125 & $8.38 \times 10^{-2}$ & 21.9 & -155 \\
\hline $\mathrm{An}(\mathrm{PhOP}) \mathrm{CH}^{\oplus}$ & -27.9 & $1.69 \times 10^{-1}$ & & & $7.5 \times 10^{-1}$ & & \\
\hline $\mathrm{An}(\mathrm{Tol}) \mathrm{CH}^{\oplus}$ & -22.8 & 3.38 & 22.7 & -120 & $1.83 \times 10^{1}$ & 13.6 & -151 \\
\hline $\mathrm{An}(\mathrm{Ph}) \mathrm{CH}^{\oplus}$ & -16.6 & $2.58 \times 10^{1}$ & 18.6 & -123 & $2.47 \times 10^{2}$ & 7.5 & -159 \\
\hline $\mathrm{PhOP}(\mathrm{Tol}) \mathrm{CH}^{\oplus}$ & -15.4 & $3.30 \times 10^{1}$ & & & & & \\
\hline $\mathrm{PhOP}(\mathrm{Ph}) \mathrm{CH}^{\oplus}$ & -9.2 & $2.86 \times 10^{2}$ & 15.3 & -119 & $3.92 \times 10^{3}$ & 5.3 & -147 \\
\hline $\mathrm{Tol}_{2} \mathrm{CH}^{\oplus}$ & -7.0 & $3.40 \times 10^{3}$ & 11.6 & -117 & $6.79 \times 10^{4}$ & -1.4 & -156 \\
\hline
\end{tabular}




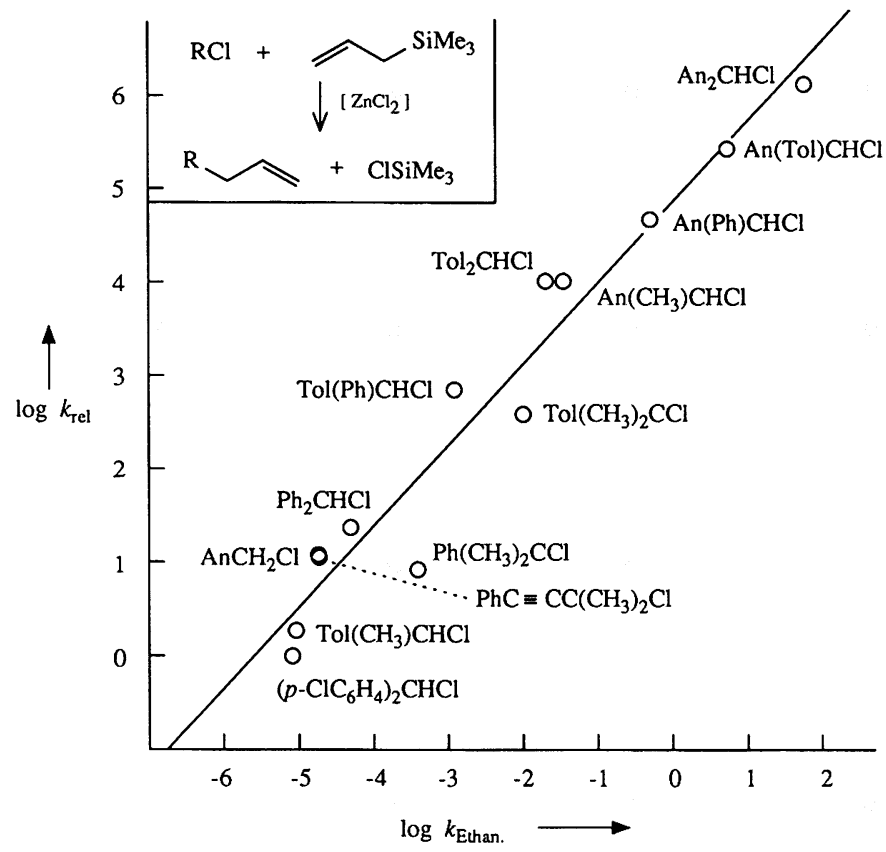

Abb. 4. Auftragung der relativen Reaktivitäten von Alkylchloriden gegenüber Allyltrimethylsilan (aus Konkurrenzexperimenten mit $\mathrm{ZnCl}_{2}$ in $\mathrm{CH}_{2} \mathrm{Cl}_{2}$ bei $-70^{\circ} \mathrm{C}$ ) [49] gegen die Logarithmen der Ethanolysegeschwindigkeitskonstanten $\log \mathrm{k}_{\text {Fthan. }}\left(100 \% \mathrm{EtOH}, 25^{\circ} \mathrm{C}\right)[50]$.

nach dem Curtin-Hammett-Prinzip ${ }^{[48]}$ wird die relative Reaktivität allein durch die relative Höhe der Übergangszustände des CC-Verknüpfungsschritts in Abbildung 3 beschrieben, sofern die Einstellung des vorgelagerten Ionisationsgleichgewichts rasch gegenüber dem Additionsschritt ist. Die Tatsache, daß durch Variation der Lewis-Säure Elektrophilie-Reihen invertiert werden können, wurde bei Synthesen genutzt ${ }^{[2 a]}$. Obwohl die kinetischen Untersuchungen bisher vorwiegend mit Diarylcarbenium-Ionen durchgeführt worden sind, sind die daraus gezogenen Schlußfolgerungen auf andere Systeme übertragbar, wie Abbildung 4 zeigt. Die relative Reaktivität von Benzhydryl-, Phenethyl-, Cumylund Propargylchloriden gegenüber Allyltrimethylsilan (das als substituiertes Ethylen betrachtet werden kann, siehe Abb. 5-7) ist in Gegenwart katalytischer Mengen Zinkchlorid den Ethanolysegeschwindigkeiten dieser Verbindungen proportional. Da die Ethanolysegeschwindigkeiten ein Maß für die ,Stabilität" der dabei auftretenden Carbenium-Ionen sind $^{[50]}$, stellt Abbildung 4 einen experimentellen Beleg dafür dar, daß in Gegenwart katalytischer Mengen Lewis-Säure die relative Elektrophilie von Alkylchloriden mit der „Stabilität" der intermediären Carbenium-Ionen zunimmt.

Die über sechs Zehnerpotenzen reichende Reaktivitätskorrelation in Abbildung 4 bedeutet, daß bei diesen Systemen sterische Effekte gegenüber den elektronischen Einflüssen vernachlässigbar sind. Die Grenze dieser Näherung zeigt sich jedoch daran, daß Triarylcarbenium-Ionen aufgrund ihres großen Raumbedarfs um ein Vielfaches langsamer reagieren als aufgrund ihrer ,Stabilität" $\left(\mathrm{p} K_{\mathrm{R} \oplus}\right.$ oder Solvolysegeschwindigkeit) zu erwarten wäre ${ }^{[29,49]}$.

\subsection{Variation der Alkene}

Aus stereochemischen Befunden (Abschnitt 2) wurde gefolgert, daß der Angriff von Carbenium-Ionen auf Alkene über wenig verbrückte Übergangszustände erfolgt, so daß unterschiedliche Effekte durch Substituenten am $\alpha$-Kohlenstoffatom (dem sich entwickelnden Carbenium-Zentrum) und am $\beta$-Kohlenstoffatom (dem Angriffspunkt des Elektrophils) zu erwarten sind (siehe 36 ).<smiles>[Y16]CC1([R])[C@@H]([R])C1([R])[R]</smiles>

\subsection{1. $\alpha$-Substituenteneffekte}

Zur Charakterisierung der Ladungsverteilung in aktivierten Komplexen wird häufig die Hammett-Reaktionskonstante $\varrho$ verwendet ${ }^{[51]}$. Aus Konkurrenzexperimenten mit in situ erzeugten Ditolylcarbenium-Ionen (Schema 17) ergaben sich Hammett-Korrelationen mäßiger Güte mit den Reaktionskonstanten $\varrho^{+}=-5.0(\mathrm{r}=0.993)$ oder $\varrho=-5.2(\mathrm{r}=$ $0.984)^{[23]}$

\begin{tabular}{|c|c|c|c|c|c|}
\hline$x$ & $\mathrm{H}$ & $p-\mathrm{F}$ & $p-\mathrm{Br}$ & $p-\mathrm{Cl}$ & $m-\mathrm{NO}_{2}$ \\
\hline$k_{\mathrm{rel}}$ & 1.00 & 1.14 & 0.26 & 0.26 & 0.00031 \\
\hline
\end{tabular}

Schema 17. Siehe [23].

Die Reaktionskonstanten liegen damit in der gleichen Größenordnung wie bei der Protonierung $\left(\mathrm{Q}^{+}=-3.6\right.$ bis $-5.4, \varrho=-4.8$ bis -7.0$)^{[52]}$ und Bromierung von Styrolen $\left(\mathrm{Q}^{+}=-4.2\right)^{[53]}$ und sind deutlich negativer als bei NOCl-Additionen $\left(\mathrm{Q}=-3.84, \mathrm{Q}^{+}=-2.08\right)^{[54]}$ oder Additionen des 2,4-Dinitrophenylsulfenylchlorids $(\varrho=-2.33$, $\left.\mathrm{Q}^{+}=-2.03\right)^{[54]}$. Die daraus für den Übergangszustand $\mathrm{ab}$ leitbare hohe positive Ladungsdichte am $\alpha$-Kohlenstoffatom ist in Einklang mit der starken Abhängigkeit der Reaktionsgeschwindigkeit von $\mathrm{R}$ (Tabelle 4) ${ }^{[6]}$

Tabelle 4. Geschwindigkeitskonstanten für die Umsetzung 2-substituierter Propene mit dem $p$-Methoxy-substituierten Diphenylmethyl-Kation $\left(\mathrm{CH}_{2} \mathrm{Cl}_{2}\right.$, $\left.-70^{\circ} \mathrm{C}\right): \mathrm{An}(\mathrm{Ph}) \mathrm{CH}^{\oplus}+\mathrm{H}_{2} \mathrm{C}=\mathrm{C}\left(\mathrm{CH}_{3}\right) \mathrm{R}$.

\begin{tabular}{lcc}
\hline $\mathrm{R}$ & $k_{2}\left[\mathrm{~L} \mathrm{~mol}^{-1} \mathrm{~s}^{-1}\right]$ & $k_{\text {rel }}$ \\
\hline $\mathrm{C} \equiv \mathrm{CH}$ & keine Reaktion & $<0.1$ \\
$\mathrm{H}$ & $9.39 \times 10^{-4}$ & 1.0 \\
$\mathrm{CH}_{3}$ & 23.3 & $2.5 \times 10^{4}$ \\
$\mathrm{CH}=\mathrm{CH}_{2}$ & 15.6 & $1.7 \times 10^{4}$ \\
$\mathrm{C}_{6} \mathrm{H}_{5}$ & $1.45 \times 10^{3}$ & $1.5 \times 10^{6}$ \\
Cyclopropyl & $5.65 \times 10^{3}$ & $6.0 \times 10^{6}$ \\
\hline
\end{tabular}

Nach Tabelle 4 nimmt die Reaktionsgeschwindigkeit um den Faktor $25000 \mathrm{zu}$, wenn das H-Atom in Position 2 von Propen durch eine Methylgruppe ersetzt wird ( $\rightarrow$ Isobuten). Der daraus ableitbare Wert $\Delta \Delta G^{\ddagger}=17 \mathrm{~kJ} \mathrm{~mol}^{-1}$ entspricht etwa der Hälfte des Unterschieds der Protonenaffinitäten von Propen und Isobuten in dem nicht-nucleophilen Solvens $\mathrm{SO}_{2} \mathrm{ClF}^{[36 \mathrm{~b}]}$. Während eine Vinylgruppe (Isopren) etwa denselben aktivierenden Effekt wie eine Methylgruppe ausübt, 
beschleunigen Phenyl und Cyclopropyl millionenfach. Überraschenderweise ist die Ethinylgruppe im Vergleich zum Wasserstoffatom sogar desaktivierend, so daß 2-Methyl-1buten-3-in unter Bedingungen inert ist, unter denen Propen glatt mit Elektrophilen reagiert.

Etwas andersartige Substituenteneinflüsse beobachtet man jedoch, wenn die Variation von $\mathrm{R}$ an einem sekundären Kohlenstoffatom durchgeführt wird (Tabelle 5) ${ }^{[6]}$. Butadien

Tabelle 5. Geschwindigkeitskonstanten für die Umsetzung monosubstituierter Ethylene mit dem $p$-Methoxy-substituierten Diphenylmethyl-Kation $\left(\mathrm{CH}_{2} \mathrm{Cl}_{2}\right.$, $\left.-70^{\circ} \mathrm{C}\right): \mathrm{An}(\mathrm{Ph}) \mathrm{CH}^{\oplus}+\mathrm{H}_{2} \mathrm{C}=\mathrm{CHR}$.

\begin{tabular}{lll}
\hline $\mathrm{R}$ & $k_{2}\left[\mathrm{~L} \mathrm{~mol}^{-1} \mathrm{~s}^{-1}\right]$ & $k_{\text {rel }}$ \\
\hline $\mathrm{CH}_{3}$ & $9.39 \times 10^{-4}$ & 1.0 \\
$\mathrm{CH}=\mathrm{CH}_{2}$ & $1.93 \times 10^{-2}$ & 21 \\
$\mathrm{C}_{6} \mathrm{H}_{5}$ & 10.9 & $1.2 \times 10^{4}$ \\
$\mathrm{CH}=\mathrm{CH}\left(\mathrm{CH}_{3}\right)$ & 46.2 & $4.9 \times 10^{4}$ \\
\hline
\end{tabular}

ist nämlich 21 mal reaktiver als Propen, woraus hervorgeht, daß nun eine Vinylgruppe deutlich stärker aktiviert als eine Methylgruppe. Die beim trans-Piperylen noch hinzukommende Methylgruppe verursacht einen Geschwindigkeitsanstieg um 2400 gegenüber Butadien. Deutlich stärker als in Tabelle 4 kommt nun der aktivierende Effekt eines Phenylrests zum Tragen: Während $\alpha$-Methylstyrol nur $62 \mathrm{mal}$ reaktiver ist als Isobuten, reagiert Styrol 12000 mal rascher als Propen. Damit ist Styrol gegenüber Carbenium-Ionen ähnlich nucloeophil wie Isobuten; dies ist in Einklang mit der Faustregel, daß Carbenium-Ionen durch zwei Methylgruppen etwa gleich gut stabilisiert werden wie durch eine Phenylgruppe $^{[55]}$. Gegenüber anderen Elektrophilen ist Isobuten jedoch bis zu $1000 \mathrm{mal}$ reaktiver als Styrol ${ }^{[56]}$.

Eine Verzweigung des $\alpha$-Substituenten in allylischer Position führt zu einer mäßigen Reaktivitätsminderung: Die tertButyl-substituierte Verbindung in Schema 18 reagiert nur 19 mal langsamer als Isobuten.

$\mathrm{An}(\mathrm{Ph}) \mathrm{CH}^{\oplus}+\mathrm{H}_{2} \mathrm{C}=\mathrm{C}\left(\mathrm{CH}_{3}\right) \mathrm{R}\left(\mathrm{CH}_{2} \mathrm{Cl}_{2},-70^{\circ} \mathrm{C}\right)$

\begin{tabular}{lllll}
\hline $\mathrm{R}$ & $\mathrm{Me}$ & $\mathrm{Et}$ & $i \mathrm{Pr}$ & $t \mathrm{Bu}$ \\
\hline$k_{2}\left[\mathrm{~L} \mathrm{~mol}^{-1} \mathrm{~s}^{-1}\right]$ & 23.3 & 18.4 & 6.08 & 1.21 \\
\hline
\end{tabular}

Schema 18. Effekt von Methylgruppen in Allyl-Stellung [6].

Dagegen beobachtet man sogar eine geringfügige Reaktivitätssteigerung, wenn die Verzweigung in der homoallylischen Position erfolgt. Der aktivierende hyperkonjugative Effekt vermag nunmehr den sterischen Effekt zu überspielen (Schema 19).

$\mathrm{An}(\mathrm{Ph}) \mathrm{CH}^{\oplus}+\mathrm{H}_{2} \mathrm{C}=\mathrm{C}\left(\mathrm{CH}_{3}\right) \mathrm{CH}_{2}-\mathrm{R}\left(\mathrm{CH}_{2} \mathrm{Cl}_{2},-70^{\circ} \mathrm{C}\right)$

\begin{tabular}{llll}
\hline $\mathrm{R}$ & $\mathrm{Me}$ & $\mathrm{Et}$ & $t \mathrm{Bu}$ \\
\hline$k_{2}\left[\mathrm{~L} \mathrm{~mol}^{-1} \mathrm{~s}^{-1}\right]$ & 18.4 & 25.8 & 28.6 \\
\hline
\end{tabular}

Schema 19. Effekt von Methylgruppen in Homoallyl-Stellung [6].

\subsection{2. $\beta$-Substituenteneffekte}

Während die $\alpha$-Substituenteneffekte im wesentlichen enthalpischer Natur sind, resultieren die $\beta$-Effekte aus einem komplexen Zusammenspiel enthalpischer und entropischer Einflüsse. Weiterhin ist zu beachten, daß die Größe der $\alpha$-Effekte von der Natur des Referenzkations unabhängig ist, während sich die $\beta$-Effekte verändern, wenn das BenzhydrylKation variiert wird (vgl. Abschnitt 5.4). Generell beobachtet man jedoch, daß die Einführung einer Methylgruppe in $\beta$-Position zu einer Vergrößerung des negativen Betrags von $\Delta S \neq$ führt. Gleichzeitig wird auch die Aktivierungsenthalpie $(\Delta H \neq)$ gesenkt (Ausnahme: cis-1-Phenylpropen), so daß eine Methylgruppe in $\beta$-Stellung den elektrophilen Angriff erleichtern oder erschweren kann (Tabelle 6). Die Diskussion

Tabelle 6. Der Einfluß von $\beta$-Methylgruppen an Alkenen auf deren Reaktivität gegenüber dem Methoxy-substituierten Benzhydryl-Kation $\mathrm{An}(\mathrm{Ph}) \mathrm{CH}^{\oplus}\left(\mathrm{CH}_{2} \mathrm{Cl}_{2}\right.$. $\left.-70^{\circ} \mathrm{C}\right)$.

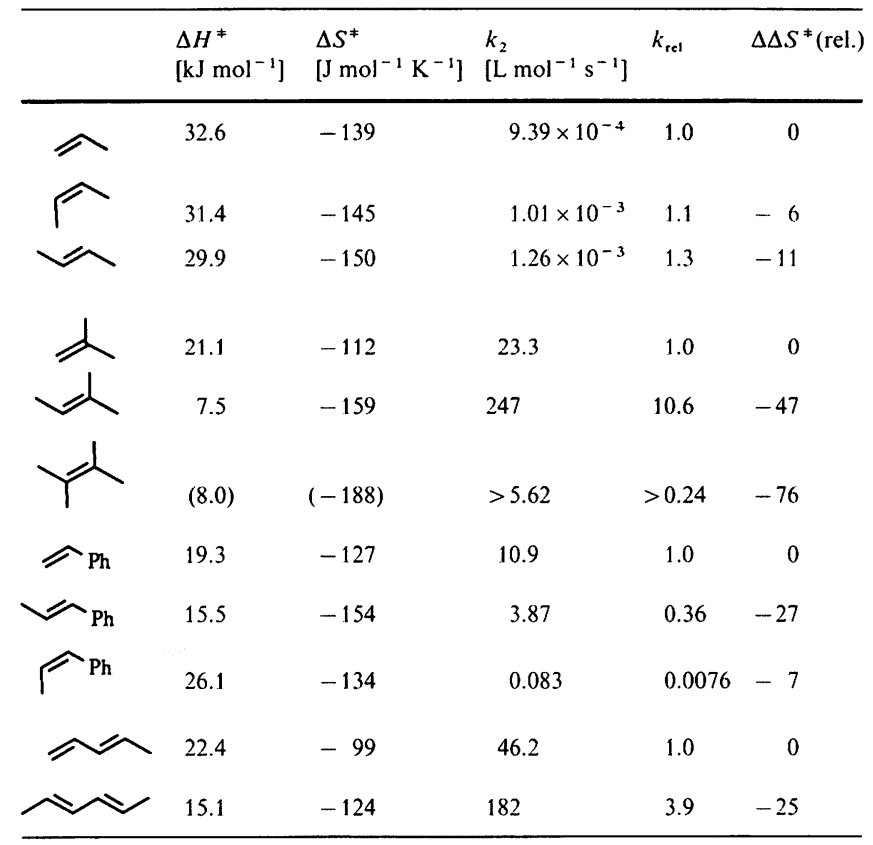

des Einflusses der zweiten $\beta$-Methylgruppe ist problematisch, weil bei der Umsetzung von Diarylcarbenium-Ionen mit Tetramethylethylen der CC-Verknüpfungsschritt nicht geschwindigkeitsbestimmend ist ${ }^{[21]}$.

\begin{tabular}{lcc}
$\mathrm{An}(\mathrm{Ph}) \mathrm{CH}^{\oplus}+\mathrm{RHC}=\mathrm{C}\left(\mathrm{CH}_{3}\right)_{2}\left(-70{ }^{\circ} \mathrm{C}, \mathrm{CH}_{2} \mathrm{Cl}_{2}\right)$ & \\
\hline $\mathrm{R}$ & $\mathrm{CH}_{3}$ & $t \mathrm{Bu}$ \\
\hline$k_{2}\left[\mathrm{~L} \mathrm{~mol}^{-1} \mathrm{~s}^{-1}\right]$ & 247 & $<0.247$ \\
\hline
\end{tabular}

Schema 20. Siehe [6],

Eine massive sterische Behinderung wird beobachtet, wenn eine tert-Butylgruppe in $\beta$-Position eingeführt wird. So reagiert 2 mindestens 1000 mal langsamer als 2-Methyl-2-buten (Schema 20). Da 2 unter Säurekatalyse in das wesentlich reaktivere 37 umlagert, ist dieser Quotient sogar als untere Grenze anzusehen. 
<smiles>C=C(C)CC(C)(C)C</smiles>

\subsubsection{Cyclische Alkene und Diene}

Cycloalkene und Methylencycloalkane unterscheiden sich in ihrer Nucleophilie zum Teil erheblich von ihren acyclischen Analoga ${ }^{[6,36 \mathrm{~b}]}$. Da die Gründe hierfür noch weitgehend unverstanden sind, sei auf eine Diskussion an dieser

\begin{tabular}{lcccc}
$\mathrm{An}(\mathrm{Ph}) \mathrm{CH}^{\oplus}+$ & \multicolumn{5}{c}{$\left.\mathrm{CH}_{2}\right)_{\mathrm{n}}\left(-70^{\circ} \mathrm{C}, \mathrm{CH}_{2} \mathrm{Cl}_{2}\right)$} & & \\
\hline $\mathrm{n}$ & 1 & 2 & 3 & 4 \\
\hline$k_{2}\left[\mathrm{~L} \mathrm{~mol}^{-1} \mathrm{~s}^{-1}\right]$ & 1740 & 27.5 & 3.04 & 0.326 \\
\hline
\end{tabular}

Schema 21

Stelle verzichtet. In der Reihe der Cycloalkadiene bewirkt zunehmende Störung der Planarität des $\pi$-Elektronensystems eine Verminderung der Nucleophilie (Schema 21).

\subsubsection{Vergleich mit anderen Nucleophilie-Reihen}

Kinetische wie stereochemische Befunde deuten auf einen nur wenig verbrückten Übergangszustand beim Angriff der Carbenium-Ionen auf Alkene, so daß es nicht überrascht, daß die gegenüber Carbenium-Ionen beobachteten Reaktivitätsabstufungen nicht mit den Reaktivitätsreihen gegenüber verbrückenden Elektrophilen korreliert sind. Aus Tabelle 7 geht beispielsweise die ähnliche Größe von $\alpha$ - und $\beta$-Methyleffekten bei Bromierung hervor ${ }^{[57]}:(E)$-2-Buten und Isobu-

Tabelle 7. Relative Reaktivitäten von $\pi$-Nucleophilen gegenüber einigen Elektrophilen.

\begin{tabular}{|c|c|c|c|c|c|}
\hline & & & & & \\
\hline $\mathrm{An}(\mathrm{Ph}) \mathrm{CH}^{\oplus}\left(-70^{\circ} \mathrm{C}\right)[\mathrm{a}]$ & 1.00 & 1.34 & $2.48 \times 10^{4}$ & $1.66 \times 10^{4}$ & $1.16 \times 10^{4}$ \\
\hline $\mathrm{Br}_{2}\left(\mathrm{CH}_{3} \mathrm{OH}, 25^{\circ} \mathrm{C}\right)[\mathrm{b}]$ & 1.00 & 32.3 & 92.3 & & 3.80 \\
\hline $\mathrm{H}_{3} \mathrm{O}^{\oplus}\left(\mathrm{H}_{2} \mathrm{SO}_{4}, 25^{\circ} \mathrm{C}\right)[\mathrm{c}]$ & 1.00 & 14.8 & $1.56 \times 10^{5}$ & $1.34 \times 10^{4}$ & $1.01 \times 10^{2}$ \\
\hline
\end{tabular}

[a] Lit. [6]. [b] Lit. [57]. [c] Lit. [58]

ten werden mit vergleichbarer Geschwindigkeit bromiert, während Styrol deutlich reaktionsträger ist. Bei Protonierungen beobachtet man wie bei Additionen von CarbeniumIonen stark unterschiedliche $\alpha$ - und $\beta$-Methyleffekte (Isobuten $/(E)$-2-Buten $=10^{4}$; Tabelle 7$)$, doch bewirkt $\pi$-Konjugation eine viel schwächere Aktivierung als bei den Additionen von Carbenium-Ionen. So ist die Protonierung von Isopren um eine Zehnerpotenz, die von Styrol um drei Zehnerpotenzen langsamer als die von Isobuten, während diese drei Verbindungen mit Carbenium-Ionen fast gleich rasch reagieren.

Der Vergleich der Reaktivitäten von zwanzig Alkenen und Dienen gegenüber $\mathrm{H}_{3} \mathrm{O}^{\oplus}$ und $\mathrm{An}(\mathrm{Ph}) \mathrm{CH}^{\oplus}$ zeigte, daß die beiden Datensätze allenfalls mäßig miteinander korreliert sind $(r=0.71)^{[6]}$, so daß wir derzeit keine Möglichkeit sehen, Reaktivitäten gegenüber Carbenium-Ionen aus anderen Reaktivitätsreihen vorauszusagen. In Einklang mit dem postulierten späten Übergangszustand der Additionen von Carbenium-Ionen fand man jedoch eine brauchbare Korrelation $(r=0.91)$ zwischen den Reaktionsgeschwindigkeiten der Alkene und den Solvolysegeschwindigkeiten der Additionsprodukte ${ }^{[6]}$.

\subsection{Lineare Freie-Enthalpie-Beziehungen}

Korrelationen höherer Güte als in Abbildung 2 ergeben sich, wenn die Reaktivitäten der Alkene gegenüber Diarylcarbenium-Ionen untereinander verglichen werden, d.h. wenn als Bezugsskala die Reaktivität dieser Carbenium-Ionen gegen ein Referenznucleophil gewählt wird. Abbildung 5 zeigt, daß die für terminale Alkene resultierenden Reaktivitäts-Reaktivitäts-Korrelationen annähernd parallele Geraden ergeben, d.h. die relative Reaktivität dieser Verbindungen ist unabhängig vom Elektrophil ${ }^{[46]}$.

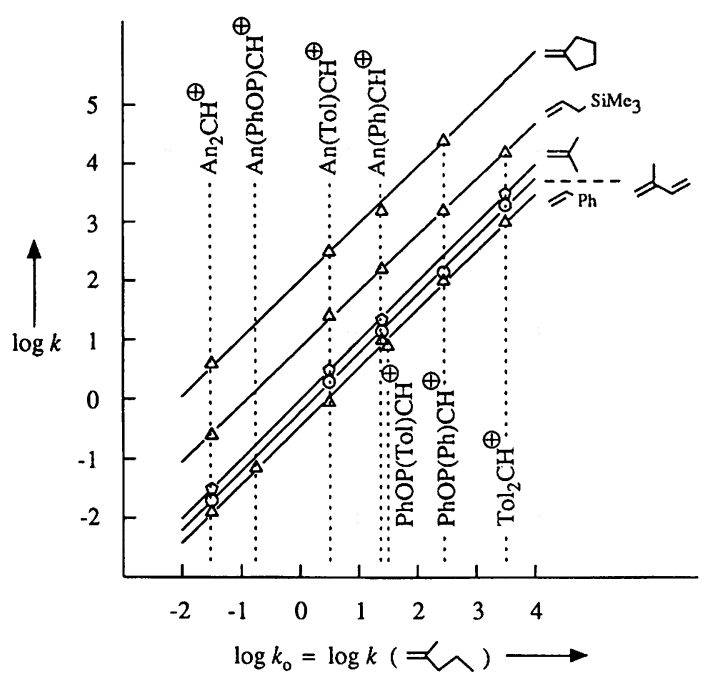

Abb. 5. Reaktivitäten von terminalen Alkenen gegenüber DiarylcarbeniumIonen $\left(-70^{\circ} \mathrm{C}, \mathrm{CH}_{2} \mathrm{Cl}_{2}\right.$, Referenzreaktion: $\mathrm{Aryl}_{2} \mathrm{CH}^{\oplus}+2-\mathrm{Methyl}-1$-penten) [46].

Eine alternative Darstellung dieser Beziehungen (Abb. 6) zeigt, daß die Selektivität der Carbenium-Ionen gegenüber einem Paar von Nucleophilen konstant bleibt, selbst wenn die Reaktivität um acht Zehnerpotenzen variiert wird. Man findet somit ,constant selectivity correlations“ im Sinne von Ritchie $^{[59]}$, obwohl die gängige Erklärung dieser Beziehungen durch Solvatationseffekte ${ }^{[60]}$ hier versagt.

Es ist daher zu folgern, daß strukturelle Variationen in größerer Entfernung vom Reaktionszentrum keinen Einfluß auf die Struktur des Übergangszustands haben. Diese Deutung bietet sich vor allem deswegen an, weil bei Einbeziehung von Olefinen mit nicht-terminaler Doppelbindung zwar ebenfalls lineare Reaktivitäts-Reaktivitäts-Beziehungen resultieren (Abb. 7), die Geraden nun aber nicht mehr parallel verlaufen, so daß eine Veränderung der Struktur des Übergangszustands durch die Substituentenvariation am Reaktionszentrum angezeigt wird. Anders als in Abbildung 


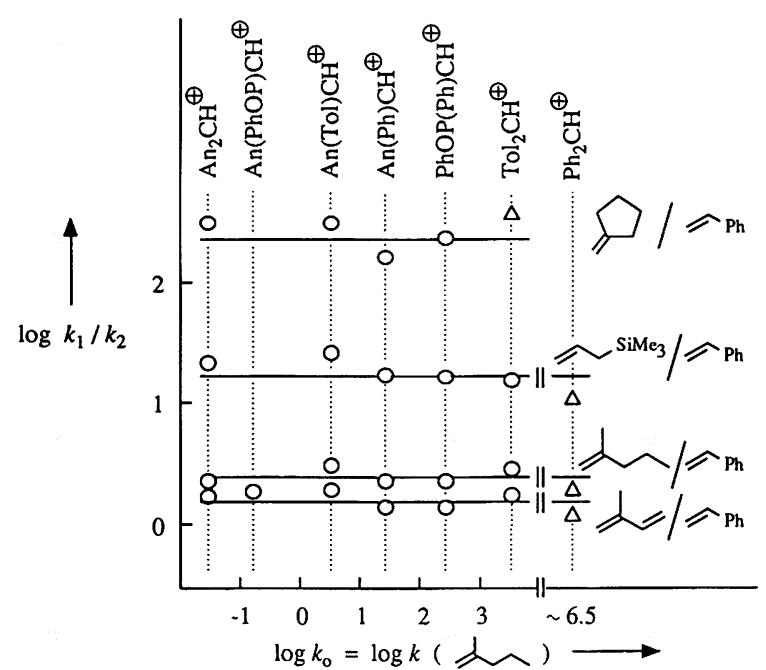

Abb. 6. Konstante Selektivität von Diarylcarbenium-Ionen gegenüber Paaren von terminalen Alkenen $\left(-70^{\circ} \mathrm{C}, \mathrm{CH}_{2} \mathrm{Cl}_{2}\right)$. Die als Dreiecke angegebenen Selektivitäten stammen aus Konkurrenz-Experimenten [23,36 b], alle anderen aus direkt gemessenen Geschwindigkeitskonstanten.

5 treten nun Überkreuzungen auf, und die gegenüber einem bestimmten Carbenium-Ion ermittelte Nucleophilie-Reihe ist nicht auf andere Elektrophile übertragbar.

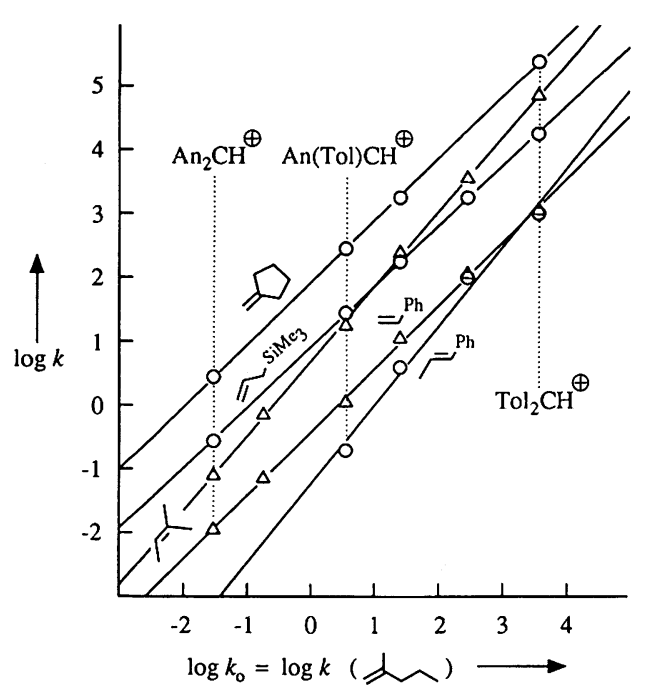

Abb. 7. Reaktivitäten von Olefinen gegenüber Diarylcarbenium-Ionen ( $-70^{\circ} \mathrm{C}, \mathrm{CH}_{2} \mathrm{Cl}_{2}$, Referenzreaktion: $\mathrm{Aryl}_{2} \mathrm{CH}^{\oplus}+2$-Methyl-1-penten) [46].

Da nach Abbildung 2 eine lineare Korrelation zwischen Reaktivität der Diarylcarbenium-Ionen und deren Grundzustandsenergie $\left(\Delta G_{\mathrm{i}}^{0}\right)$ besteht, welche nach Gleichung $(\mathrm{d}) \mathrm{mit}$ den $\mathrm{p} K_{\mathrm{R} \theta}$-Werten verknüpft ist, läßt sich für die Reaktivität von Diarylcarbenium-Ionen mit Alkenen die Gleichung (i) ableiten. Entsprechend ergibt sich aus der Korrelation von $\Delta G_{\mathrm{i}}^{0}$ mit den Ethanolysekonstanten der Benzhydryichloride die Gleichung (j) ${ }^{[46]}$.

$\log k\left(\mathrm{CH}_{2} \mathrm{Cl}_{2},-70^{\circ} \mathrm{C}\right)=-7.42 \cdot s-1.07 \cdot s \cdot \mathrm{p} K_{\mathrm{R} \oplus}+c$

$\log k\left(\mathrm{CH}_{2} \mathrm{Cl}_{2},-70^{\circ} \mathrm{C}\right)=0.90 \cdot s-1.26 \cdot s \cdot \log k_{\text {Ethan }}+c$

Einige Werte für $c$, einem Maß für die Nucleophilie der Alkene, und für $s$, einem Maß für die Empfindlichkeit, mit der die Geschwindigkeitskonstante auf Variation des Carbenium-Ions reagiert, finden sich in Tabelle 8. $\mathrm{p} K_{\mathrm{R}^{-}}$
Werte sind in Lit. ${ }^{[61]}$ und die Ethanolysegeschwindigkeiten der Benzhydrylchloride bei $25^{\circ} \mathrm{C}\left(\log k_{\text {Ethan }}\right)$ in Lit. ${ }^{[31]} \mathrm{zu}$ sammengestellt. Da die Steigungen der Reaktivitäts-Reaktivitäts-Korrelationen in Abbildung 7 hauptsächlich von der Substitution am elektrophil angegriffenen Kohlenstoffatom abhängig sind, können die Gleichungen (i) und (j) auch zur Berechnung der Reaktivitäten solcher Alkene genutzt werden, die in Tabelle 8 nicht enthalten sind; eine detaillierte

Tabelle 8. Alken-Parameter zur Berechnung der Reaktivitäten gegenüber Diarylcarbenium-Ionen nach Gleichung (i) und (j) [46].

\begin{tabular}{|c|c|c|}
\hline Alken & $s$ & c \\
\hline & 1.000 & 0.000 \\
\hline & 1.000 & 0.014 \\
\hline & 0.948 & 2.00 \\
\hline & 0.978 & -0.418 \\
\hline & 0.977 & -0.174 \\
\hline & 0.950 & 0.918 \\
\hline & 1.164 & 0.722 \\
\hline & 1.106 & -0.633 \\
\hline & 1.248 & -1.26 \\
\hline & 1.394 & -1.25 \\
\hline
\end{tabular}

Vorgehensweise hierfür ist beschrieben ${ }^{[46]}$. Aufgrund der in Abbildung 4 gezeigten Beziehung ist anzunehmen, daß die Korrelationen (i) und (j) auch Näherungswerte für andere Klassen von Carbenium-Ionen liefern, sofern Systeme mit großem Raumanspruch, z. B. Triphenylmethyl-Systeme ausgespart bleiben.

\subsection{Spielen SET-Prozesse eine Rolle?}

Eine Alternative zu den bisher diskutierten Mechanismen ist der in Gleichung (k) formulierte Einelektronenübergang (single electron transfer, SET) unter Bildung eines Diarylmethyl-Radikals und eines Alken-Radikalkations, dem eine Kombination dieser beiden Radikale folgt ${ }^{[62]}$.

$\mathrm{Aryl}_{2} \mathrm{CH}^{\oplus}+\mathrm{H}_{2} \mathrm{C}=\mathrm{CH}-\mathrm{R} \stackrel{\Delta E \approx 2 \mathrm{eV}}{\longrightarrow} \mathrm{Aryl}_{2} \dot{\mathrm{C}} \mathrm{H}+\left[\mathrm{H}_{2} \mathrm{C}-\mathrm{CH}-\mathrm{R}\right]^{\oplus}(\mathrm{k})$

Da dieser Schritt thermodynamisch ungünstig ist (Ionisationspotential von $\mathrm{Ph}_{2} \mathrm{CH}$. und Isobuten: 7.32 bzw. $9.23 \mathrm{eV}$ ), schlossen Dorfman et al. einen derartigen Elektronenübergang aus ${ }^{[39]}$. Obwohl sich die angegebenen Ionisationspotentiale auf die Gasphase beziehen, ist es unwahrscheinlich, daß Solvatationseffekte die Energetik stark beeinflussen, da die Polarität durch den Einelektronenübergang kaum verändert wird.

Nähme man dennoch an, daß beim Angriff von Carbenium-Ionen auf Alkene der in Gleichung $(k)$ formulierte Einelektronenübergang geschwindigkeitsbestimmend ist, sollten die Reaktionsgeschwindigkeiten mit den Ionisationspotentialen der Alkene verknüpft sein. Aus Tabelle 6 geht her- 
vor, daß Isobuten trotz seines etwas höheren Ionisationspotentials $(9.23 \mathrm{eV}) 20000 \mathrm{mal}$ reaktiver ist als die 2-Butene $(9.13 \mathrm{eV})^{[63]}$. Dieser Befund spricht wiederum gegen einen geschwindigkeitsbestimmenden SET-Schritt, doch lassen sich bei diesem Vergleich sterische Effekte als Ursache für den Reaktivitätsunterschied nicht mit Sicherheit ausschlieBen.

In Abbildung 8 sind die Reaktivitäten terminaler Alkene gegen ihre Ionisationspotentiale aufgetragen. Man erkennt,

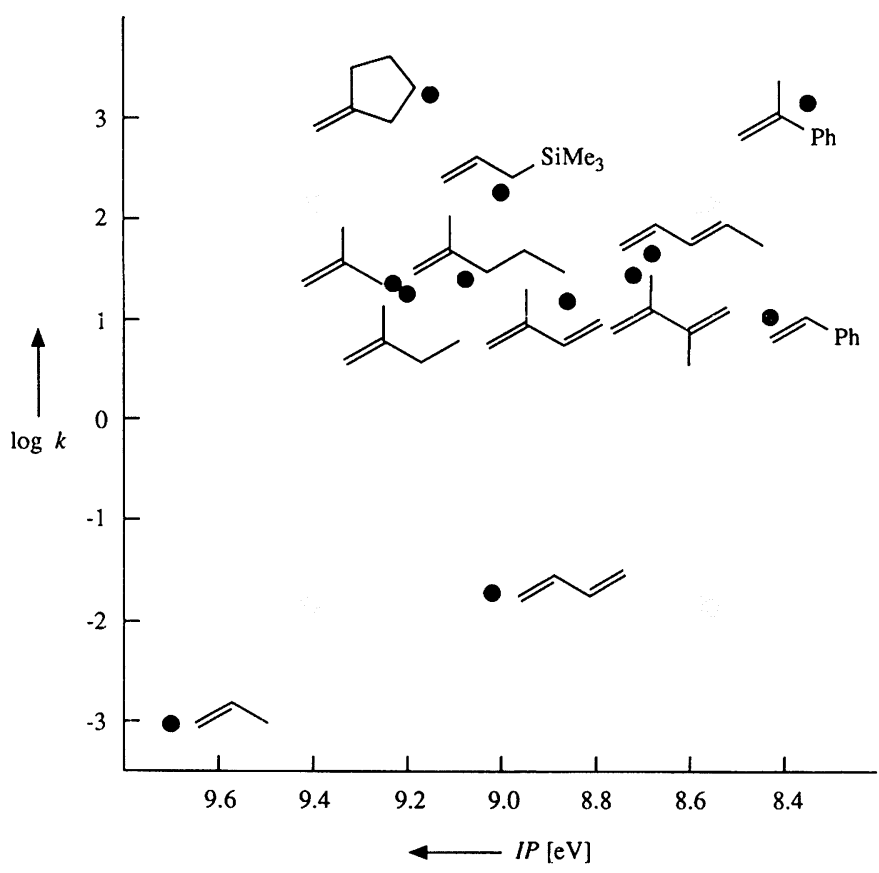

Abb. 8. Auftragung der Reaktivitäten terminaler Alkene gegenüber $\mathrm{An}(\mathrm{Ph}) \mathrm{CH}^{\oplus}$ gegen ihre Ionisationspotentiale $I P[64]$.

daß sich einerseits Butadien und Methylencyclopentan trotz ähnlicher Ionisationspotentiale in ihren Reaktivitäten um fünf Zehnerpotenzen unterscheiden. Andererseits haben Isobuten und Styrol trotz eines um $0.8 \mathrm{eV}$ verschiedenen Ionisationspotentials annähernd gleiche Reaktivität. Da aus Abbildung 5 auf strukturell ähnliche Übergangszustände beim Angriff von Diarylcarbenium-Ionen auf terminale Alkene geschlossen wurde, interpretieren wir die fehlende Korrelation zwischen Reaktivitäten und Ionisationspotentialen in Abbildung 8 als Beweis gegen einen geschwindigkeitsbestimmenden SET-Prozeß.

\section{Ausblick}

Wie eingangs geschildert, wurden für die hier beschriebenen Untersuchungen bevorzugt Diarylcarbenium-Ionen 1 als mechanistische Sonden gewählt, weil sich deren Reaktivität durch Variation der para-Substituenten modifizieren läßt, ohne die sterischen Gegebenheiten am Reaktionszentrum zu verändern. Die damit erarbeiteten Gesetzmäßigkeiten sind jedoch auf andere Elektrophile übertragbar: Kinetische Untersuchungen mit Cyclopentenylium-Ionen haben beispielsweise ergeben, daß sich die in Tabelle 4 und 5 wiedergegebenen relativen Reaktivitäten der Alkene nur gering- fügig ändern, wenn als Referenz-Elektrophile Allyl-Kationen anstelle von Benzhydryl-Kationen eingesetzt werden ${ }^{[65]}$. Es ist somit zu erwarten, daß die in Abschnitt 5.3 angegebenen Nucleophilie-Reihen der Alkene näherungsweise auf Alkylierungen von Alkenen mit beliebigen Carbenium-Ionen anwendbar sind.

Nach Abbildung 4 besteht eine Korrelation zwischen relativer Elektrophilie und Ethanolysegeschwindigkeit $\left(\mathrm{S}_{\mathrm{N}} 1-\mathrm{Re}-\right.$ aktivität) nicht nur bei Diarylmethylchloriden, sondern auch für andere $S_{N} 1$-aktive Verbindungen, einschließlich des primären $p$-Methoxybenzylchlorids und der tertiären Cumylchloride. Damit bietet sich dem Synthetiker die Möglichkeit, auf der Basis von Abbildung 4 oder Gleichung (j) relative und absolute Reaktionsgeschwindigkeiten bei Alkylierungen vorauszusagen, sofern die Solvolysegeschwindigkeiten der Carbenium-Ionen-Vorstufen bekannt sind.

Diarylcarbenium-Ionen haben sich somit als geeignete Sonden erwiesen, um Einblick in den Mechanismus des Angriffs von Carbenium-Ionen auf Alkene sowie in einige prinzipielle Zusammenhänge zwischen Reaktivität und Selektivität zu gewinnen. Da sich diese Ionen auch durch LaserpulsPhotolyse in Gegenwart von reaktiveren Olefinen (Enolether etc.) erzeugen lassen ${ }^{[66]}$, ist es möglich, die hier beschriebenen Reaktivitätsreihen bis in den Bereich diffusionskontrollierter Reaktionen weiterzuverfolgen. Kinetische Untersuchungen der Reaktionen von Diarylcarbenium-Ionen mit Allylelementverbindungen der 4. Hauptgruppe ${ }^{[67,68]}$ und mit Arenen ${ }^{[68]}$ haben gezeigt, daß diese Nucleophile den gleichen linearen Freie-Enthalpie-Beziehungen gehorchen wie Alkene. Damit eröffnet sich die Möglichkeit, eine große Vielfalt polarer CC-Verknüpfungen durch ein einheitliches mechanistisches Schema zu beschreiben. Einige der daraus für die Syntheseplanung resultierenden Konsequenzen wurden in einer kurzen Zusammenfassung dargestellt ${ }^{[2 a]}$.

Der überwiegende Teil der hier beschriebenen Arbeiten wurde von den Herren Drs. R. Pock, R. Schneider und C. Schade mit tatkräftiger Unterstützung durch $M$. Deters (geborene Rubow) und U. Grabis durchgeführt. Ihrem engagierten und zielbewußten Schaffen ist es zu verdanken, daß innerhalb weniger Jahre eine komplexe Reaktionsfolge weitgehend aufgeklärt werden konnte. Diese Arbeiten wären nicht möglich gewesen ohne die großzügigen Erstausstattungsmittel der Universität Lübeck und die stete, unbürokratische Förderung durch die Deutsche Forschungsgemeinschaft. Mein Dank gilt weiterhin dem Fonds der Chemischen Industrie sowie der Volkswagen-Stiftung für die Gewährung eines Kekulé-Stipendiums an C. Schade.

Eingegangen am 17. April 1990

[A 797]

[1] a) D. Schinzer (Hrsg.): Selectivities in Lewis Acid Promoted Reactions (NATO ASI Series C, Bd. 289), Kluwer, Dodrecht 1989; Ältere Übersichtsartikel: b) P. A. Bartlett in J. D. Morrison (Hrsg.): Asymmetric Synthesis, Vol. 3, Part B, Academic Press, New York 1984, Kap. 5 und 6; c) G. A. Olah (Hrsg.): Friedel-Crafts and Related Reactions, Wiley-Interscience, New York 1963-1965; d) G. A. Olah: Friedel-Crafts Chemistry, Wiley-Interscience, New York 1973; e) R. M. Roberts, A. A. Khalaf: Friedel-Crafts Alkylation Chemistry, M. Dekker, New York 1984.

[2] a) H. Mayr in [1 a], S. 21; b) H. Mayr, Angew. Chem. 93 (1981) 202; Angew Chem. Int. Ed. Engl. 20 (1981) 184; c) H. Mayr, W. Striepe, J. Org. Chem. 48 (1983) 1159 ; d) L. Schmerling in [1 c], Vol. II, Kap. 26; e) G. A. Olah, S. J. Kuhn, D. G. Barnes, J. Org. Chem. 29 (1964) 2685; f) H. Stetter. P. Goebel, Chem. Ber. 95 (1962) 1039; g) A. A. Petrov, K. V. Leets, J. Gen. Chem. USSR 26 (1956) 1263; h) M. L. Genusov, A. A. Petrov, ibid. 33 (1963) 2787. 
[3] a) P. B. D. De la Mare, R. Bolton: Electrophilic Additions to Unsaturated Systems, 2. Aufl., Elsevier, Amsterdam 1982, Kap. 3; b) G. H. Schmid, D. G. Garratt in S. Patai (Hrsg.): The Chemistry of Double-Bonded Functional Groups Suppl. A, Part 2, Wiley, London 1977, Kap. 9, S. 732; c) V. J. Nowlan, T. T. Tidwell, Acc. Chem. Res. 10 (1977) 252.

[4] a) H. Mayr, H. Klein, Chem. Ber. 115 (1982) 3528; b) H. Mayr, H. Klein, G. Kolberg, ibid. 117 (1984) 2555

[5] Siehe [3 b], S. 860.

[6] H. Mayr, R. Schneider, B. Irrgang, C. Schade, J. Am. Chem. Soc. 112 (1990) 4454.

[7] F. Marcuzzi, G. Melloni, J. Chem. Soc. Perkin Trans. 2 1976, 1517.

[8] F. Marcuzzi, G. Melloni, J. Am. Chem. Soc. 98 (1976) 3295.

[9] H. Groß, E. Höft, Angew. Chem. 79 (1967) 358; Angew. Chem. Int. Ed. Engl. 6 (1967) 335.

[10] M. J. S. Dewar, C. H. Reynolds, J. Am. Chem. Soc. 106 (1984) 1744.

[11] W. S. Johnson, Angew. Chem. 88 (1976) 33; Angew. Chem. Int. Ed. Engl. 15 (1976) 9; Bioorg. Chem. 5 (1976) 51; vgl. [1 b]

[12] R. Pock, H. Mayr, M. Rubow, E. Wilhelm, J. Am. Chem. Soc. 108 (1986) 7767.

[13] K. Matyjaszewski in G. Allen, J. C. Bevington (Hrsg.): Comprehensive Polymer Science, Vol. 4, Pergamon Press, Oxford 1989, Kap. 41.

[14] H. Mayr, R. Schneider, C. Schade, J. Bartl, R. Bederke, J. Am. Chem. Soc. $112(1990) 4446$

[15] a) J. H. Rolston, K. Yates, J. Am. Chem. Soc. 91 (1969) 1469, 1477; b) M. F. Ruasse, Acc. Chem. Res. 23 (1990) 87.

[16] E. von der Brüggen, Dissertation, Medizinische Universität zu Lübeck 1988.

[17] a) P. Vogel: Carbocation Chemistry, Elsevier, Amsterdam 1985, Kap. 8; b) M. Saunders, J. Chandrasekhar, P. von R. Schleyer in P. de Mayo (Hrsg.): Rearrangements in Ground and Excited States, Vol. 1, Academic Press, New York 1980, Kap. 1; c) W. Kirmse, Top. Curr. Chem. 80 (1979) 125; d) V. G. Shubin, ibid. 116/117 (1984) 267.

[18] a) M. L. Genusov, A. A. Petrov, Zh. Org. Khim 1 (1965) 2105; Chem. Abstr. 64 (1966) 11098 a; b) H. Klein, Dissertation, Universität ErlangenNürnberg 1982; c) R. Pock, Diplomarbeit, Universität Erlangen-Nürnberg 1981 ; d) K. Musigmann, Dissertation, Medizinische Universität zu Lübeck 1990.

[19] L. Schmerling, J. Am. Chem. Soc. 67 (1945) 1152.

[20] Dieser Vorschlag entspricht einem ,enforced mechanism“: W. P. Jencks, Acc. Chem. Res. 13 (1980) 161.

[21] R. Schneider, Dissertation, Universität Erlangen-Nürnberg 1987

[22] E. Bäuml, G. Kolberg, H. Mayr, Tetrahedron Lett. 28 (1987) 387.

[23] R. Pock, H. Mayr, Chem. Ber. 119 (1986) 2497.

[24] C. Schade, H. Mayr, unveröffentlichte Ergebnisse.

[25] R. Pock, H. Klein, H. Mayr, Chem. Ber. 119 (1986) 929.

[26] A. Streitwieser, Jr., C. H. Heathcock: Introduction to Organic Chemistry, 3. Aufl., Macmillan, New York 1985, S. 1153.

[27] C. Schade, H. Mayr, E. M. Arnett, J. Am. Chem. Soc. 110 (1988) 567

[28] S. W. Benson, Thermochemical Kinetics, 2. Aufl., Wiley-Interscience, New York 1976.

[29] Tatsächlich wurde für diese Reaktion das Salz $\left(\mathrm{ClC}_{6} \mathrm{H}_{4}\right)_{3} \mathrm{C}^{\oplus} \mathrm{BCl}_{4}^{\ominus}$ eingesetzt (Fall d), so da $\Delta G_{\mathrm{a}}^{\circ}$ hier sogar stark negativ sein muß: C. Schade, $\mathrm{H}$. Mayr, Makromol. Chem. Rapid Commun. 9 (1988) 477.

[30] H. Mayr, C. Schade, M. Rubow, R. Schneider, Angew. Chem. 99 (1987) 1059; Angew. Chem. Int. Ed. Engl. 26 (1987) 1029.

[31] Durch Kombination der Gleichungen 5 und 7 in : C. Schade, H. Mayr, Tetrahedron 44 (1988) 5761

[32] a) C. A. Grob, P. W. Schiess, Angew. Chem. 79 (1967) 1; Angew. Chem. Int. Ed. Engl. 6 (1967) 1; b) C. A. Grob, ibid. 81 (1969) 543 bzw. 8 (1969) 535; c) K. G. Becker, C. A. Grob in S. Patai (Hrsg.): The Chemistry of DoubleBonded Functional Groups, Suppl. A, Part 2, Wiley, London 1977, Kap. 8.

[33] Diese Grenze verschiebt sich natürlich, wenn das kovalente Produkt einen stabilen Komplex mit der Lewis-Säure bildet.

[34] K. Conrow, J. Am. Chem. Soc. 81 (1959) 5461.

[35] E. Bäuml, C. Schell, H. Mayr, unveröffentlichte Ergebnisse

[36] a) H. Mayr, R. Pock, Tetrahedron Lett. 24 (1983) 2155; b) H. Mayr, R. Pock, Chem. Ber. 119 (1986) 2473.
[37] H. Mayr, R. Schneider, Makromol. Chem. Rapid Commun. 5 (1984) 43.

[38] L. M. Dorfman, R. J. Sujdak, B. Bockrath, Acc. Chem. Res. 9 (1976) 352

[39] Y. Wang, L. M. Dorfman, Macromolecules 13 (1980) 63.

[40] R. Schneider, H. Mayr, P. H. Plesch, Ber. Bunsenges. Phys. Chem. 91 (1987) 1369 zit. Lit.

[41] R. Schneider, U. Grabis, H. Mayr, Angew. Chem. 98 (1986) 94; Angew Chem. Int. Ed. Engl. 25 (1986) 89.

[42] J. Smid in M. Szwarc (Hrsg.): Ions and Ion Pairs in Organic Reactions, Vol. 1, Wiley-Interscience, New York 1972, Kap. 3.

[43] a) H. Hostalka, G. V. Schulz, Z. Phys. Chem. Neue Folge 45 (1965) 286 b) D. N. Bhattacharyya, C. L. Lee, J. Smid, M. Szwarc, J. Phys. Chem. 69 (1965) 612; c) M. Szwarc in M. Szwarc (Hrsg.): Ions and Ion Pairs in Organic Reactions, Vol. 2, Wiley-Interscience, New York 1974 Kap. 4.

[44] Eine detailliertere Analyse der unterschiedlichen Situation bei Carbokationen und Carbanionen findet sich in: H. Mayr, R. Schneider, C. Schade Macromol. Chem. Macromol. Symp. 13/14 (1988) 43.

[45] C. Reichardt: Solvents and Solvent Effects in Organic Chemistry, 2. Aufl., VCH Verlagsgesellschaft, Weinheim 1988, S. 365.

[46] H. Mayr, R. Schneider, U. Grabis, J. Am. Chem. Soc. 112 (1990) 4460.

[47] Auch die Solvolysegeschwindigkeiten Methoxy-substituierter Diphenylmethylderivate sind aus diesem Grund schlecht mit $\sum \sigma^{+}$korreliert: S Nishida, J. Org. Chem. 32 (1967) 2692, 2695, 2697.

[48] a) D. Y. Curtin, Rec. Chem. Progr. 15 (1954) 111; b) L. P. Hammett Physical Organic Chemistry, McGraw-Hill, New York 1970; c) J. I. Seeman, Chem. Rev. 83 (1983) 83.

[49] J. P. Dau-Schmidt, H. Mayr, unveröffentlichte Ergebnisse.

[50] E. M. Arnett, C. Petro, P. von R. Schleyer, J. Am. Chem. Soc. 101 (1979) 522.

[51] O. Exner: Correlation Analysis of Chemical Data, Plenum Press, New York 1988, Kap. 2.4, zit. Lit.

[52] a) W. M. Schubert, J. R. Keeffe, J. Am. Chem. Soc. 94 (1972) 559; b) A. D. Allen, M. Rosenbaum, N. O. L. Seto, T. T. Tidwell, J. Org. Chem. 47(1982) 4234.

[53] J. H. Rolston, K. Yates, J. Am. Chem. Soc. 91 (1969) 1483.

[54] G. Collin, U. Jahnke, G. Just, G. Lorenz, W. Pritzkow, M. Röllig. L. Winguth, P. Dietrich, C.-E. Doering, H. G. Hauthal, A. Wiedenhöft, $J$. Prakt. Chem. 311 (1969) 238.

[55] A. Streitwieser, Jr.: Solvolytic Displacement Reactions, McGraw Hill, New York 1962, S. 43.

[56] Literaturübersicht siehe Tabelle 3 in [23].

[57] M. F. Ruasse, J. E. Dubois, A. Argile, J. Org. Chem. 44 (1979) 1173.

[58] a) W. K. Chwang, V. J. Nowlan, T. T. Tidwell, J. Am. Chem. Soc. 99 (1977) 7233; b) P. Knittel, T. T. Tidwell, ibid. 99 (1977) 3408; c) W. K. Chwang, P. Knittel, K. M. Koshy, T. T. Tidwell, ibid. 99 (1977) 3395.

[59] a) C. D. Ritchie, Acc. Chem. Res. 5 (1972) 348; b) C. D. Ritchie, Can. J. Chem. 64 (1986) 2239.

[60] a) A. Pross, J. Am. Chem. Soc. 98 (1976) 776; b) C. D. Ritchie, Pure Appl. Chem. 51 (1979) 153

[61] a) N. C. Deno, J. Jaruzelski, A. Schriesheim, J. Am. Chem. Soc. 77 (1955) 3044; b) N. C. Deno, A. Schriesheim, ibid. 77 (1955) 3051.

[62] Übersichten über SET-Processe: a) A. Pross, Acc. Chem. Res. $18(1985$ 212; b) L. Eberson, F. Radner, ibid. 20 (1987) 53; c) L. Eberson, Adv. Phys. Org. Chem. 18 (1982) 79; d) J. K. Kochi, Angew. Chem. 100 (1988) 1331 Angew. Chem. Int. Ed. Engl. 27 (1988) 1227.

[63] F. Freeman, Chem. Rev. 75 (1975) 439.

[64] a) Ionisationspotentiale aus der Datensammlung in [63]; sofern dort nich vorhanden aus: b) D. W. Turner, Adv. Phys. Org. Chem. 4 (1966) 31; c) Allyltrimethylsilan: A. Schweig, U. Weidner, G. Manuel, J. Organomet. Chem. 67 (1974) C4.

[65] B. Irrgang, H. Mayr, unveröffentlichte Ergebnisse.

[66] a) J. Bartl, S. Steenken, H. Mayr, R. A. McClelland, J. Am. Chem. Soc. 112 (1990) 6918; b) R. A. McClelland, N. Banait, S. Steenken, ibid. 108 (1986) 7023

[67] H. Mayr, G. Hagen, J. Chem. Soc. Chem. Commun. 1989, 91.

[68] J. Bartl, G. Hagen, B. Irrgang, H. Mayr, 22. GDCh-Hauptversammlung Bonn 1989, Poster OC46. 